\title{
ON CERTAIN NUMERICAL INVARIANTS OF ALGEBRAIC VARIETIES WITH APPLICATION TO ABELIAN VARIETIES*
}

\author{
BY \\ BOLOMON LEFSCHETZ \\ CONTENTS
}

Introduction.

Number

Part I. Preuminary Theory.

Chapter I. Cycles and integrals of algebraic varieties.

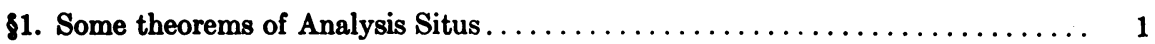

2. Effective and algebraic cycles. $\ldots \ldots \ldots \ldots \ldots \ldots \ldots \ldots \ldots \ldots \ldots \ldots \ldots$

3. Integrals of the second kind $\ldots \ldots \ldots \ldots \ldots \ldots \ldots \ldots \ldots \ldots \ldots \ldots \ldots \ldots$

4. Periods of integrals of the first kind $\ldots \ldots \ldots \ldots \ldots \ldots \ldots \ldots \ldots \ldots \ldots$

Chapter II. Invariants $\rho$ and $\sigma$ of algebraic varieties.

81. Poincare's normal functions for algebraic surfaces $\ldots \ldots \ldots \ldots \ldots \ldots \ldots \ldots$

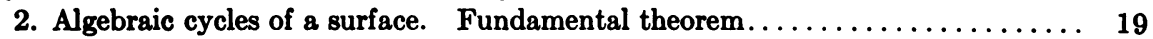

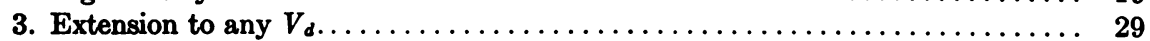

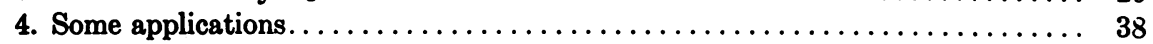

5. Varieties representing the groups of points of other varieties.......... 44

Part II. Abelian Varieties.

Chapter I. General properties.

81. A summary of some fundamental theorems and definitions . . . . . . . . 46

2. Connectivity of varieties of rank one $\ldots \ldots \ldots \ldots \ldots \ldots \ldots \ldots \ldots \ldots \ldots \ldots$

3. Intermediary functions $\ldots \ldots \ldots \ldots \ldots \ldots \ldots \ldots \ldots \ldots \ldots \ldots \ldots \ldots \ldots \ldots$

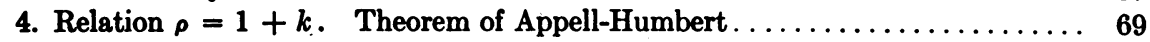

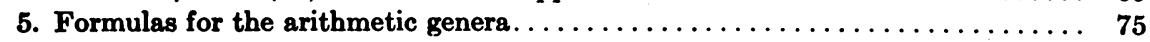

Chapter II. Abelian varieties with complex multiplication.

81. Generalities . . . . . . . . . .

2. Complex multiplication with irreducible characteristic equation $\ldots \ldots \ldots \ldots \ldots$

3. The characteristic equation is irreducible and Abelian $\ldots \ldots \ldots \ldots \ldots \ldots \ldots . . \ldots 0$

4. Characteristic equation of type $[f(\alpha)] r=0, r>1$. (a) Generalities...... 95

5. Characteristic equation of type $[f(\alpha)]^{r}=0$. (b) Two important special cases 102

6. Pure matrices of genus two or three $\ldots \ldots \ldots \ldots \ldots \ldots \ldots \ldots \ldots \ldots \ldots \ldots$

Chapter III. Abelian varieties with cyclic group and varieties of rank $>1$; images of their involutions.

81. Varieties of rank one with cyclic group $\ldots \ldots \ldots \ldots \ldots \ldots \ldots \ldots \ldots \ldots$

2. Multiple points of Abelian varieties of rank $>1 \ldots \ldots \ldots \ldots \ldots \ldots \ldots \ldots \ldots$

3. Connectivity of varieties of rank $>1 \ldots \ldots \ldots \ldots \ldots \ldots \ldots \ldots \ldots \ldots \ldots \ldots$

4. Integrals of the first kind. Invariants $[\rho], \rho, \rho_{0}^{2} \ldots \ldots \ldots \ldots \ldots \ldots \ldots \ldots$

- Presented to the Society November 27, 1920. This is a translation with minor modifcations of the memoir awarded the Bordin Prize by the Paris Academy of Sciences for the year 1919. The publication has been assisted by grants from the American Association for the Advancement of Science and the University of Kansas. 
Chapter IV. A class of algebraic curves with cyclic group and their Jacobi varieties.

81. Integrals of the first kind of the curves $y^{e}=\Pi_{i=1}^{r+2}\left(x-a_{i}\right) a_{i}$ ( $q$ odd prime) $\ldots 131$

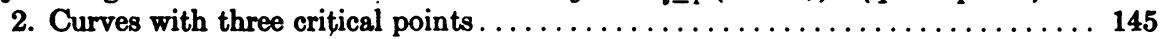

3. Curves with four critical points $\ldots \ldots \ldots \ldots \ldots \ldots \ldots \ldots \ldots \ldots \ldots \ldots \ldots \ldots \ldots$

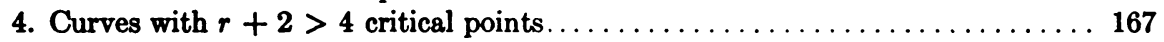

\section{Introduction}

In the development of the theory of algebraic functions of one variable the introduction by Riemann of the surfaces that bear his name has played a well-known part. Owing to the partial failure of space intuition with the increase in dimensionality, the introduction of similar ideas into the field of algebraic functions of several variables has been of necessity slow. It was first done by Emile Picard, whose work along this line will remain a classic. A little later came the capital writings of Poincare in which he laid down the foundations of Analysis Situs, thus providing the needed tools to obviate the failure of space intuition.

Meanwhile other phases of the theory were investigated in Italy and to some extent also in France, receiving an especially powerful impetus at the hands of Castelnuovo, Enriques and Severi. It is however a rather remarkable fact that in their work topological considerations are all but absent, practically never going beyond the study of linear cycles, and this on the whole by very indirect methods. The reason is to be found of course in the fact that the investigations of the Italian School all center around linear systems and the problems that they naturally suggest.

It seems certain that a further use of topological considerations will bring many new results. ${ }^{*}$ In such a development the notion of algebraic cycles, or cycles homologous to those formed by algebraic varieties, is destined to play an important part. It is in fact the central concept dominating the first half of this memoir.

After some general theorems on the analysis situs of algebraic varieties and on their multiple integrals, we concentrate our attention on surfaces, where, as could be expected, the results are most expressive. It is found first that a double integral of the first kind has no periods relatively to algebraic cycles, that is, to cycles homologous to those formed by algebraic curves. This result is then inverted by means of certain functions introduced by Poincaré. It follows that Picard's number $\rho$ is equal to the number of two-cycles without periods for the integrals of the first kind, while Severi's number $\sigma$ is the number of cycles whose multiple is homologous to zero. For higher varieties the result obtained is less precise, $\rho$ being merely at most equal to the number of

* In a monograph entitled L'Analysis Situs et la Géométrie Algébrique in course of publication in the Borel Series (Paris, Gauthiers-Villars), I have undertaken a thorough and systematic study of the topology of algebraic surfaces and varieties. Many new and interesting results, discovered since this memoir was written, will be found there. (Added in 1922). 
cycles without periods for the double integrals of the first kind. These general theorems are followed by various applications to the determination of $\rho$ and $\sigma$ in many interesting cases. We may mention in particular what seems to be the first complete proof of a theorem first considered by Noether, to the effect that an arbitrary algebraic surface of order greater than three in ordinary space, possesses only curves that are complete intersections.

The second and main part of this work is entirely devoted to Abelian varieties. Let $V_{p}$ be such a variety, of rank one and genus $p$,

$$
\Omega \equiv\left\|\omega_{j \mu}\right\| \quad(j=1,2, \cdot \cdots, p ; \mu=1,2, \cdots, 2 p)
$$

its period matrix. There exist in general $1+k$ linearly independent alternate forms with rational coefficients,

$$
\sum c_{\mu \nu} x_{\mu} y_{\nu}
$$$$
\left(c_{\mu \nu}=-c_{\nu \mu}\right),
$$

which vanish when the $(x)$ 's and the $(y)$ 's are replaced by the elements of any two rows of $\Omega$. In particular if a certain inequality due to Riemann is also satisfied, (1) is said to be a principal form of $\Omega$. Instead of the forms (1) we may consider others analogous to them, but no longer constrained to being alternate. The number of linearly independent forms of this more general type will be denoted by $1+h$ and we always have $h \geqq k \geqq 0$. The properties of matrices such as $\Omega$ have recently been the object of searching investigations by Scorza and Rosati.

Now it is found at once that $V_{p}$ possesses exactly $1+k$ two-cycles without periods with respect to the integrals of the first kind, and hence for $V_{p}$, $\rho \leqq 1+k$. On the other hand let $\phi\left(u_{1}, u_{2}, \cdots, u_{p}\right)$ be an intermediary function

$$
\phi\left(u+\omega_{\mu}\right)=e^{-2 \pi i\left(\sum_{(j)} a_{j \mu} u_{j}+\beta_{j}\right)} \cdot \phi(u)
$$

the relations which express its behavior relatively to $\Omega$. By considering the effect of adding the $\left(\omega_{\mu}\right)$ 's followed by the $\left(\omega_{\nu}\right)$ 's and then reversing the order we obtain at once $\sum_{(j)}\left(\alpha_{j \mu} \omega_{j_{\nu}}-\alpha_{j_{\nu}} \omega_{j_{\mu}}\right)=m_{\mu \nu}=-m_{\nu \mu}$ the $(m)$ 's being integers. We thus have corresponding to $\phi$ a certain alternate form with integral coefficients

$$
\sum m_{\mu \nu} x_{\mu} y_{\nu}
$$

On the other hand $\phi=0$ determines a certain algebraic hypersurface of $V_{p}$, and the intersection of $p-1$ hypersurfaces of the same system is a certain algebraic curve. By evaluating the cycle formed by this curve in terms of a certain simple fundamental system of cycles and then applying Riemann's inequality to the curve, we succeed in showing that the inverse of (2) is a certain principal form of $\Omega$ whence follows readily enough $\rho \equiv 1+k$, 
and therefore finally $\rho=1+k$. Thus the result obtained in the first part for algebraic surfaces holds for Abelian varieties. All this is to be compared with certain investigations of Appell, Humbert, Bagnera and de Franchis, leading in a wholly different manner to the same conclusion for $p=2$. There are also some related memoirs by Poincaré.

The consideration of Abelian varieties possessing certain complex multiplications leads us to the determination of $h, k, \rho$ in a wide range of cases. We touch here in many points investigations of Scorza, Rosati, and Frobenius, our methods being more nearly related to those of the last-named author. In the simplest type examined

$$
\Omega \equiv\left\|1, \alpha_{j}, \alpha_{j}^{2}, \cdots, \alpha_{j}^{2 p-1}\right\| \quad(j=1,2, \cdots, p),
$$

where the $(\alpha)$ 's together with their conjugates form the roots of an irreducible equation of degree $2 p$ with integer coefficients. The determination of $h, k, \rho$, is shown to be closely related to certain properties of the group of the equation. In particular if this group is Abelian it is shown that in general

$$
1+h=2(1+k)=2 \rho=2 p,
$$

and $V_{p}$ does not contain any Abelian subvariety of genus $<p$.

After considering some new properties of Abelian varieties of rank $>1$, we pass to the study of the curves

$$
y^{q}=\prod_{(i)}\left(x-a_{i}\right)^{\prime} \quad \text { ( } q \text { odd prime) }
$$

and of their Jacobi varieties with which the memoir terminates. We have succeeded in determining the total group of birational transformations when the (a)'s are arbitrary. In particular we have showed that in some welldefined cases there are no systems of reducible integrals, that the group is of order $q$ and that the invariants of the Jacobi variety are given by

$$
1+h=2(1+k)=2 \rho=2(q-1) .
$$

A remarkable class of curves met with occurs when $q-1$ is divisible by three. They are birationally transformable to the form

$$
x^{m} y^{n}+y^{m} z^{n}+z^{m} x^{n}=0 \quad\left(m^{2}-m n+n^{2}=q\right) .
$$

Their group is a collineation group of order $3 q$ if $q>7$. For $q=7$, we deal with the famous Klein quartic $x^{3} y+y^{3} z+z^{3} x=0$, whose group as shown by Klein is the classical simple collineation group of order 168 . In all cases we have for the Jacobi varieties $1+h=2(1+k)=2 \rho=6 p=3(q-1)$, and there are systems of reducible integrals of genus $(1 / 3) p$. Special cases have been considered by various authors (Klein, Ciani, Snyder, Scorza). 


\section{PART I \\ PRELIMINARY THEORY}

\section{Chapter 1. Cycles and integrals of algebraic varieties}

\section{$\S 1$. Some theorems of analysis situs}

1. We collect here some notations to be used frequently in the sequence. $V_{d}$, fundamental $d$-dimensional variety;

$S_{d}, d$-space;

$\left\{C_{u}\right\}$, linear pencil depending upon the parameter $u$ and generic in a linear system $|C|$ of hypersurfaces of $V_{d}$;

$R_{i}, i$ th connectivity index of $V_{d}$;

$M N \cdots P$, intersection of the varieties $M, N, \cdots, P$.

[ $M$ ] , arithmetic genus of $M$, the number of points of the group when $M$ is a group of points.

In general we shall denote indifferently by $M$ an algebraic variety or its Riemann-image. At times we shall find it convenient to distinguish between them; and then we shall denote the image by $(M)$. Finally by hypersurface of $V_{d}$ is to be understood a $(d-1)$-dimensional algebraic variety contained in it.

2. Let $V_{d}$ be an algebraic variety with ordinary singularities, $|C|$ an irreducible linear system, $\infty^{d}$ at least, without base points, contained in $V_{d}$, and such that no point of $V_{d}$ is multiple for any $C$ through it. Taking then a pencil $\left\{C_{u}\right\}$ we mark in the complex $u$ plane the critical points $a_{1}, a_{2}, \cdots, a_{n}$, or points where $C_{u}$ acquires a conical node and trace cuts $a_{0} a_{i}$. For the sake of simplification we shall denote the hypersurfaces $C_{a_{0}}, C_{a_{4}}$ by $C_{0}, C_{i}$. We propose to prove the following three propositions:

(a) Any $i$-cycle of $V_{d}(i<d)$ is homologous to a cycle wholly within a $C$, and when $i<d-1$, the two cycles bound at the same time in their respective manifolds.

(b) Any $d$-cycle is the sum of two others of which one is wholly within a $C$ and the other is composed of a $d$-dimensional manifold contained in $C_{0}$ plus the loci of certain $(d-1)$-cycles of $C_{u}$ when $u$ describes the lines $a_{0} a_{i}$.

(c) The $i$ th connectivity indices, $i \leqq d-2$, of $V_{d}$ and $C$ are equal.

For linear cycles $(a)$ and $(c)$ have already been proved by Picard, Castelnuovo, and Enriques, for two-cycles of algebraic surfaces by Poincaré, whose method adapted to the general case shall be used here.* We shall also apply his notations $\equiv, \sim$, and say with him: " $A$ is homologous to $B$ (or $A \sim B$ ), modulo $M$ ", to indicate that $A-B$ bounds in $M$.

*Journal de mathématiques pures et appliquées, ser. 6, vol.2 (1906), p. 157. See also the writer's paper in the Annals of Mathematics, vol. 21 (1920) as well as the monograph already referred to. 
3. Since the propositions to be proved are true for $d=2$ we can assume that they hold for a $V_{d-1}$ and prove them for a $V_{d}$. Any $i$-cycle of $C_{u}(i \leqq d-2)$ is homologous to a cycle contained in the base manifold $B$ of $\left\{C_{u}\right\}$. The deformation of the cycle in $B$ must be continuous when $u$ varies, hence it remains homologous to itself, which shows that any cycle of less than $(d-1)$ dimensions of $C_{u}$ is invariant when $u$ varies. Even if the cycle has more than $(d-2)$ dimensions this still applies when it is homologous to a cycle in $B$.

Let then $K_{u}$ be the $(2 d-1)$-dimensional locus of $C_{u}$ when $u$ describes the semi-straight line: argument $\left(u-a_{0}\right)=$ Const. The manifold $K_{u}$ cuts an $i$-cycle $\Gamma_{i}(i \leqq d)$ in a certain $(i-1)$-dimensional manifold. By considering the homeomorphism which exists between $C_{u}$ and $C_{0}$ when the above semi-straight line does not contain any critical point, we may show that the $(i-1)$-cycle just considered is homologous to a cycle in $C_{0}$. Hence by a slight change in Poincarés reasoning (loc. cit.) we can show that the cycle $\Gamma_{i}$ is homologous to a sum of cycles situated in the hypersurfaces $C_{h}$ plus a cycle $\Gamma_{i}^{\prime}$ obtained as follows: a certain cycle $\gamma_{i-1}^{h}$ of $C_{u}$, ( $u$ is assumed on $a_{0} a_{h}$ ), reduced to a point for $u=a_{h}$, has for locus when $u$ describes the line $a_{0} a_{h}$ a manifold $\Delta_{i}^{h}$ whose boundary is the position of $\gamma_{i-1}^{\prime \prime}$ in $C_{0}$. The cycle $\Gamma_{i}^{\prime}$ is the sum of $\sum \lambda_{h} \Delta_{i}^{h}$ and a manifold $\Delta_{i}$ sitúated wholly in $C_{0}$. According to a remark made above no cycle of less than $(d-1)$ dimensions of $C_{u}$ disappears at the critical points, hence if $i\left\langle d, \Gamma_{i}\right.$ is homologous to a sum of cycles situated in several $\left(C_{u}\right)$ 's. But when $C_{u}$ is displaced it can neither gain nor lose cycles of $V_{d}$. Hence the cycles situated in several $\left(C_{u}\right)$ 's are all homologous to cycles within one of them, for example $C_{0}$. This completes the proof for the first part of $(a)$.

In the case of a $d$-cycle $\Gamma_{d}$, besides the cycle in $C_{0}$ there is the cycle $\sum \lambda_{h} \Delta_{l}^{h}+\Delta_{d}$ which proves $(b)$. Let now $\Gamma_{i} \sim 0(i \leqq d-2)$ and denote by $M_{i+1}$ a manifold bounded by $\Gamma_{i}$. By considering the intersections of $M_{i+1}$ and $\Gamma_{i}$ with $K_{u}$ we can show that there is a manifold $M_{i+1}^{\prime}$, bounded by the cycle $\Gamma_{i+1}^{\prime}$ of $C_{0}$, to which $\Gamma_{i}^{\prime}$ has been reduced. The manifold $M_{i+1}^{\prime}$ will be the sum of a manifold in $C_{0}$ and of another locus of certain $i$-cycles of $C_{u}$ when $u$ describes the cuts. Just as before, since no cycles of less than $(d-1)$ dimensions disappear at the critical points, the part of $M_{i+1}^{\prime}$ exterior to $C_{0}$ can be suppressed. The cycle $\Gamma_{i+1}^{\prime}$ will therefore form the boundary for a manifold wholly in $C_{0}$. This completes the proof of $(a)$.

4. Let $\Gamma_{i-2}(i \leqq d+1)$ be an invariant $(i-2)$-cycle of $C_{u}$. It will be an arbitrary cycle of $C_{u}$ for $i<d+1$. Associated with the complex $u$ plane it will generate an $i$-dimensional manifold $\Gamma_{i}$ bounded by the locus of $\Gamma_{i-2}$ when $u$ describes the cuts. But the parts of this boundary generated by $\Gamma_{i-2}$ when $u$ describes the opposite borders of the same cut destroy each other, hence $\Gamma_{i}$ is an $i$-cycle. It is besides evident that if $\Gamma_{i} \sim 0$, also $\Gamma_{i-2} \sim 0 \bmod$. 
$C_{u}$. Conversely, if $\Gamma_{i-2}$ bounds an $(i-1)$-dimensional manifold on $C_{u}$, $\Gamma_{i}$ bounds its $(i+1)$-dimensional locus when $u$ describes the complex $u$ plane. There might be doubt only if $i-2=d-2$, for then parts of the locus corresponding to the cuts might also have to be considered as forming part of the boundary of the $(i+1)$-dimensional manifold. But in that case we replace $\Gamma_{d-2}$ by a homologous cycle in $C C_{u}$, ( $C$ fixed). This amounts to replacing $V_{d}$ by $C$ for which the difficulty does not present itself any more. A certain cycle $\sim \Gamma_{i}$ will then bound in $C$ and therefore also in $V_{d}$. In this manner $R_{d-1}$ independent cycles of $V_{d}$ in $C_{u}$ generate $R_{d-1}$ independent $(d+1)$ cycles of $V_{d}$, and as $R_{d-1}=R_{d+1}$, (Poincaré, loc. cit.), every $(d+1)$-cycle depends upon those so obtained. Let us start then with a $\Gamma_{d-i}$ of $V_{d}$ in a $C^{i}$. It yields first a $\Gamma_{d-i+2}$ in a $C^{i-1}$, which in its turn yields a $\Gamma_{d-i+4}$ in a $C^{i-2}$ etc., so that ultimately we arrive at a $\Gamma_{d+i}$ of $V_{d}$ of which $\Gamma_{d-i}$ is the trace on $C^{i}$, and if a multiple of one of the cycles is $\sim 0$, then also a multiple of the other is $\sim 0$. Owing to Poincaré's relation $R_{d-i}=R_{d+i}$, every $(d+i)$-cycle depends upon those thus obtained or conversely all $(d-i)$-cycles of $V_{d}$ in $C^{i}$ are dependent upon the traces of its $(d+i)$-cycles. Now a $(d-i)$ cycle in $C^{i}$ can just as well be considered as the trace of a $(d+i-2)$-cycle in a $C$ passing through $C^{i}$, and all $(d-i)$-cycles of $C^{i}$ depend upon the traces of these $(d+i-2)$-cycles of $C$. The reverse is true, namely, all the $(d+i-2)$-cycles of $C$ depend upon the cycles generated by the $(d-i)$ cycles in the ( $\left.C^{i}\right)$ 's. It follows that the index $R_{d+i-2}$ of $C$ is equal to $R_{d+i}$, or, owing to Poincaré's relations, as applied to $C$, the indices $R_{d-i}$ of both manifolds are equal. This proves $(c)$.

\section{$\S 2$. Effective and algebraic cycles}

5. It is important to distinguish between the cycles which do and those which do not intersect a given hypersurface $C$. If we merely limit our considerations to an assigned cycle or hypersurface we can not go very far, for the one can be deformed and the other displaced in its continuous system, their intersection thereby being changed. To arrive at a valuable concept we must compare a continuous system of hypersurfaces to a set of homologous cycles. We shall say by definition that an $i$-cycle $\Gamma_{i}$ is effective relatively to the hypersurface $C$ if there is a homologous cycle having no point of intersection with a hypersurface of the same continuous system as $C$.

Let then $|C|$ be a linear system such as we have already considered, and assume that a cycle $\Gamma_{i}(i \leqq d)$ meets a $C$ along a $\Gamma_{i-2}$.

In the intersection $C C_{1}$ of $C$ with another hypersurface $C_{1}$ of the system there exists, (No. 2), a $\Gamma_{i-2}^{\prime}$ trace of a cycle $\Gamma_{i}^{\prime \prime}$ of $C, \sim \bmod . C$ to a multiple of $\Gamma_{i-2}$. Replacing if necessary $\Gamma_{i}$ by an adequate multiple, we may say that $\Gamma_{i-2}^{\prime} \sim \Gamma_{i-2}$ mod. $C$. Now make $C_{1}$ tend towards $C$ in $|C|$, while main- 
taining $\left|C C_{1}\right|$ and $\Gamma_{i-2}^{\prime}$ fixed. $\Gamma_{i}^{\prime \prime}$ will tend towards a cycie $\Gamma_{i}^{\prime}$ of $C_{1}$, whose trace on $C C_{1}$ will still be $\Gamma_{i-2}^{\prime}$. We thus have a cycle $\Gamma_{i}^{\prime}$ of $C_{1}$, whose trace on $C C_{1}$ bounds with $\Gamma_{i-2}$ a certain $M_{i-1}$ of $C$.

Let now $E_{2}$ be a two-dimensional elementary sensed manifold or "element" passing through a point $A$ of $C$ and transverse to $C$. Together with a sensed (2d-2)-fold element of $C$ through $A$ it determines the sensing of $V_{d}$, and since $C$ and $V_{d}$ are both two sided, when $A$ describes any closed path in $C, E_{z}$ may be displaced at the same time without ever acquiring a line segment in common with $C$, so as to return to its former position with sense unchanged. The following analogous occurrence in ordinary space will make this clearer: When the origin $A$ of a directed segment $A B$ describes a closed path on a twosided surface, $A B$ may be displaced without ever becoming tangent to the surface, so as to return exactly to its original position.

To come back to our question, let $A$ describe $M_{i-1}$ and displace $E_{2}$ in the manner just indicated so that moreover when $A$ is in $\Gamma_{i-2}$ or $\Gamma_{i-2}^{\prime}, E_{2}$ will be in $\Gamma_{i}$ or $\Gamma_{1}^{\prime}$ respectively. In these conditions a circuit surrounding $A$ in $E_{2}$ will generate a manifold $M_{i}$ meeting nowhere $C$, and if we suppress in $\Gamma_{i}-\Gamma_{i}^{\prime}+M_{i}$ the part generated by the area of $E_{2}$ which is bounded by $\zeta$ when $A$ describes $\Gamma_{i-2}$ or $\Gamma_{i-2}^{\prime}$, we obtain a cycle $\sim\left(\Gamma_{i}-\Gamma_{i}^{\prime}\right)$, since we can make it tend towards this cycle as a limit, and this new $i$-cycle is effective relatively to $C$. But $\Gamma_{i}^{\prime}$, which is in $C_{1}$, is $\sim$ to a cycle wholly in $C$. Hence, there exists a combination of any $i$-cycle $(i \leqq d)$ and of cycles wholly in $C$, effective with respect to $C$.

Algebraic cycles. These cycles, which occur when $i$ is even, are simply the cycles homologous to a combination of those formed by the algebraic manifolds of $V_{d}$. The cycles formed by algebraic curves have been considered by Poincaré although he made no application of them.

6. A non-bounding $d$-cycle of $C$ is also one of $V_{d}$, for when $C$ varies in its system it neither gains nor loses $(d-2)$-cycles and therefore $d$-cycles, so that they are invariant. Hence the number of $d$-cycles non-effective relatively to $C$ is equal to the $d$ th index of connectivity of the hypersurface, hence also to its $(d-2)$ th index and therefore, according to what we have already shown, to $R_{d-2}$. If we denote by $R_{i}^{\prime}$ the number of $i$-cycles effective with respect to $C$, we shall have $R_{d}^{\prime}=R_{d}-R_{d-2}$.

Let now $i \leqq d-1$. Any $i$-cycle of $V_{d}$ is homologous to a cycle in $C^{d-i}$. Replacing then $V_{d}$ by $C^{d-i}$, which has the same number of effective $i$-cycles with respect to a $C^{d-i+1}$ as $V_{d}$ with respect to $C$, we obtain

$$
R_{i}-R_{i}^{\prime}=R_{i-2}, \quad R_{2}-R_{2}^{\prime}=1 \quad(2<i \leqq d) .
$$

The second formula follows from the fact that we have to take into account the non-effective algebraic cycle $C^{d-1}$. 
In the next chapter it will be shown that on an algebraic surface the algebraic curves are non-effective cycles with respect to a curve in a properly chosen system. Moreover, from the above it follows that if $C$ is a plane section, $A$ any other curve, $\Gamma$ any two-dimensional cycle, a certain cycle $m A+n \Gamma(n \neq 0)$ is effective with respect to $C$. The proof holds as well if $C$ is generic in a certain linear system $|C|$ analogous to the system of hypersurfaces of similar name. This establishes the second formula for $d=2$. When $d>2$, a multiple of any $\mathrm{F}_{2}$ is homologous to a cycle in $C$, which shows that we may replace $V_{d}$ by $V_{d-1}$; hence this second formula holds for all cases.

We thus see that $R_{i}^{\prime}$ does not depend upon the special system $|C|$ considered, provided it has certain general properties specified in No. 2.

7. From the formulas obtained follow these:

$$
\begin{aligned}
& R_{2 i}=\sum_{h=0}^{i} R_{2 h}^{\prime}, \quad 2 i \leqq d \\
& R_{2 i+1}=\sum_{h=0}^{i} R_{2 h+1}^{\prime} ; \quad 2 i+1 \leqq d ; \\
&\left(R_{1}^{\prime}=R_{1}, \quad R_{0}=1\right) .
\end{aligned}
$$

Hence if $R_{2 i}=1$, its minimum value, $R_{2 h}^{\prime}=0, R_{2 h}=1, h \leqq i$, and if $R_{2 i+1}=0, R_{2 h+1}^{\prime}=R_{2 h+1}=0, h \leqq i$. In particular if a single $R_{2 i+1}=0$, $R_{1}=0$ and $V_{d}$ is regular.

Finally let us notice explicitly that a multiple of an i-cycle non-effective with respect to $C$ is the sum of an effective cycle and of a cycle in $C^{d-i+1}$. For $i=2$, the multiple of any non-effective cycle is the sum of an effective cycle and the algebraic cycle $C^{d-1}$ taken a certain number of times.

Let us mention here a formula derived first by Alexander* and giving $R_{d}$ in terms of the $\left(R_{i}\right)$ 's and of the Zeuthen-Segre invariant $I_{d}$ of $V_{d}$. It can be written

$$
R_{d}=I_{d}+2 \sum_{i=1}^{d-1}(-1)^{d-i+1} R_{i}+2(-1)^{d}(d-1) .
$$

8. Let $A_{1}, \cdots, A_{\mathrm{q}}$ be hypersurfaces of $V_{d}$ and assume $|C|$ so chosen that it contains their sum, leaving a residue $|K|$, linear system of the same type as $|C|$. Let now $C_{0}=A_{1}+A_{2}+\cdots+A_{2}+K$, and, instead of considering a pencil $\left\{C_{u}\right\}$ generic in $|C|$, let us take one containing $C_{0}$. Reasoning in regard to this pencil as previously we find that the multiple of any $d$-cycle is the sum of a cycle effective with respect to the hypersurfaces $A_{r}, K$, and of a cycle contained within one or more of these hypersurfaces, and non-effective with respect to their totality. The number of effective cycles will now be $R_{d}^{\prime}-r_{d}, r_{d} \geqq 0$. Thus there will be $r_{d}$ cycles effective with respect to $K$,

\footnotetext{
* Rendiconti dei Lincei, ser. 5 vol. 23 (1914), pp. 55-62.
} 
but not with respect to the $(A)$ 's. Since $r_{d} \leqq R_{d}^{\prime}$, when any set of hypersurfaces whatever is considered, this integer has a maximum $\rho_{d}$ which is obviously a numerical invariant of $V_{d}$. It is the maximum number of cycles contained in the hypersurfaces of $V_{d}$ and yet effective with respect to an irreducible linear system, $\infty^{d}$ at least, without base points.

A similar invariant $\rho_{i}$ may be defined for $i$-cycles. The invariants $\rho_{i}$ of $V_{d}$ and of $C$ are the same when $i \leqq d-2$. Hence $\rho_{i}$ is the same for $V_{d}$ and $C^{k}, k \leqq d-i-1$. In particular $\rho_{2}$ is the same for $V_{d}$ and $C$. We shall see that for any $V_{d}, 1+\rho_{2}$ is equal to Picard's number $\rho$.

9. Torsion indices. It may happen that two $i$-cycles $\Gamma_{i}, \Gamma_{i}^{\prime}$, without being homologous to each other, are nevertheless such that $\lambda \Gamma_{i} \sim \lambda \Gamma_{i}^{\prime}, \lambda>1$. The cycle $\Delta_{i}=\Gamma_{i}-\Gamma_{i}^{\prime}$ is called a zero-divisor. Poincaré has shown that when this occurs, $V_{d}$ undergoes a sort of internal torsion and the $(\lambda)$ 's are related to his so-called torsion-coefficients. He further established that the torsion coefficients for $i$-cycles are equal to those for the $(2 d-i-1)$-cycles.

The operations $+\Delta_{i}$ applied to the $i$-cycles give rise to an abelian group whose order $\sigma_{i}$ is the product of the torsion coefficients. We shall call it the $i$ th torsion index. These indices satisfy the relation $\sigma_{i}=\sigma_{2 d-i-1}, \sigma_{2 d-1}=1$. In particular, $\sigma_{1}=\sigma_{2 d-2}=\sigma$ is the invariant of Severi, as we shall see later. That is in an algebraic surface it turns out that the zero divisors for the cycles or algebraic curves lead all to the same numerical invariant. Algebraic surfaces with $\sigma>1$, of which examples have been given by Severi and Godeaux, have then the property of possessing linear cycles that do not bound, but of which a certain multiple bounds.

Since any $i$-cycle, $i \leqq d-2$, is homologous to a cycle in $C$ and also since such cycles when in $C$ form cycles of $V_{d}, V_{d}$ and $C$ have the same zero divisors for these cycles and therefore equal invariants $\sigma_{i}, i \leqq d-2$.

10. The notions of base, intermediary base, minimum base are commonly useful. Let $A_{1}, A_{2}, \cdots$ be a set of entities forming a modulus, i.e., having these properties: (a) The sum or difference of any two belong to the set, $(b)$ the zero of the set is well defined, (c) $A+B$ does not differ from $B+A$. The entities $A_{1}, \cdots, A_{n}$ are said to form a base if for every $A$ there is a relation $\lambda A=\lambda_{1} A_{1}+\lambda_{2} A_{2}+\cdots+\lambda_{n} A_{n}$, where the ( $\lambda$ )'s are integers. If $\lambda$ always divides all the $\left(\lambda_{i}\right)$ 's the base is called intermediary, while if for every $A$ we can take $\lambda=1$, we have to deal with a minimum base. Examples of such systems are given by the $i$-cycles of a closed manifold, the hypersurfaces of a $V_{d}$, etc.

Repeating for the $i$-cycles a discussion of Severi's* for algebraic curves it can be shown that the $i$-cycles of $V_{d}$ possess ordinary and minimum bases composed of $R_{i}$ and $R_{i}+\sigma_{i}-1$ cycles respectively.

* Annales de l'Ecole Normale supérieure, ser. 2, vol. 25 (1908), pp. 449-468. The nomenclature is his. 
11. If $V_{d}$ possesses arbitrary singularities but is birationally transformable into $V_{d}^{\prime}$, variety with ordinary singularities, the neighborhood of the extraordinary singularities of $V_{d}$ may be considered as forming a certain number of infinitesimal algebraic varieties. We shall thus be led to consider infinitesimal $i$-cycles contained in these varieties and corresponding to finite cycles on $V_{d}$.

In general an invariant $m$ may take a different value according as infinitesimal cycles are or are not taken into consideration. Reserving the ordinary notation for the first case we shall denote the invariant in the second by $[m]$. This notation has already been used by Bagnera and de Franchis for the number $\rho$. We shall then have $R_{i}-\left[R_{i}\right]=\rho_{i}-\left[\rho_{i}\right]$.

Remark. If $V_{d}$ has ordinary singularities, in considering the $i$-cycles contained in a given hypersurface, the infinitesimal cycles must be left out, that is, no attention must be paid to accidental singularities of the hypersurface.

\section{$\S 3$. Integrals of the second kind}

12. Much interest is added to the discussion by introducing integrals of the second kind. The author has recently published a series of results (most of them without proofs) relating to triple integrals of a $V_{3}^{*}$ and a memoir containing the proofs is ready for publication. In another memoir he has given the extension to double integrals of a $V_{3} \dagger$, of the Picard theory of double integrals of the second kind of algebraic surfaces. The extension from $d=2$ to $d=\mathbf{8}$ presents many difficulties which seem to disappear in the extension from $d=3$ to any other value of $d$. We shall then indicate mostly without proofs the statements for a $V_{d}$ :

Let $F_{d}\left(x_{1}, x_{2}, \cdots, x_{d}, t\right)=0$ be the equation of $V_{d}$ still assumed irreducible and with ordinary singularities, $|H|$ the system of hyperplane sections, $H_{x_{i}}$ the section by $x_{i}=$ const. The integral

(1) $\iint \cdots \int U\left(x_{1}, x_{2}, \cdots, x_{d}, t\right) d x_{1} d x_{2} \cdots d x_{d}$ ( $U$ a rational function),

is of the second kind if to every hypersurface of infinity $A$ of $U$ corresponds an integral of type called improper of the second kind,

$$
\iint \cdots \int \sum \frac{\partial U_{i}}{\partial x_{i}} d x_{1} d x_{2} \cdots d x_{d} \quad\left(U_{i} \text { a rational function }\right),
$$

such that their difference is finite in the neighborhood of an arbitrary point of $A$. If (1) is not of the type (2) it is said to be proper of the second kind.

Proper and improper integrals preserve their character under birational

* Comptes Rendus, vol. 165 (1917), pp. 850-854.

† Annali di matematica, ser. 3, vol. 26 (1917), pp. 227-261. 
transformations. The number $\rho_{0}^{d}$ of proper integrals of which no linear combination is improper is an absolute invariant. If the system of hyperplane sections is adequately selected every integral of the second kind can be reduced by subtraction of an improper integral to the normal form

$$
\iint \cdots \int \frac{P\left(x_{1}, x_{2}, \cdots x_{d}, t\right)}{F_{t}^{\prime}} d x_{1} d x_{2} \cdots d x_{d},
$$

where $P$ is an adjoint polynomial.

Whether of the second kind or not, (3) has periods and residues, but only relatively to finite cycles or cycles effective relatively to the hypersurface at infinity. Indeed, as Picard has shown, if the cycle is infinite the corresponding value of (3) is indefinite or if definite may vary when the cycle is deformed.

In connection with (3) we may also consider

$$
\iint \cdots \int \frac{P\left(x_{1}, x_{2}, \cdots, x_{d}, t\right)}{F_{t}^{\prime}} d x_{1} d x_{2} \cdots d x_{d-1},
$$

attached to $H_{x_{d^{*}}}$ Its periods satisfy a linear homogeneous differential equation-the equation of Picard-Fuchs. By expressing the periods of (3) in terms of those of (4) we may show that when $P$ is properly chosen they assume an arbitrary value.

13. Let then $r_{i}^{\prime}$ be the number corresponding to $R_{i}^{\prime}$ for $H$. On the strength of what has been stated we may derive this formula, in which $N$ denotes the class of $V_{d}, R_{d}^{\prime}=N-2\left(r_{d-1}^{\prime}-R_{d-1}^{\prime}\right)-\left(r_{d-2}^{\prime}-R_{l-2}^{\prime}\right)$, as being the exact number of integrals without residues, but with some non-zero periods. These integrals are of the second kind and every proper integral is reducible to them.

By means of this relation we may derive Alexander's formula for $R_{d}$. Indeed, if $I_{s}$ is the Zeuthen-Segre invariant of $H^{d-s}$ we have for $V_{d}$ :

$$
\begin{aligned}
I_{d} & =N-2 I_{d-1}-I_{d-2} \\
\therefore \quad R_{d}^{\prime}-I_{d} & =2 R_{d-1}^{\prime}+R_{d-2}^{\prime}-2\left(r_{d-1}^{\prime}+I_{d-1}\right)-\left(r_{d-2}^{\prime}-I_{d-2}\right)
\end{aligned}
$$

and similarly for $H^{d-s}(s=3,4, \cdots, d-1)$,

$$
\begin{gathered}
r_{s}^{\prime}-I_{s}=2 R_{s-1}^{\prime}+R_{s-2}^{\prime}-2\left(r_{s-1}^{\prime}-I_{s-1}\right)-\left(r_{s-2}^{\prime}-I_{s-2}\right), \\
r_{2}^{\prime}-I_{2}=2 R_{1}+1,
\end{gathered}
$$

whence

$$
R_{d l}^{\prime}=I_{d}^{\prime}+(-1)^{d} \cdot(d-1)+\sum_{i=0}^{d-2}(-1)^{d-i} \cdot(d-i) \cdot R_{i+1}^{\prime},
$$

which reduces to Alexander's formula for $R_{d}$ if we remember that

$$
R_{i}^{\prime}=R_{i}-R_{i-2}
$$

and set, as we should, $R_{0}=1, R_{-1}=0$. 
Let us return to our integral of the second kind under normal form. If its periods are all zero, it is improper of the second kind. We shall show below that there are exactly $\rho_{d}$ improper integrals with non-zero periods, hence for the number of proper integrals

$$
\begin{aligned}
\rho_{0}^{\prime \prime} & =R_{d}^{\prime}-\rho_{d}=I_{d}+2(-1)^{d}(d-1)-\rho_{d}+\sum_{i=0}^{\prime l-2}(-1)^{d-i}\left(R_{i+1}-R_{i-1}\right) \\
& =I_{d}+2(-1)^{d}(d-1)-\rho_{d}-R_{d-2}+2 \sum_{i=1}^{d-1}(-1)^{d-i+1} R_{i} .
\end{aligned}
$$

For $d=2$ this becomes a well-known formula given by Picard and for $d=3$ it becomes the formula of the author's Comptes Rendus note, the $\lambda$ of the note being the same as $\rho_{d}$.

14. We will now outline the proof that there exist $\rho_{d}$ improper integrals in normal form and with non-zero periods.

Let then $A_{1}, A_{2}, \cdots, A_{n}$ be hypersurfaces such that there are $\rho_{d}$ finite cycles $\Gamma_{1}, \Gamma_{2}, \cdots, \Gamma_{\rho_{d}}$ non-effective relatively to some of the (A)'s. We shall assume (as we may) that the simple hypersurface of intersection of $F_{t}^{\prime}=0$ with $V_{d}$ is among the $(A)$ 's. There are $R_{t l}^{\prime}-\rho_{d}$ cycles, $\Gamma_{1}^{\prime}, \Gamma_{2}^{\prime}, \cdots, \Gamma_{R_{d}^{\prime}-\rho_{d}}^{\prime}$, effective relatively to $H$, or as a special case finite, which do not meet the $(A)$ 's.

It is sufficient to show that from any integral of the second kind in the normal form we may subtract an improper one so that the difference will be without periods relatively to the $(\Gamma)$ 's. Indeed, by means of a change of variables it can be shown that (4), extended to a closed continuum, where it is finite and where $F_{t}^{\prime} \neq 0$, yields a zero period.* It follows that an improper integral under the normal form has no period relatively to the $\left(\Gamma^{\prime}\right)$ 's and therefore the $R_{d}^{\prime}-\rho_{d}$ integrals that may be formed having no periods with respect to the ( $\Gamma$ )'s, but having some with respect to the $\left(\Gamma^{\prime}\right)$ 's, are certainly proper. As by assumption all proper integrals are reducible to them, we must have $\rho_{0}^{d}=R_{l}^{\prime}-\rho_{d}$. Therefore there must be $\rho_{d}=R_{d}^{\prime}-\rho_{0}^{d}$ improper integrals in the normal form with non-zero periods.

Let $\Gamma_{i k}$ be the $(d-2)$-cycle intersection of $\Gamma_{i}$ and $A_{k}$. A multiple of $\Gamma_{i k}$ is homologous mod. $A_{k}$ to a $\bar{\Gamma}_{i k}$ situated in $H_{x_{1}} A_{k}$, wherein it is invariant when $x_{1}$ varies. Replacing if need be $\Gamma_{i}$ by a certain multiple of itself we may assume that actually $\Gamma_{i k} \sim \bar{\Gamma}_{i k}$ mod. $A_{k}$. The possible exceptional singularities of $A_{k}$ need not be taken into account for the theorems of No. 2 are readily seen to hold even then since $\Gamma_{i k}$ is of dimensionality $d-2$ and $\Gamma_{i}$ may be assumed not to go through the exceptional singularities.

By reasoning as in No. 5 , we may reduce $\Gamma_{i}$ to a cycle cutting $A_{k}$ in $\bar{\Gamma}_{i k}$ and nowhere else. Henceforth assume that $\Gamma_{i}$ is that very cycle, $\Gamma_{i k}$ thus coinciding with $\bar{\Gamma}_{i k}$. We can then arrange matters so that the vicinity of $\Gamma_{i k}$

\footnotetext{
* The proof for $d=2$ is given in the author's A n a li di matematica paper, no. 8 .
} 
in $\Gamma_{i}$ be entirely in $H_{x_{1}}$. It constitutes a small region bound by a $(d-1)-$ cycle $\Delta_{i k}$. Suppress these regions from $\Gamma_{i}$ and let $M_{i}$ be what is left.

We observe that if $U_{1}$ is a rational function infinite on the $(A)$ 's only

$$
\begin{gathered}
\iint \cdots \int_{M_{i}} \frac{\partial U_{1}}{\partial x_{1}} d x_{1} d x_{2} \cdots d x_{d}=\sum \iint \cdots \int_{\Delta_{i k}} U_{1} d x_{2} d x_{3} \cdots d x_{d} \\
\iint \cdots \int_{M_{i}} \frac{\partial U}{\partial x_{h+1}} d x_{1} d x_{2} \cdots d x_{d}=0 .
\end{gathered}
$$

Thus the first $d$-uple integral is a sum of residues of

$$
\iint \cdots \int U_{1} d x_{2} d x_{3} \cdots d x_{d}
$$

attached to $H_{x_{1}}$, relatively to the cycles $\Gamma_{i k}$.

It may be shown that the residues at finite distance of $(5)^{*}$ are as arbitrary as if $U_{1}$ were no more constrained to be rational in $x_{1}$. This follows in principle from the fact that an integral of a rational function belonging to a $V_{d}$ and with arbitrary residues can be formed by operations rational with respect to the coefficients of its equation.

A corollary is the existence of an integral (5) with constant residues with respect to the cycles such as $\Gamma_{i k}$. In that case

$$
\frac{\partial}{\partial x_{1}} \iint \cdots \int U_{1} d x_{2} d x_{3} \cdots d x_{d}
$$

behaves like an integral of the second kind at finite distance. From this can be deduced the existence of rational functions $U_{2}, U_{3}, \cdots, U_{d}$, such that

$$
\sum_{i=1}^{d} \frac{\partial U_{i}}{\partial x_{i}}=\frac{P\left(x_{1}, x_{2}, \cdots, x_{d}, t\right)}{\phi\left(x_{1}\right) F_{t}^{\prime}}
$$

where $P$ is an adjoint polynomial and $\phi$ an ordinary polynomial. From the relations established above, and since the integral at the left in (7), taken over $\Gamma_{i}-M_{i}$, is very small, follows then

$$
\iint \cdots \int_{\mathrm{r}_{\mathrm{i}}} \frac{P \cdot d x_{1} d x_{2} \cdots d x_{d}}{\phi \cdot F_{\imath}^{\prime}}=\sum \iint \cdots \int_{\Delta_{i k}} U_{1} d x_{2} d x_{3} \cdots d x_{d} .
$$

Hence the period of (7) as to $\Gamma_{i}$, like the residues of (5) as to $\Gamma_{i k}$, is completely arbitrary. Thus we may obtain an improper integral of the second kind (7) with arbitrary periods relatively to the ( $\Gamma$ )'s. As (7) may be shown to be reducible to the normal form without gain or loss of periods, we see finally that its periods relatively to the $\left(\Gamma^{\prime}\right)$ 's are zero since these last cycles are effective relatively to the $(A)$ 's. As the periods of $(7)$ relatively

\footnotetext{
* $Q$ uarterly journal of mathematics, vol. 49 (1917), pp. 333-343.
} 
to the $(\Gamma)$ 's are perfectly arbitrary we may obtain an improper integral in the normal form which when subtracted from a given integral of the second kind in that form will yield one without periods with respect to the $(\Gamma)$ 's, and this proves our theorem.

All that precedes may be extended to integrals of "total differentials," that is, to integrals of the type $\mathcal{S} \mathcal{S} \cdots \mathcal{S} \sum P_{i_{1} i_{2} \ldots i_{1}} d x_{i_{1}} d x_{i_{2}} \cdots d x_{i_{\text {a }}}$, where the $(P)$ 's are rational and satisfy Poincaré's conditions of integrability. For example, when $s=2$, these conditions are

$$
\frac{\partial P_{i k}}{\partial x_{l}}+\frac{\partial P_{k l}}{\partial x_{i}}+\frac{\partial P_{l i}}{\partial x_{k}}=0 .
$$

The integral will be proper or improper of the second kind according as it determines an integral of one or the other type on an arbitrary $H$. This makes it possible to define these integrals by recurrence. The number of proper integrals of the second kind is $\rho_{0}^{s}=R_{s}^{\prime}-\rho_{s}=R_{s}-R_{s-2}-\rho_{s}$.

In particular for $s=2$ we have $\rho_{0}^{2}=R_{2}^{\prime}-\rho_{2}=R_{2}^{\prime}-(\rho-1)=R_{2}-\rho$. This special case $s=2$ has already been considered* by the author of the present paper.

\section{$\S 4$. Periods of integrals of the first kind}

15. Integrals of the first kind are defined as usual as those which are everywhere finite. While restrictions were necessary before as to cycles relatively to which integrals of the second kind could properly be assumed to have periods, they cease to be necessary when we deal with integrals of the first kind. However, the question may be asked, how many of the $R_{s}$ periods which an s-uple integral of the first kind may have, are linearly independent in the field of rational numbers, in the sense that they do not have to satisfy a linear homogeneous equation with integral coefficients. Let us assume at first $s=d$ and consider the integral of the first kind

$$
\iint \cdots \int \frac{P\left(x_{1}, x_{2}, \cdots, x_{d}, t\right)}{F_{t}^{\prime}} d x_{1} d x_{2} \cdots d x_{d} .
$$

What can be said as to its period relatively to a $d$-cycle $\Gamma_{d}$ non-effective relatively to $H$ ? By combining $\Gamma_{d}$ with effective cycles we may derive a cycle contained in $H_{x_{1}}$. Grant that this has already been done for $\Gamma_{d}$. It will intersect the $H^{2}$ at infinity of $H_{x_{1}}$ in a $(d-2)$-cycle $\Gamma_{d-2}$. We may isolate on $\Gamma_{d}$ the vicinity of $\Gamma_{d-2}$, the boundary of the isolated part $\Gamma_{d l}^{\prime}$ being a manifold $M_{d-1}-$ a tube for $d=3$. The integral (8) extended to $\Gamma_{d}-\Gamma_{d}^{\prime}$ gives zero since $d x_{1}=0$ at all points of this continuum and it is at finite distance. Let us make a projective transformation reducing the $H^{2}$ at in-

* Annali di matematica, loc. cit. In the formula there given $R_{2}$ should be replaced by $R_{2}^{\prime}$ as only finite cycles are considered. 
finity of $H_{x_{1}}$ to $H_{x_{1}} H_{x_{3}}$. Keeping notations unchanged we see that the period of (8) relatively to $\Gamma_{d}$ is equal to the value of (8) extended over $\Gamma_{d}^{\prime}$, a portion of the cycle near $\Gamma_{d-2}$, and this will certainly give zero if

$$
\iint \cdots \int \frac{P\left(x_{1}, x_{2}, \cdots, x_{d}, t\right)}{F_{t}^{\prime}} d x_{3} d x_{4} \cdots d x_{d}
$$

has no period relatively to $\Gamma_{d-2}$. Now if $\Gamma_{d-2}$ is finite, that is, if it is effective relatively to $H$, the period in question is certainly zero. For, maintaining $x_{1}$ fixed, we see that the periods of (9) vanish when $x_{2}=\infty$ because $P$ is an adjoint canonical polynomial (that is of degree $m-d-2$, where $m$ is the degree of $V_{d}$ ). But as $x_{2}$ varies the period relatively to $\Gamma_{d-2}$, cycle of $V_{d}$ in $H_{x_{1}} H_{x_{2}}$, is rational in $x_{2}$, finite everywhere since the cycle is finite, hence constant and zero at infinity, and therefore identically zero.

The same result can be obtained differently. The doubtful part of $\Gamma_{d}$ is composed of $\infty^{2}(d-2)$-cycles differing very little from $\Gamma_{d-2}$, and the value of (8) extended over $\Gamma_{d}^{\prime}$ is equal to the constant period of (9) relatively to $\Gamma_{d-2}$ integrated over a finite two-dimensional continuum of the complex $\left(x_{1}, x_{2}\right)$ space, and therefore again zero.

We have thus obtained $R_{d-2}^{\prime} d$-cycles with respect to which (8) has no periods. The same reasoning may be pursued by replacing (8), $V_{d}$ and $\Gamma_{d}$ by (9), $H^{2}$ and $\Gamma_{d-2}$, and so on. We shall have finally

$$
R_{d-2}^{\prime}+R_{d-6}^{\prime}+\cdots=R_{d-2}-R_{d-4}+R_{d-6} \cdots
$$

non-effective d-cycles relatively to which integrals of the first kind have no periods. Similarly there are $R_{s-2}-R_{s-4}+\cdots$ non-effective $s$-cycles with respect to which $s$-uple integrals of the first kind have no periods. In these expressions the last term is either $\pm R_{1}$ or $\pm R_{0}= \pm 1$.

16. It is easy to show that $s$-tuple integrals of the first kind have no periods relatively to the $\rho_{s}$ cycles of No. 8. To show this, transform $V_{d}$ birationally into a variety $V_{d}^{\prime}$ with ordinary singularities in such a way that the transformed $A_{k}^{\prime}$ of $A_{k}$ be part of the hyperplane section $x_{1}=0$. The finite cycles contained in the ( $A_{k}^{\prime}$ )'s will now yield zero for period since at every point of them $d x_{1}=0$. The same is true for finite cycles of $V_{d}$ that have become infinitesimal on $V_{d}^{\prime},-$ a circumstance that may well happen-, for these infinitesimal cycles may be assumed at finite distance also. Thus there will be in all $\rho_{s}+R_{s-2}-R_{s-4}+R_{s-6} \cdots$ distinct s-cycles relatively to which integrals of the first kind have no periods.

Are there any others with the same property? Probably not, though we have succeeded in proving it only for algebraic surfaces and for abelian varieties with a period matrix as general as possible. 


\section{Chapter 2. Invariants $\rho, \sigma$ of algebraic varieties}

\section{§1. Poincaré's normal functions for algebraic surfaces}

17. Let $F(x, y, z)=0$ represent an algebraic surface $V_{2}$, of order $m$, irregularity $\frac{1}{2} R_{1}=q$, and denote by $|H|$ and $p$ the system of plane sections and the genus of a generic $H$. As is well known, a set of $p$ independent integrals of the first kind of $H_{y}$, arbitrary curve of the pencil $\left\{H_{y}\right\}$, can be obtained thus: For the first $p-q$ integrals we take

$$
u_{h}=\int \frac{P_{h}(x, y, z) d x}{F_{2}^{\prime}} \quad(h=1,2, \cdots, p-q),
$$

where $P_{h}$ is an adjoint polynomial of order $m-3$, and for the remaining we take the $q$ integrals of the first kind $u_{p-q+1}, u_{p-q+2}, \cdots, u_{p}$ of $V_{2}$. Let $A_{1}, A_{2}, \cdots$, $A_{m}$ be the base points of $\left\{H_{y}\right\}, C$ any algebraic curve of $V_{2}, M_{1}, M_{2}, \cdots, M_{n}$ the variable points of the group $\mathrm{CH}_{y}$ or these points associated with some of the $(A)$ 's. Consider the abelian sums

$$
v_{h}(y)=\sum_{j=1}^{n} \int_{A_{1}}^{\boldsymbol{N}_{j}} d u_{h} \quad(h=1,2, \cdots, p),
$$

first introduced by Poincare,, who gave for them expressions which the present choice of integrals of the first kind reduces to

$$
\begin{array}{lrl}
v_{h}(y)=\sum_{k=1}^{N} \frac{\lambda_{k}}{2 \pi i} \int_{b}^{b_{k}} \frac{\Omega_{h k}(Y)}{Y-y} d Y & (h=1,2, \cdots, p-q), \\
v_{p-q+\varepsilon}=\beta_{s} & (s=1,2, \cdots, q) .
\end{array}
$$

In these expressions the $(\lambda)$ 's are integers, called characteristic integers, $b$ arbitrary, $b_{1}, b_{2}, \cdots, b_{N}$ the critical values of $y$ for $\left\{H_{y}\right\}$, the $(\beta)$ 's constants, and finally $\Omega_{h k}$ the period of $u_{h}$ relatively to the cycle of $H_{\nu}$ that vanishes for $y=b_{k}$. The corresponding periods of $u_{p-q+\varepsilon}$ are obviously zero. The paths of integration form a system of non-intersecting cuts in the $y$ plane.

In the expressions as given by Poincare there appear certain rational functions at the right. These expressions can only be polynomials, for their infinities are the same as those of the $(\dot{u})$ 's. Moreover, by means of the birational space transformation $x=x^{\prime} / y^{\prime}, y=1 / y^{\prime}, z=z^{\prime} / y^{\prime}$, we may easily verify that $v_{h}(y)^{\prime}, h \leqq p-q$, vanishes at infinity, while the other $(v)$ 's are finite there, hence the form of the expressions (1) follows. Besides it can be shown directly that $v_{p-q+\varepsilon}$ is constant for it is uniform in $y$ and finite everywhere (Severi).

* Annales de l'Ecole Normale supérieure, ser. 2, vol. 27 (1910), pp. 55-108. Sitzungsberichte der Berliner mathematischen Gesell s ch a f , vol. 10 (1911), pp. 28-55. 
18. We obtain a first set of relations to be satisfied by the quantities entering in the $(v)$ 's when we express the fact that the $(v)$ 's are independent of $b$ or that $\partial v_{k} / \partial b=0$.

If in the relation obtained we replace $b$, which is arbitrary, by $y$, it becomes

$$
\sum_{k=1}^{N} \lambda_{k} \Omega_{h k}(y)=0 \quad(h=1,2, \cdots, p-q) .
$$

This condition once satisfied, the ( $v$ )'s as given by (1) are regular everywhere, infinity included, except at the critical points $b_{i}$, and this is as it should be.

Another condition is obtained thus: Let there be a value $y=\alpha$ for which

$$
\sum_{\lambda=1}^{p-q} \frac{\gamma_{h} P_{h}(x, y, z)}{(y-\alpha)^{s}}
$$

remains finite, the $(\gamma)$ 's being constants. Then $\sum \gamma_{h} v_{h}(y) \cdot(y-\alpha)^{-s}$ must also remain finite. We can always select our integrals so that $s=1$ for all the $(\alpha)$ 's (Poincaré), but this is immaterial.

A set of functions satisfying these two conditions and behaving like the $(v)$ 's at the critical points forms what Poincaré has called a set of normal functions. He has proved the following fundamental theorem: To a set of normal functions always corresponds an algebraic curve for which they are Abelian sums. Exceptionally, the curve is reduced to one or more of the points $A_{i}$.

Given two algebraic curves $B, C$, of $V_{2}$, we will write with Severi $B=C$, if there exists an algebraic curve $E$ such that $B+E, C+E$ are both total curves of the same continuous system.* When $B$ varies in its continuous system the characteristic integers corresponding to the total group $B H_{y}$ are fixed. They are only determined however up to a system of integers corresponding to a period, as the periods obviously form sets of normal functions. The set of characteristic integers of $B+E$ is obtained by adding the corresponding integers of $B$ and $E$. Hence if $B=C$, the differences between their characteristic integers form a set corresponding to a period, it being understood that characteristic integers are taken for the total groups $B H_{y}$, $\mathrm{CH}_{y}$.

\section{§ 2. Algebraic cycles of a surface. Fundamental theorem}

19. We propose first to interpret the conditions that the $(v)$ 's must satisfy from a somewhat different viewpoint.

We begin with the second condition. When $\sum \gamma_{h} P_{h}(x, y, z)=(y-\alpha)$. $Q(x, y, z), Q$ is an adjoint polynomial of order $m-4$ and the double integral,

$$
\iint \frac{Q(x, y, z)}{F_{z}^{\prime}} d x d y
$$

* These systems may be reducible but must be connected. A thorough treatment of this point has been given by Albanese, A n a li d i m a te m a t i a , ser. 3, vol. 24 (1915), pp. 159-233. Our "equivalence" is the same as his "virtual equivalence". 
is of the first kind. Let $\omega_{k}(y)$ be the period of

$$
\int \frac{Q(x, y, z)}{F_{2}^{\prime}} d x
$$

corresponding to $\Omega_{h k}$ for $u_{h}$. We must have

$$
\sum_{h, k} \gamma_{h} \lambda_{k} \int_{b}^{b_{k}} \frac{\Omega_{h k}(Y) d Y}{Y-\alpha}=\sum_{k} \lambda_{k} \int_{b}^{b_{k}} \omega_{k}(y) d y=0 .
$$

Conversely if (3) is of the first kind,

$$
\int \frac{(y-\alpha) Q(x, y, z)}{F_{2}^{\prime}} d x
$$

is a linear combination of $u_{1}, u_{2}, \cdots, u_{p-q}$, identically zero for $y=\alpha$, and the relation (5) corresponding to it must be satisfied. Hence (5) is true whatever the integral of the first kind (3) considered.

20. Let $\Gamma$ be any two-cycle of $V_{2}$. We may assume without loss of generality that any $H_{y}$ meets it in a finite number of points since this can always be obtained by slightly deforming the cycle if necessary. Since $\Gamma$ is two sided we may sense it by assigning a positive direction of rotation for its small circuits. Let now $y$ describe a small positive circuit in its plane. Of the points of intersection of $\Gamma$ and $H_{y}$, a certain number, say $n^{\prime}$, will describe positive circuits on $\Gamma$, while the $n^{\prime \prime}$ remaining points will do just the opposite. The number $n^{\prime}-n^{\prime \prime}$ does not depend upon the position of the circuit in the $y$ plane, for when a point of one type disappears one of the other disappears at the same time. The number $n^{\prime}-n^{\prime \prime}$ is therefore a definite simultaneous character of the two manifolds $\Gamma, H_{y}$. Poincaré introduced it for manifolds $M_{s}, M_{k-s}$ in an $M_{k}$ (the indices indicate the dimensionality), and he denoted it by $N\left(M_{s} M_{k-s}\right)$. The $N$ really is superfluous, and we shall simply write $\left(M_{s} M_{k-s}\right)$, or $\left(\Gamma H_{y}\right)=n^{\prime}-n^{\prime \prime}$. All we need to know concerning it here is that if $\Gamma \sim 0$, also $\left(\Gamma H_{y}\right)=0$.

We observe at once that if $\Gamma$ is an algebraic cycle $(C)$, of the two numbers $n^{\prime}, n^{\prime \prime}$, one is necessarily zero, the other being equal to $\pm\left[\mathrm{CH}_{\nu}\right]$, or in absolute value equal to the order of $C$. This follows from the well-known property of a multiply sheeted Riemann surface, that when the independent variable describes a small circuit in its plane, the corresponding points in the various sheets describe circuits sensed alike relatively to the surface. We shall make once for all the convention that $(C)$ is to be so sensed that $\left(\mathrm{CH}_{y}\right)=\left[\mathrm{CH}_{y}\right]$.

Returning to the cycle $\Gamma$, let us join $A_{1}$ to all the points of intersection of $\Gamma$ and $H_{y}$ by lines in $H_{y}$. Their locus as $y$ describes its plane without crossing the cuts $b b_{k}$ is a manifold $M_{3}$ whose boundary is composed of $\Gamma$, of part or all of $H_{y}$, and of a manifold $M_{2}$ that may be described thus: When $y$ goes from 
$y_{0}$ on one side of $b b_{k}$ to the point $y_{0}^{\prime}$ right opposite on the other, by turning around $b_{k}$ without crossing the cut, the aggregate of lines whose locus is $M_{3}$ returns to a new position in $H_{y_{0}}=H_{y_{0}^{\prime}}$, which differs from the old position by a multiple of the linear cycle $\delta_{k}$ of $H_{y}$ that reduces to a point when $y=b_{k}$. The locus of $\delta_{k}$ when $y$ describes $b b_{k}$ is a two-dimensional manifold $\Delta_{k}$, somewhat similar to a cone with its vertex at the point of contact of the plane $y=b_{k}$, and its base in the position of $\delta_{k}$ in $H_{b}$, and we have

$$
\begin{gathered}
M_{2}=-\sum \lambda_{k} \Delta_{k} . \\
\Gamma \sim \sum \lambda_{k} \Delta_{k}+\text { part of }\left(H_{b}\right) .
\end{gathered}
$$

A question may be raised as to this discussion: The difference $n^{\prime}-n^{\prime \prime}$ is constant, but $n^{\prime}, n^{\prime \prime}$, may vary. Let $\zeta$ be a line separating regions in the $y$ plane with different values of these integers, so that along $\zeta$ some of the points $B_{i}^{\prime}$ of one type, coincide with as many, $B_{i}^{\prime \prime}$, of the other. Is the $M_{2}$ locus of the lines $A_{1} B_{i}^{\prime}, A_{1} B_{i}^{\prime \prime}$, when $y$ describes $\zeta$, part of the boundary of $M_{3}$ ? We may deform $\zeta$, without making it cross the critical points, so as to reduce it to a sum of the loops $b b_{k}$. This amounts to deforming the given cycle into one for which the $M_{2}$ here considered is reduced to a sum of the manifolds $\Delta_{k}$, which still leads to a relation such as (6).

To return to our problem, in the particular case where $\Gamma=(C)$, the mode of derivation of the $(v)$ 's shows at once that the coefficients $\lambda_{k}$ in (6) are the characteristic integers of the curve $C$. Now from the theorem of PoincaréCauchy for functions of several variables it follows at once that the periods of an integral of the first kind relatively to homologous cycles are equal. But (3) extended over part of $\left(H_{b}\right)$ gives zero, hence

$\iint_{(C)} \frac{Q(x, y, z)}{F_{z}^{\prime}} d x d y=\sum \lambda_{k} \iint_{\Delta_{k}} \frac{Q(x, y, z)}{F_{z}^{\prime}} d x d y=\sum \lambda_{k} \int_{b}^{b_{k}} \omega_{k}(y) d y$.

The relation (5) means then that the period of (3) relatively to the algebraic cycle $(C)$ is zero, in accordance with $\mathrm{Ch}$. I. Thus the conditions satisfied by the $(\lambda)$ 's express merely that integrals of the first kind have no periods relatively to the algebraic cycles.

21. The converse of the property just obtained constitutes the

Fundamental Theorem: $A$ two-cycle $\Gamma$ without periods of integrals of the first kind is algebraic.

For if $\Gamma$ satisfies (6), the corresponding period of (3) is

$$
\sum \lambda_{k} \int_{b}^{b_{k}} \omega_{k}(y) d y
$$

and since it vanishes there is an algebraic curve $C$ such that to the variable part of $\mathrm{CH}_{\nu}$ or to this part associated with some of the points $A_{i}$ correspond 
the characteristic integers $\lambda_{k}$. Let $\mu_{k}^{i}$ be the $k$ th characteristic integer of $A_{i}$. There is a certain two-cycle $\Gamma^{i}=\sum \mu_{k}^{i} \Delta_{k}+$ part of $\left(H_{b}\right) \sim 0$ forming the boundary of the locus of $A_{1} A_{i}$ as $y$ describes its plane under the same conditions as above, and the $\left(\mu^{i}\right)$ 's are the characteristic integers of the normal functions corresponding to $A_{i}$. We may find integers $t_{i}$ such that $\Gamma+\sum t_{i} \Gamma^{i}$ $\sim \Gamma \sim \sum_{k}\left(\lambda_{k}+\sum_{i} t_{i} \mu_{k}^{i}\right) \Delta_{k}+$ part of $\left(H_{b}\right)$, the coefficients of the $(\Delta)$ 's at the right being now the characteristic integers of the total group $\mathrm{CH}_{y}$.

We may assume $C$ to be variable in a continuous system with no fixed base points at the ( $A$ )'s for, if this is not so, we may replace $\Gamma$ and $C$ by $\Gamma+t(H)$ and $C+t H$, and with $t$ sufficiently great the assumption will certainly be satisfied, especially since the $(A)$ 's are after all arbitrary points of the surface. Let $C^{\prime}$ be a curve of the same system as $C$ and not going through the $(A)$ 's. $C$ and $C^{\prime}$ have the same characteristic integers, hence

$$
\left(C^{\prime}\right) \sim \sum_{k}\left(\lambda_{k}+\sum_{i} t_{i} \mu_{k}^{i}\right) \Delta_{k}+\text { part of }\left(H_{b}\right) .
$$

$\left(C^{\prime}\right)-\Gamma$ is then $\sim$ to a part of $\left(H_{b}\right)$, and if, as it is proper to assume, $H_{b}$ is irreducible, $\left(C^{\prime}\right)-\Gamma \sim(t H)$, which proves our theorem.

22. Given two algebraic curves $C, D$ of $V_{2}$, if $C=D$, then also $(C) \sim(D)$. For if $E$ is such that $C+E$ and $D+E$ are total curves of the same continuous system the cycles $(C+E)$ and $(D+E)$, reducible to each other by continuous deformation, are homologous, whence at once $(C) \sim(D)$.

I say that conversely if $(C) \sim(D)$, then also $C=D$. For we have then

$$
\left((C-D) H_{y}\right)=\left(C H_{y}\right)-\left(D H_{y}\right)=\left[C H_{y}\right]-\left[D H_{y}\right]=0,
$$

and therefore $C, D$ have the same order.

The axes being arbitrary, we may assume that une curves do not go through the $(A)$ 's. Then since $(C) \sim(D)$ their characteristic integers are equal, and so are their normal functions $v_{h}, h \leqq p-q$. We can add to $C$ any curve of the same continuous system as $t H$, and take $t$ so great that the complete continuous systems determined by $C+t H$ and $D+t H$ contain $\infty$ linear systems. It may as well then be assumed that $C, D$ already satisfy this condition. We may therefore choose a curve $C^{\prime}=C$ not passing through the $(A)$ 's and for which the $\left(v_{p-q+k}\right)$ 's are the same as for $D$. As $C^{\prime}$ and $D$ have already the same normal functions of index $\leqq p-q$, these functions will all be the same for both. Since the orders are equal, $C^{\prime}$ and $D$ are total curves of the same linear system (Poincaré), and therefore $C^{\prime}=D=C$, as was to be proved.

More generally the two relations $\sum t_{i}\left(C_{i}\right) \sim 0, \sum t_{i} C_{i}=0$ are equivalent. For by writing $t_{i}=t_{i}^{\prime}-t_{i}^{\prime \prime}$, where $t_{i}^{\prime}$ and $t_{i}^{\prime \prime}$ are non-negative integers, they become $\sum t_{i}^{\prime}\left(C_{i}\right) \sim \sum t_{i}^{\prime \prime}\left(C_{i}\right), \sum t_{i}^{\prime} C_{i}=\sum t_{i}^{\prime \prime} C_{i}$, and these relations owing to the positive sign of all coefficients are obviously equivalent.

Trans. Am. Math. Soc. 23. 
Remark. Since $(C H) \neq 0,(C)$ is non-effective relatively to $H$ if $C$ is not a virtual curve. More generally, $(C)$ is non-effective relatively to a curve belonging to a sufficiently general linear system.

23. A zero divisor $\Gamma$ for two cycles is algebraic (No. 21). Since it can be considered as the difference between two algebraic cycles $(C)+\Gamma$ and $(C)$, the corresponding algebraic curve can be considered as the difference between two curves $D$ and $C$. If $\lambda \Gamma \sim 0$ for some integer $\lambda>1$, but not for $\lambda=1$, then also $\lambda(D-C)=0$, this being untrue for $\lambda=1$, so that $D-C$ is a zero divisor for the curves. Conversely if $D-C$ is a zero divisor for the curves, $(D)-(C)$ is a zero divisor for the two-cycles. If then we introduce with Severi virtual curves, there exists a holoedric isomorphism between the operations $+C$ applied to the curves and $+(C)$ applied to the cycles. We recall that, as shown by Picard and Severi, there is a maximum $\rho$ of the number of algebraically independent curves of $V_{2}$, and that Severi has denoted by $\sigma$ the order of the Abelian group formed by the zero divisors for algebraic curves, so that there are exactly $\sigma-1$ such divisors. According to what precedes, the number $\rho$ is therefore equal to the number of distinct two-cycles without periods of integrals of the first kind, and $\sigma$ is equal to the torsion indices $\sigma_{1}, \sigma_{2}$ (equal according to Poincaré).

24. The case when the geometrical genus $p_{0}=U$ leads at once to an interesting result. For then any cycle is algebraic, hence $R_{2}=\rho$,

$$
\rho_{0}=\rho_{0}^{2}=R_{2}-\rho=0 \text {. }
$$

Thus if $p_{0}=0, \rho_{0}=0$, that is, if an algebraic surface is without double integrals of the first kind it is also without double integrals of the second kind. This has already been proved by Bagnera and de Franchis for irregular surfaces and for some regular surfaces, ${ }^{*}$ and there is a remark by Poincare at the end of his mémoire in the Annales de l'Ecole Normale which easily leads to the same result.

25. Assume $p_{o}>0$ and consider $p_{o}$ independent integrals of the first kind

$$
\iint \frac{Q_{h}(x, y, z)}{F_{z}^{\prime}} d x d y \quad\left(h=1,2, \cdots, p_{0}\right) .
$$

We must have

$$
\sum_{k=1}^{N} \lambda_{k} \int_{b}^{b_{k}} \omega_{h k}(y) d y=0 \quad\left(h=1,2, \cdots, p_{0}\right),
$$

where $\omega_{h k}$ corresponds to $\omega_{k}$ for (3). Are these relations distinct? If they were not there would have to exist constants $c_{h}$ not all zero such that

$$
\sum_{h=1}^{p_{k}} c_{h} \int_{b}^{b_{k}} \omega_{h k}(y) d y=0 \quad(k=1,2, \cdots, N),
$$

*Rendicontidel Circolo Matematicodi Palermo, vol. 30 (1910), pp. 1S5-238. 
and therefore an integral of the first kind

$$
\sum c_{h} \iint \frac{Q_{h}(x, y, z)}{F_{z}^{\prime}} d x d y
$$

without periods. If we assume that this is impossible the $p_{o}$ relations obtained are all distinct.

26. We are thus led to inquire: Can a double integral of the first kind be without periods? It is very likely that this question must be answered in the negative though we have succeeded in proving it only in some very special cases. For the present we merely wish to establish two relations which must exist in case the answer were affirmative.

If (3) is without periods we must have (Picard),

$$
\frac{Q(x, y, z)}{F_{z}^{\prime}}=\frac{\partial}{\partial x}\left(\frac{A(x, y, z)}{\phi(u) F_{z}^{\prime}}\right)+\frac{\partial}{\partial y}\left(\frac{B(x, y, z)}{\phi(u) F_{z}^{\prime}}\right),
$$

where $A, B$ are polynomials adjoint to $V_{2}$ and $\phi$ is a polynomial in

$$
u=a x+b y+c z,
$$

an arbitrary linear form in $x, y, z$. In fact this result has been established by Picard solely for $u=y$, but the extension is at once obtained by a transformation of coördinates.

Let $U, V$ denote the functions in parenthesis at the right and let us introduce with Picard the integral of total differentials

$$
\int(-U+\epsilon) d y+V d x ; \quad \epsilon=\int^{x, 2} \frac{Q(x, y, z)}{F_{z}^{\prime}} d x
$$

It has most of the properties of those with algebraic integrands since $\epsilon$ is holomorphic everywhere except in the vicinity of the planes $y=b_{k}$, and there only fails to be so for certain determinations. We may reproduce almost verbatim a discussion of Severi's relating to integrals of total differentials of the second kind.* In particular we may subtract an integral $\int-R d y+S d x$ of total differentials with rational integrands $R, S$, so as to suppress the curves of infinity other than $y=b_{i}$ and also the periods with respect to the linear cycles of $V_{2}$. We will then have

$$
\frac{Q}{F_{z}^{\prime}}=\frac{\partial}{\partial x}(U-R)+\frac{\partial}{\partial y}(V-S)=\frac{\partial}{\partial x} \frac{A(x, y, z)}{F_{z}^{\prime}}+\frac{\partial}{\partial y} \frac{B(x, y, z)}{F_{z}^{\prime}},
$$

where $A, B$ are adjoint polynomials, and now the Abelian integral

$$
\int\left(\frac{1}{F_{z}^{\prime}}\right)(A d y-B d x)
$$

* Mathematische Annalen, vol. 61 (1905), pp. 20-49. 
when attached to any algebraic curve $C$ of $V_{2}$, becomes one of the first kind for that curve. Its periods with respect to an invariant cycle in a plane section are zero. It follows that it possesses all the properties of an integral of total differentials which do not require the use of the conditions of integrability in their proof. In particular we have $A=x E+A_{1}, B=y E+B_{1}$, where $A_{1}, B_{1}, E$ are polynomials of order $m-3$. However, since the invariant periods are now zero we may take $E$, and therefore $A_{1}$ and $B_{1}$, to be adjoint polynomials, which is certainly not the case for an integral of total differentials. Finally it may be shown that there exists an adjoint polynomial $C(x, y, z)$ such that

$$
A F_{x}^{\prime}+B F_{y}^{\prime}+C F_{z}^{\prime} \equiv D F, \quad A_{x}^{\prime}+B_{v}^{\prime}+C_{z}^{\prime}+D \equiv Q Q .
$$

These are the relations which we wished to derive. We observe that

$$
\iint \frac{Q(x, y, z)}{F_{z}^{\prime}} d x d y=\iint \frac{Q(x, y, z)}{F^{\prime}{ }_{y}} d z d x=\iint\left[\frac{\partial}{\partial x} \frac{A}{F_{y}^{\prime}}+\frac{\partial}{\partial y} \frac{B}{F^{\prime}{ }_{y}}\right] d z d x \text {, }
$$

and hence $C=z E+C_{1}$ where $C_{1}$ is adjoint of order $m-3$.

By introducing homogeneous variables we may replace the necessary conditions for the existence of a double integral of the first kind without periods (7) by

$$
A F_{x}^{\prime}+B F_{y}^{\prime}+C F_{z}^{\prime}+D F_{t}^{\prime} \equiv 0, \quad A_{x}^{\prime}+B_{y}^{\prime}+C_{z}^{\prime}+D_{t}^{\prime} \equiv Q,
$$

where $A, B, C, D$ are polynomials of order $m-3$. We verify at once by means of a projective transformation that $\alpha A+\beta B+\gamma C+\epsilon D=0$ cuts out on the plane $\alpha x+\beta y+\gamma z+\delta t=0$ an adjoint of order $m-3$ to the section of $V_{2}$ by the plane. Let us assume then that $(0,0,0,1)$ is a point on the double curve. The surfaces $A=B=C=0$ go through this point, hence it is a double point for $A F_{x}^{\prime}+B F_{y}^{\prime}+C F_{z}^{\prime}=0$, and therefore for $D F_{t}^{\prime}=0$, which shows that $D=0$ also goes through the point. Hence $A, B, C, D$ are adjoint polynomials of order $m-3$.

27. In the whole discussion of this chapter it is possible to replace $|H|$ by any simple linear system $|E|, \infty^{2}$ at least, irreducible and such that when an $E$ acquires a new singularity this consists in general in an ordinary double point. We simply take a generic pencil $\left\{E_{u}\right\}$ and replace everywhere $y$ by $u$, the modifications being insignificant.

By considering an adequate $|E|$ on a $V_{2}$ with arbitrary singularities our results can be extended to it. Since $\rho_{0}=R_{2}-\rho$ is an absolute invariant the cycles which are gained or lost by a birational transformation are all algebraic as we already had occasion to state.

If $|E|$ is not general enough much, if not all, of the discussion is still valid. However, one may be led to neglect certain infinitesimal cycles and thus be led to the numbers $[\rho],[\sigma]$ instead of $\rho, \sigma$. 
In fact all this holds whenever we deal with a rational or irrational pencil such that a certain point $A_{1}$ may be uniquely determined on the generic curve of it. We can thus derive Severi's results as to the base for a surface which represents the pairs of points of two algebraic curves. The Abelian sums such as in $\S 1$ lead at once to Hurwitz's equations for correspondences between the two curves.

28. We have studied more especially the relations between the numbers $\rho, \sigma$ and certain cycles. The one to one correspondence between algebraic cycles and curves shows that to a minimum base for the cycles corresponds a minimum base for the curves and conversely. But the curves of a minimum base may well be virtual. It may be shown that from a base containing such curves we may always derive one composed exclusively of effective curves. There will be an effective minimum base composed of $\rho+\sigma-1$ curves if $\rho>1$, and of $\sigma$ curves for $\rho=1$, and in both cases an ordinary effective base composed of $\rho$ curves (Severi).

\section{$\S 3$. Extension to any $V_{d}$}

29. For the sake of simplicity let us take $d=3$. Let then $F(x, y, z, t)=0$ be the equation of an irreducible $V_{3}$, with ordinary singularities, of order $m$, irregularity $\frac{1}{2} R_{1}=q$ and genus of the plane sections equal to $p$. We designate again by $|H|$ the system of hyperplane sections, and will call $A_{1}, A_{2}, \cdots, A_{m}$ the fixed points of the curves $H_{y} H_{z}$. As before we shall have $p$ integrals of the first kind attached to these curves of which $p-q$ are

$$
u_{h}=\int \frac{P_{h}(x, y, z, t)}{F_{t}^{\prime}} d x \quad(h=1,2, \cdots, p-q),
$$

where $P_{h}$ is an adjoint polynomial of order $m-3$ in $x, y, t$, and the $q$ remaining integrals $u_{p-q+1}, u_{p-q+2}, \cdots, u_{p}$ are those of total differentials of the first kind of $V_{3}$ (Castelnuovo-Enriques).

Let $C$ be an algebraic surface of $V_{3}, M_{1}, M_{2}, \cdots, M_{n}$ the variable points of the group $\mathrm{CH}_{y} \mathrm{H}_{z}$ or these points associated with some of the $(A)$ 's. We have

$$
\begin{aligned}
v_{h}(y, z) & =\sum_{j=1}^{n} \int_{A}^{M s} d u_{h}=\sum_{k=1}^{N} \frac{\lambda_{k}}{2 \pi i} \int_{b}^{b_{k}} \frac{\Omega_{h k}(Y, z) d Y}{Y-y}, \\
v_{p-q+j}=\beta_{j} & (h=1,2, \cdots, p-q ; j=1,2 ; \cdots, q) .
\end{aligned}
$$

In these expressions the ( $\left.b_{k}\right)$ 's are the critical values of $y$ for the pencil $\left\{H_{y} H_{z}\right\}$ of $H_{2}$ and the $(\beta)$ 's are constants. Let us consider an arbitrary double integral of the first kind of $H_{z}$ of the type $\int \mathcal{S}\left(1 / F_{t}^{\prime}\right) Q(x, y, z, t) d x d y$, where $Q$ is an adjoint polynomial of order $m-4$ in $x, y, t$. It may be easily shown that any integral of the first kind of $H_{z}$ is linearly dependent upon 
those of this type. We must then have

$$
\sum \lambda_{k} \int_{b}^{b_{k}} \omega_{k}(y, z) d y=0, \quad \sum \lambda_{k} \omega_{k}(y, z) \equiv 0,
$$

the $(\omega)$ 's having always the same meaning. But besides these conditions the following must also be fulfilled: When $z$ describes a closed path the set of $(\lambda)$ 's is in general returned to a different set composed of $\left(\lambda^{\prime}\right)$ 's, the differences $\lambda_{k}-\lambda_{k}^{\prime}$ forming the set of characteristic integers corresponding to the variations of the cycle $\left(\mathrm{CH}_{z}\right)$ in the manner of No. 20. As this variation is $\sim 0$, or, if we please, as $\mathrm{CH}_{z}$ is a uniquely determined curve on each $H_{z}$, the differences $\lambda_{k}-\lambda_{k}^{\prime}$ must form a set corresponding to a system of periods, and this must be true whatever the closed path described by $z$.

Conversely, if all these conditions are fulfilled there exists an algebraic surface $C$ corresponding to the functions $v_{h}(y, z)$. Indeed, for every $z$ these functions determine in a unique way, that is, rationally in terms of $z$, a certain linear system of curves on $H_{z}$. By imposing an adequate behavior at the $(A)$ 's we may always determine in a unique manner in this linear system, a curve the locus of which will be a surface $C$. More explicitly, the curve in $H_{y}$ will be the intersection of the surface $F(x, y, z, t)=0$ of the space $(x, y, t)$ with other surfaces $\Phi(x, y, t)=0$, where these various surfaces have no other curves in common. The $(\Phi)$ 's however may be taken to be polynomials with coefficients rational in $z$, whence the existence of the surface $C$ follows immediately.

30. Let $C_{1}, C_{2}$, be two algebraic surfaces of $V_{3}$. If $\left(C_{1}\right) \sim\left(C_{2}\right), \bmod . V_{3}$, $\left(C_{1} H_{2}\right) \sim\left(C_{2} H_{2}\right)$ mod. $H_{2}$, and therefore also mod. $V_{3}$. It follows that

$$
C_{1} H_{z}=C_{2} H_{z}
$$

in $H_{2}$ and consequently $C_{1}$ and $C_{2}$ are of the same order and have the same set of characteristic integers.

We may find two surfaces $D^{\prime}, D^{\prime \prime}$, belonging to a continuous system with $\infty^{q}$ linear systems and such that $C_{1}+D^{\prime}$ and $C_{2}+D^{\prime \prime}$ belong totally to the same linear system, whence at once $C_{1}=C_{2}$, this relation having a sense similar to that given to it for a $V_{2}$. Thus if $\left(C_{1}\right) \sim\left(C_{2}\right)$, then also $C_{1}=C_{2}$. The converse is obviously true. We can see then that the relations

$\sum t_{i}\left(C_{i}\right) \sim 0 \bmod . V_{3}, \sum t_{i}\left(C_{i} H_{z}\right) \sim 0 \bmod . H_{z}, \sum t_{i} C_{i} H_{z}=0, \sum t_{i} C_{i}=0$, are all equivalent. ${ }^{*}$ From the existence of minimum and ordinary bases

* See a different proof of the equivalence of the relations $C_{1} H_{3}=C_{2} H_{3}$ and $C_{1}=C_{2}$ given by Severi in the At ti del Rea le Inst it u to Veneto, vol. 75 (1916), p. 1138. He had already stated this proposition without proof at the end of his memoir of the A n n a les de l'Ecole Normale superieure, loc. cit.-The question may be raised as to the sense to be assigned to the algebraic four-cycle $(C)$. We know (No. 20) the sense to be assigned to the cycle $\left(\mathrm{CH}_{3}\right)$. The four-cycle in question is then to be considered as the locus of $\left(\mathrm{CH}_{\mathrm{s}}\right)$ properly sensed associated to the $z$-plane sensed in a definite manner. 
for the cycles follows the existence of similar bases for the curves. The yariety will have numbers $\rho, \sigma$ and if $\rho^{\prime}, \sigma^{\prime}$ are those of $H$, then $\rho \leqq \rho^{\prime}, \sigma \leqq \sigma^{\prime}$, whatever $H$ may be.

31. The periods of the double integral attached to $H_{3}$

$$
\iint \frac{P(x, y, z, t)}{F_{t}^{\prime}} d x d y,
$$

where $P$ is the adjoint polynomial, are of the form

$$
\sum_{k=1}^{N} \lambda_{k} \int_{b}^{b_{k}(z)} \Omega_{k}(y, z) d y .
$$

Let $z=c$ be a value $b$ for which two of the upper limits, say $b_{1}$ and $b_{2}$, coincide. The author has shown (Paris $\mathrm{Comptes} \mathrm{R} \mathrm{e} \mathrm{m} \mathrm{u}$ s, loc. cit.) that when $z$ turns around $c,(10)$ is increased by a multiple of the period

$$
\int_{b}^{b_{1}} \Omega_{1}(y, z) d y-\int_{b}^{b_{3}} \Omega_{2}(y, z) d y ; \quad \Omega_{1}=\Omega_{2} .
$$

This will be true whether (10) is a period or not. If we develop in series of powers of $Y^{-1}$ the differential coefficient of

$$
\iint \frac{P(x, y, z, t)}{(y-Y) F_{t}^{\prime}} d x d y
$$

and apply this to each term of the series we find that the period

$$
\sum \lambda_{k} \int_{b}^{b_{k}} \frac{\Omega_{k}(y, z)}{y-Y} d y
$$

increases by a multiple of

$$
\int_{b}^{b_{1}} \frac{\Omega_{1}(y, z)}{y-Y} d y-\int_{b}^{b_{2}} \frac{\Omega_{2}(y, z)}{y-Y} d y .
$$

In particular, for $|y|$ great enough, $v_{h}(y)(h \leqq p-q)$ is increased by a multiple of

$$
\frac{1}{2 \pi i} \int_{b}^{b_{1}} \frac{\Omega_{h 1}(Y, z)}{Y-y} d Y-\frac{1}{2 \pi i} \int_{b}^{b_{2}} \frac{\Omega_{h 2}(Y, z)}{Y-y} d Y .
$$

I say that this and the similar expressions are not submultiples of the periods of $u_{h}$. For it vanishes for $z=c$ and if it were a submultiple of the periods the $(u)$ 's would lose a period and $H_{y} H_{z}$ would lose a linear cycle for $z=c$, $y$ arbitrary, which is certainly not the case if $\left\{H_{z}\right\}$ is generic in $|H|$.

It follows at once that the zero divisors for the curves of $H_{z}$ are invariant. For otherwise a multiple of the increments of some set of $(v)$ 's when $z$ turns around $c$ would form a period, hence this would be true for all sets of $(v)$ 's in direct contradiction to what has just been found. 
Thus the zero divisors for the curves of $H_{z}$ are also zero divisors of $V_{3}$, whence $\sigma \equiv \sigma^{\prime}$, and therefore finally $\sigma=\sigma^{\prime}$, that is, $V_{3}$ and its hyperplane sections have equal invariants $\sigma$. As they have equal invariants $\sigma_{1}$, it follows that $\sigma=\sigma_{1}$. Here we cannot affirm any more that $\sigma=\sigma_{2}$. This is due to the possible existence in $H_{z}$ of two-cycles not zero divisors for it but zero divisors for $V_{3}$.

32 . We pass to the proof of a theorem specially important in the determination of $\rho, \sigma$, for the surfaces in $V_{3}$.

Let $c_{1}, c_{2}, \cdots, c_{\nu}$ be the critical values of $z$ for $\left\{H_{z}\right\}$. For each of these two of the $(b)$ 's and no more coincide. Let us make the pencil vary continuously in a net $\Sigma$ containing it. The $(C)$ 's will be displaced on an irreducible curve $D$ if as we shall assume $\Sigma$ is arbitrary in $|H|$, for when $V_{3}$ assumed in an $S_{4}$ is transformed by reciprocal polars, $D$ is changed into a plane section of the transformed variety.

To every point $c_{j}$ corresponds a well-defined two-cycle $\Gamma_{j}$ of $H_{z}$ which becomes zero for $z=c_{j}$ and such that when $z$ turns around $c_{j}$ any other cycle is increased by a multiple of it. I say that if one of these cycles is algebraic so ore the others. For $\left\{H_{z}\right\}$ may be made to vary within $\Sigma$ so as to permute $c_{j}$ with $c_{k}$, thus bringing $\Gamma_{j}$ assumed within an $H_{z}$ very near $H_{c_{j}}$ into $\Gamma_{k}$ within an $H_{z}$ very near $H_{c_{k}}$. Noticing that the two extreme surfaces have the same geometric genus, we infer from the theorem of No. 21 that if $\Gamma_{j}$ is algebraic at the beginning of the deformation it will also be so at the end, that is $\Gamma_{k}$ is then also algebraic, which proves our statement.

Suppose first that no $\Gamma_{k}$ is algebraic. Then any algebraic cycle $\Delta$ of $H_{z}$ must be invariant. For if, when $z$ turns around $c_{i}, \Delta$ is returned to a cycle $\Delta^{\prime}$ of $H_{z}, \Delta-\Delta^{\prime}$ must be algebraic and as $\Delta-\Delta^{\prime} \sim \mu \Gamma_{k}, \mu \Gamma_{k}$ must also be algebraic. Now if integrals of the first kind have no period relatively to $\mu \Gamma_{k}$, they have none relatively to $\Gamma_{k}$, and $\Gamma_{k}$ is also algebraic. We have then a contradiction unless $\mu=0, \Delta \sim \Delta^{\prime}$, which shows that $\Delta$ is invariant. It follows that under our assumptions the complete systems of curves on $H_{z}$ are invariant. If we take then $\rho^{\prime}$ independent continuous systems we may, as shown in No. 30, determine $\rho^{\prime}$ surfaces of $V_{3}$ whose intersections with $H_{z}$ are independent curves of the surface. These $\rho^{\prime}$ surfaces will be themselves independent, hence $\rho \equiv \rho^{\prime}$ and therefore since $\rho \leqq \rho^{\prime}$, finally $\rho=\rho^{\prime}$.

Suppose now that the $(\Gamma)$ 's are all algebraic. What happens then is an immediate corollary of the following proposition, whose proof we shall do no more than outline here*: Any two-cycle of $H_{2}$ is dependent upon the ( $\Gamma$ )'s and the invariant cycles. - The $R_{2}$ invariant cycles are distinct mod. $V_{3}$, hence whatever $\Delta$, two-cycle of $H_{2}$, there is an integer $\lambda$ such that $\lambda \Delta$ is homologous to an invariant cycle mod. $V_{3}$, that is $\Delta^{\prime} \sim \lambda \Delta$-invariant cycle $\sim 0$, mod. $V_{3}$.

\footnotetext{
*A topological proof will be found in the Borel Series monograph.
} 
Now generalizing a discussion of Picard's (see Picard et Simart, Tra it é, vol. 2 , p. 388), pertaining to the periods of certain integrals, and with a ready passage from periods to cycles, which shall be omitted here, it is found that the number of $(\Gamma)$ 's distinct mod. $H_{2}$, (they all bound on $V_{3}$ ), is precisely equal to the maximum number of cycles distinct mod. $H_{z}$, but bounding on $V_{3}$. Hence $\Delta^{\prime}$ depends upon the $(\Gamma)^{\prime} s$, from which our assertion follows.

The application to our problem is immediate: If the $(\Gamma)$ 's are all algebraic, any two-cycle of $H_{z}$ depends upon the invariant and the algebraic cycles.

A proposition of my Annali di matematica paper taken together with a result established in a note of the Rendicontidei Lincei of 1917 leads to this proposition: The number $\rho_{0}=\rho_{0}^{2}$ of $V_{3}$ is equal to the number of non-algebraic two-cycles, or which is the same, to the number of invariant non-algebraic cycles, of $H_{z}$. We also know from Picard's work that the same number for $H_{z}$, which we shall denote by $\rho_{0}^{\prime}$, is equal to its total number of non-algebraic cycles. Hence if $\rho_{0}^{\prime}>\rho_{0}, H_{z}$ possesses some non-invariant non-algebraic cycles, none of the $\left(\Gamma_{k}\right)$ 's are algebraic and $\rho=\rho^{\prime}$. We may therefore state:

THEOREM. If the number of proper integrals of the second kind of a generic hyperplane section exceeds that of $V_{3}$, they have equal numbers $\rho$.

33. Thus for $H$ we have $\sigma^{\prime}=\sigma$, and if $\rho_{0}^{\prime}>\rho_{0}$ also $\rho^{\prime}=\rho$. In these conditions the trace on an arbitrary $H$ of an ordinary base for the surfaces of $V_{3}$ forms a base for the curves of $H$. Let us show that if the first is a minimum base so is the second, this being true even if only effective surfaces or curves are considered.

Indeed let $C_{1}, C_{2}, \cdots, C_{\tau}$ be the surfaces of a mirimum base and $\gamma$ an arbitrary curve of a generic $H_{z}$. As we have seen, the complete continuous systems of curves of $\mathrm{H}_{z}$ are all invariant. Hence the complete continuous system $\left\{\gamma+k H \cdot H_{z}\right\}$ ( $k$ a sufficiently large integer) is invariant and contains $\infty^{q}$ linear systems. One of these systems will be determined by the ( $\beta$ )'s, $\left(\beta_{k}=v_{p-q+k}\right)$, and a curve $\gamma^{\prime}$ of it may be defined rationally on $H_{z}$ by means of an adequate behavior at the points $A_{i}$, for example. The locus of $\gamma^{\prime}$ is an algebraic surface $C$ going, say, $l$ times through the curve at infinity of $H_{z}$, and we have relations such as these: $C=\Sigma \lambda_{i} C_{i}, H=\Sigma \mu_{i} C_{i}$, (on $V_{3}$ ); $C H_{z}=\Sigma \lambda_{i} C_{i} H_{z}, H H_{z}=\Sigma \mu_{i} C_{i} H_{z}$, (on $H_{z}$ ). Hence on $H_{z}$, $\gamma=C H_{z}-(l+k) H H_{z}=\Sigma\left[\lambda_{i}-(l+k) \mu_{i}\right] C_{i} H_{z}$, as was to be proved.

34. The preceding propositions remain true if we replace $|H|$ by $|E|, \infty^{3}$ at least, irreducible, simple, without base points, with irreducible characteristic curve $E^{2}$, such that if the surface $E_{0}$ of the system acquires singularities other than those of the generic $E$, they consist in general in one isolated double point, and that finally the system of the surfaces $E_{0}$ is irreducible. If $|E|$ has a base group but still irreducible variable intersection, we have the following: 
Let $D_{1}, D_{2}, \cdots, D_{k}$ be the finite or infinitesimal curves of the base group forming base for all the surfaces of the group which are not traces of the surfaces of $V_{3}, D_{1}^{\prime}, D_{2}^{\prime}, \cdots, D_{k}^{\prime}$, those forming minimum base in the same conditions. Then if $\rho_{0}^{\prime}>\rho_{0}$ we have $\rho^{\prime}=\rho+k, \sigma^{\prime}=\sigma+k^{\prime}-k$. Moreover, the trace of an ordinary (minimum) base of $V_{3}$ added to the $(D)$ 's (to the $\left(D^{\prime}\right)$ 's) forms an ordinary (minimum) base for $E$.

35. The extension to a $V_{d}, d>3$, is immediate. Since $V_{d}$ and its hyperplane sections have the same two-cycles the restriction $\rho_{0}^{\prime}>\rho_{0}$ in the theorems just proved is now superfluous. We still have $\sigma=\sigma_{1}$.

The case where the two-cycles of $V_{d}$ are all algebraic is especially noteworthy. $H$ need then not be generic in its system but only without new singularities. For, let $H_{0}$ be a special $H$, but with the same singularities as the generic $H, C_{1}, C_{2}, \cdots, C_{\gamma}$ a minimum base for $V_{d}$. The two-cycles $\left(C_{i} H^{d-2}\right)$ form a minimum base for the two-cycles of $H$, and as $H_{0}$ and $H$ are homeomorphic the limiting positions $\left(C_{i} H^{d-3} H_{0}\right)$ of these cycles form a minimum base for the two-cycles of $H_{0}$.

Let now $\gamma$ be any hypersurface of $H_{0}$. We have mod. $H_{0}$

$$
\left(\gamma H^{d-3}\right) \sim \sum \lambda_{i}\left(C_{i} H^{d-3} H_{0}\right) \quad\left(\lambda_{i} \text { integer }\right) .
$$

But the equivalence between the relations $C=D,\left(\mathrm{CH}^{d-2}\right) \sim\left(D H^{d-2}\right)$, proved for $d=2,3$; can easily be shown to hold for any $d$. Applying it to $H_{0}$ we obtain $\gamma=\Sigma \lambda_{i} C_{i} H_{0}$, and therefore the trace of a minimum base of $V_{d}$ on $H_{0}$ is a minimum base for $H_{0}$. Clearly the minimum base could everywhere be replaced by an ordinary one. It follows that $H_{0}$ has the same numbers $\rho, \sigma$ as $V_{d}$.

If $H_{0}$ had special singularities of less than $d-2$ dimensions we could only affirm that its numbers $[\rho],[\sigma]$ are equal to $\rho, \sigma$ respectively. Similar considerations lead to this result: Let $|E|$ be a linear system analogous to that of the same name described in No. 34 for a $V_{3}$ and without base group. If the invariant $\rho_{0}^{d-1}$ of the generic $E$ is greater than that of $V_{d}$, their invariants $\rho_{d-1}$ are equal. Their numbers $\sigma_{d-n}, \rho_{d-i}, i \geqq 2$ are equal without this restriction.

36. Returning to a $V_{3}$, we may remark that there is a case where the condition $\rho_{0}^{\prime}>\rho_{0}$ is certainly verified,-it is when $V_{3}$ possesses a triple integral of the first kind

$$
\iiint \frac{Q(x, y, z, t)}{F_{\imath}} d x d y d z
$$

with periods not all zero. Indeed its periods relatively to cycles within $\mathrm{H}_{z}$ are all zero, as follows from the discussion at the end of the first chapter. Hence there must be periods relatively to finite cycles or special cycles such 
as described in proposition (b) of No. 2. This in turn requires that the double integral of the first kind attached to $H_{z}$,

$$
\iint \frac{Q(x, y, z, t)}{F_{t}^{\prime}} d x d y
$$

be not without periods. But the periods of (12) relatively to algebraic cycles are zero, and those relatively to invariant cycles are rational in $z$, finite everywhere, infinitesimal for $z$ infinite, and therefore also zero. Hence the cycles $\Gamma_{k}$ of No. 32 are not algebraic and $\rho^{\prime}=\rho$ for $|H|$ or for any linear system without base group such as $|E|$, already described. This may also be shown by remarking that (12) is not improper of the second kind and since it is without invariant periods it cannot be derived from an integral of the second kind of $V_{3}$, consequently $\rho_{0}^{\prime}>\rho_{0}$.

Let us now take a $V_{d}$ and call $\Gamma$ a $d$-cycle relatively to which one of its $d$-uple integrals of the first kind has a non-zero period. $\Gamma$ may be the sum of two cycles, the first $\Gamma^{\prime}$ being contained in $H$ and the second $\Gamma^{\prime \prime}$ of the special type described in proposition $(b)$, No. 2. If the period relatively to $\Gamma^{\prime \prime}$ is not zero the reasoning just used for $V_{3}$ applies here and we shall find that $H$ possesses a $(d-1)$-uple integral of the first kind with at least one non-zero period. On the other hand if the period relatively to $\Gamma^{\prime}$ is not zero we may by reasoning as in No. 15, Chapter 1 , show that there is a $(d-2)$-uple integral of the first kind of $\mathrm{H}^{2}$ having at least one non-zero period. Continuing this we shall come either to an $H^{d-3}$ or to an $H^{d-2}$ with integrals of the first kind of multiplicity $d-3$ or $d-2$ having at least one non-zero period. In the first case it follows at once that $H^{d-2}$ has the same number $\rho$ as $H^{d-3}$ and therefore as $V_{d}$, its bases being the traces of those of $V_{d}$. In the second case the reasoning applied for $V_{3}$ holds as between $H^{d-3}$ and $H^{d-2}$, leading to the identical conclusion.

As an application, a generic $V_{2}$, complete intersection of $d-2$ hypersurfaces in a $V_{d}$, each generic in an adequate linear system, has for bases the traces of those of $V_{d}$, provided there is a $d$-uple integral of the first kind of $V_{d}$ with periods not all zero.

The most interesting case is that of an Abelian variety of genus $p$ and rank one, for if $u_{1}, u_{2}, \cdots, u_{p}$ are the variables occurring in its parametric representation $\int \mathcal{S} \cdots \mathcal{S} d u_{1} d u_{2} \cdots d u_{p}$ is an integral of the first kind with at least one non-zero period.

37. For a $V_{d}, d>2$, contrary to what happens for an algebraic surface, the number of two-cycles without periods for the integrals of the first kind is only a maximum of $\rho$. At all events we have succeeded in proving that these two numbers are equal only for Abelian varieties, as we shall see in the second part, and for a certain type of $V_{3}$, which may be defined thus: let $P_{a}, P_{b}$ be 
respectively the arithmetic and geometric genus of a $V_{3}$. If $P_{0}-P_{a}+q=0$, all the two-cycles are algebraic, and therefore $\rho$ reaches its maximum. Indeed as shown by Severi, in this case the system adjoint to $|H|$ cuts out the complete canonical system on $H$. Hence every double integral of the first kind of $H_{z}$ is a linear combination of those of type $\int \mathcal{S}\left(1 / F_{t}^{\prime}\right) Q(x, y, z, t) d x d y$, where $Q$ is adjoint of order $m-4$, whose periods relatively to invariant cycles all vanish. It follows then that all these cycles are algebraic cycles of $H_{z}$ and, being invariant, they are also algebraic cycles of $V_{3}$. In this case $\rho_{0}=R_{2}-\rho=0$, hence $\rho=R_{2}$.

\section{$\S 4$. Some applications}

38. Complete intersections. Let $V_{2}$ be a non-singular algebraic surface, complete intersection of $(r-2)$ varieties in an $S_{r}$. I say that $V_{2}$ has no double integrals of the first kind without periods. For let $F(x, y, z)=0$ be the equation of its projection $V_{2}^{\prime}$ in an $S_{3}$. It is sufficient to show that there can be no relation $A F_{x}^{\prime}+B F_{y}^{\prime}+C F_{z}^{\prime} \equiv-D F$, where $A, B, C$ are adjoint of order $m-2$. For according to this relation the $N$ contacts of the planes $y=C t$, tangent to $V_{2}^{\prime}$, must be on $B=0$. Now if $|K|$ designates the canonical system and $|H|$ the complete system of the plane sections, the adjoint surfaces of order $m-2$ cut out on $V_{2}^{\prime}$ the system $|K+2 H|$. They intersect the first polar of an arbitrary point of $S_{3}$ in. a number of points exterior to the double curve, at most equal to*

$$
[(K+2 H)(K+3 H)]=\left[K^{2}\right]+5[K H]+6\left[H^{2}\right],
$$

and hence it is only necessary to prove that this number is $<N$. Let $m_{1}, m_{2}$, $\cdots, m_{r-2}$, be the orders of the varieties of $S_{r}$ of which $V_{2}$ is the intersection. Then $m=m_{1} m_{2} \cdots m_{r-2}$, and the adjoint system is cut out on $V_{2}$ by the varieties of order $n=\Sigma m_{i}-r-1$; therefore $\left[K^{2}\right]=m n^{2},[K H]=m n$, $\left[H^{2}\right]=m$. Hence the number considered above is equal to $m n^{2}+5 m n+6 m$. To find the value of $N$ we observe that in $S_{r}$ the hyperplanes of a linear net which are tangent to $V_{2}$ touch the surface at the points where it is intersected by a variety of order $n+3$, hence $N=m(n+3)^{2}>m n^{2}+5 m n+6 m$, which proves our assertion.

This still applies when all the $(m)$ 's are equal to unity except for one which is $>3$, that is, when we deal with a non-singular algebraic surface of order $>3$ in ordinary space.

39. Now a non-singular $V_{r-1}$ in $S_{r}, r>3$, has for minimum base the trace of a minimum base of $S_{r}$, that is, a hyperplane section. Its index $R_{2}=1$ as it is for $S_{r}$ and its two-cycles are all algebraic. Its complete systems of hyper-

* It is in general less than this number by a certain multiple of the number of pinch points owing to the peculiar behavior of the first polars at these points. 
surfaces are all linear. Here then, equivalence à la Severi between two hypersurfaces implies that they are contained in the same linear system, and as they are all multiples of a hyperplane section, $V_{r-1}$ contains only hypersurfaces which are complete intersections. The same reasoning holds for its intersection with another non-singular $V_{r-1}$ of $S_{r}$, provided that this intersection is nonsingular and also that $r>4$, and so on. We thus see that $a$ non-singular $V_{d}, d>2$, complete intersection in an $S_{r}$, contains only hypersurfaces that are themselves complete intersections.

Let now $d=2$. Any $V_{3}$, complete intersection and non-singular, passing through $V_{2}$, possesses no proper double integrals of the second kind since its $R_{2}=\rho$, whereas $V_{2}$ certainly possesses such an integral if its order is $>1$ and no space of less than four dimensions can be passed through it, or, as this is usually stated, if it is normal in an $S_{r}, r>3$. Hence a non-singular $V_{2}$, complete intersection and normal in an $S_{r}, r>3$, contains in general only curves which are complete intersections. This is true also for a non-singular surface of order $>3$ in ordinary space.* For ordinary space this theorem has been stated and proved many years ago by Noether, but his proof based on enumeration of constants has long been considered unsatisfactory. The above is believed to be the first complete proof ever given of this important proposition.

As shown previously, even limiting ourselves to ordinary space, we can go farther. Let $|E|$ be a linear system, simple, irreducible, $\infty^{3}$ at least, with a base group composed of $k$ curves independent on $E$ and such that no point is multiple for any $E$ through it. The generic $E$ contains no other curves than those cut out by surfaces passing through curves of the base group, and for it $\rho=k+1, \sigma=1$.

40. The part of our results relating to a $V_{r-1}$ in $S_{r}, r>3$, has already been obtained by Severi. The cubic variety in $S_{4}$, (Segre variety), has been treated at length by $\mathrm{G}$. Fano. It furnishes a very interesting illustration. Indeed it may be,verified that when there are less than six double points the variety belongs to a linear system, $\infty^{4}$ at least, and with the properties described more than once, hence it contains only complete intersections. For the hyperplane sections whose geometric genus is zero, $\rho=7$. Intersections with hypersurfaces of order $\equiv 2$ contain in general only complete intersections.

41. Double plane. Let $z^{2}=f(x, y)$ be the equation of a double plane. One may always transform it birationally so that the branch curve be of even order $2 m$, without multiple components and with multiple points all of even order and with distinct tangents. Any double integral of the first kind is of

* During the final preparation of this memoir the author's attention was attracted by an incomplete proof of these theorems due to G. Fano, ( T o r in o A t ti, vol. 41 (1909), pp. 415-430) which has some points in common with the one given here. Fano admits that a double integral of the first kind cannot be without periods, and this can scarcely be considered as axiomatic. 
the form $\mathcal{S} \mathcal{S}[f(x, y)]^{-t} Q(x, y, t) d x d y$, where $Q$ is a polynomial of order $m-3$ and has an $(i-1)$-uple point at a $2 i$-uple point of $f(x, y)$, (Enriques). If the periods are zero one may show that equations (8) now become $A_{x}^{\prime}+B_{y}^{\prime}+C_{t}^{\prime} \equiv Q(x, y, t), A f_{x}^{\prime}+B f_{y}^{\prime}+C f_{t}^{\prime} \equiv 0$, where $A, B, C$ are of order $m-1$ and behave like $Q$ at the multiple points of $f$. Here again the points of intersection, other than the multiple points of $f=0$, of the curves $f_{x}^{\prime}=0, f_{y}^{\prime}=0$, must be on $C=0$. Hence $C=0$ and $f_{x}^{\prime}=0$ have in common at least

$$
\begin{aligned}
(2 m-1)^{2}-\sum(2 i-1)^{2}+\sum(2 i-1)(i-1) & =(2 m-1)-\sum i(2 i-1)
\end{aligned}
$$

points, the summation being extended to all the multiple points. To show that our double integral cannot be deprived of periods it is sufficient to show that this number exceeds $(m-1)(2 m-1)$, which, since $f_{x}^{\prime}=0$ may be assumed irreducible, will lead to an impossibility. But the inequality to be proved reduces at once to $m(2 m-1)>\Sigma i(2 i-1)$ which is actually verified, for it may be obtained by expressing that the number of isolated double points equivalent to the multiple points of $f(x, y)$ does not exceed its value $m(2 m-1)$ when $f$ is a product of $2 m$ linear factors. Thus a double plane has no double integral of the first kind without periods.

42. When does a double plane belong to a linear system with the properties described in No. 34? I say that this will certainly occur if $f(x, y)$ is divisible by a factor $\phi(x, y)$ such that the curve $\phi(x, y)=0$ be generic in a linear system $|\gamma|, \infty^{2}$ at least, not composed with the curves of a pencil, and such that there is no point not a base point multiple for any $\gamma$ through it. For let $f \equiv \phi \cdot \psi$, and $\phi+k \phi_{1}=0$ the equation of a generic pencil of $|\gamma|$. First we verify that the variable curve of intersection of the two surfaces $z^{2}=\phi \psi$, $z^{2}=\left(\phi+k \phi_{1}\right) \psi$, is irreducible. Indeed if it were not the two surfaces would have the same tangent planes at the points $z=\phi=\phi_{1}=0$ which are not base points for $|\gamma|$, and the curves $\phi=\phi_{1}=0$ would be tangent at those points, which is not the case. Next the multiple points of the double plane are the multiple points of $f=0$, and a fixed $(2 m-2)$-uple base point at infinity, hence there is no point other than a base point multiple for all the double planes having for branch curve $\psi=0$ associated with a curve $\gamma$, and we can apply our general theorems to them.

43. To find the minimum base we must first determine whether the components of the branch curve are independent curves of the double plane. Let $f \equiv \phi_{1} \cdot \phi_{2} \cdots \phi_{h}$, where the $(\phi)$ 's are irreducible polynomials prime to each other, and let $C_{i}$ be the curve $\phi_{i}=0$ of the double plane. Denoting by $|H|,\left\{H_{y}\right\}$ the same elements as usual, we consider $m-1$ independent integrals of the first kind $u_{1}, u_{2}, \cdots, u_{m-1}$ of $H_{y}$ and observe that since in 
the case here considered the double plane is regular (Castelnuovo-Enriques), a relation $\Sigma t_{i} C_{i}=0$ would necessarily lead to another

$$
\sum_{i, j} t_{i} \int_{(x, s)}^{M_{i j}} d u_{s}=0 \quad(s=1,2, \cdots, m-1),
$$

where the points $M_{i j}$ are the points of the group $C_{i} H_{y}$, and the lower limit of integration is arbitrary on $H_{y}$. Now $H_{y}$ is hyperelliptic of genus $m-1$, and, as far as the integrals at the left are concerned, one may be made zero, while their sum vanishes. We also know that by combining them linearly with suitable integral coefficients we obtain $2 m-2$ independent periods of $u_{s}$. Hence the only relation of the type considered corresponds to

$$
t_{1}=t_{2}=\cdots=t_{h}=t,
$$

and as we have $t \Sigma C_{i}=t H \neq 0$, the (C)'s are actually independent.

We conclude from this that if $p_{o}>0$ and one of the $(C)$ 's is variable in a suitable linear system, the minimum base is composed of: (a) The vicinity of the singular point at infinity on the $z$ axis which counts for $h$ curves. (b) The vicinities of the multiple points of the branch curve which will count for, say, $k$ curves. The value of $k$ will depend upon the nature of the singular points, etc. In the simplest case where the curve has only ordinary double points $k$ will be equal to their number.

(c) The $h$ components of the branch curve. We have therefore $\rho=2 h+k$, $\sigma=1,[\rho]=h$. Every curve of the double plane is cut out by a surface which goes through several of the $(C)$ 's and through no other curve of the double plane.

As to $\rho_{0}$, it may be computed thus: If $N$ is the class of the branch curve, $d$ the equivalence in double points of its multiple points we find by means of $\left\{H_{y}\right\},\left[R_{2}^{\prime}\right]=N-6 m+5+d$. Therefore

$$
\rho_{0}=\left[R_{2}^{\prime}\right]-[\rho]+1=N-6 m+6+d-h .
$$

This discussion can be applied to derive some of Picard's results for double planes as well as some due to Enriques for surfaces of linear genus $p_{1}=1$. Let us take for example the surface $z^{2}=a(y) x^{3}+b(y) x^{2}+c(y) x+d(y)$, where $a, b, c, d$, are polynomials, a surface considered by Picard.* If these polynomials are arbitrary and prime to each other the double plane contains only complete intersections and this relation cannot be satisfied by substituting for $z$ and $x$ rational functions of $y$.

Enriques showed that the surfaces named after him are reducible to double planes, the determination of their minimum base as he gave it is then immediate. $t$ These double planes contain also only complete intersections.

* Picard-Simart, Traité des fonctions algébriques de deux variables, vol. 2, p. 268.

t Rendiconti de i Lincei, ser. 2, vol. 23 (1914), pp. 291-297. 


\section{$\S 5$. Varieties representing the groups of points of other varieties}

44. We insert here a short discussion of these varieties that will be found useful in some applications. They present also the added interest that we can form very readily varieties of this type having quite arbitrary torsion indices.

Let then $V^{1}, V^{2}, \cdots, V^{n}$ be algebraic varieties with ordinary singularities, $d_{i}$ the dimensionality of $V^{i}, V_{d}$ a variety representing without exception the groups of $n$ points of which each is on a $V^{i}$, so that $d=\Sigma d_{i}$.

By generalizing somewhat the reasoning at the beginning of this paper, it may be shown that all the $i$-cycles are sums of those obtained by associating an $i_{1}$-cycle of $V^{1}$, an $i_{2}$-cycle of $V^{2}, \cdots\left(i_{1}+i_{2}+\cdots=i\right)$ in the same manner as $V^{1}, V^{2}, \cdots$. Let then $R_{i}^{h}, R_{i}$ and $\sigma_{i}^{h}, \sigma_{i}$ be the $i$ th indices of connectivity and torsion of $V^{h}$ and $V_{d}$ respectively. We shall have $R_{i}=$ $\Sigma R_{i_{1}}^{1} R_{i_{2}}^{2} \cdots R_{i_{n}}^{n}$, the summation being extended to all the partitions of the ( $i$ )'s such that $i=i_{1}+i_{2}+\cdots+i_{n}, i_{h} \leqq 2 d_{h}$, and with the convention that $R_{2 d_{n}}^{h}=R_{0}^{h}=1$.

As to the $(\sigma)$ 's suffice to state that $\sigma_{1}=\sigma_{1}^{1} \sigma_{1}^{2} \cdots \sigma_{1}^{h}$. Now Godeaux has shown that there are algebraic surfaces whose invariant $\sigma=\sigma_{1}$ is any odd prime, and an example of $\sigma=2$ has been given by Severi. This shows that there are algebraic varieties for which $\sigma$ has any value whatever. We shall have occasion to consider in the second part Abelian varieties of rank $>1$ whose invariant $\sigma$ is arbitrarily assigned and in fact equal to the rank. Suitable surfaces contained in these varieties will have the same value of $\sigma$, i.e., equal to an arbitrary integer.

Remark. All the varieties so far known, including those to be constructed in the second part, for which $\sigma>1$, represent involutions on varieties whose $\sigma=1$. The question presents itself - are there any others? For the present it must remain unanswered.

45. Let us now pass to the determination of $\rho$. Assume first $n=2$. $V_{d}$ contains a continuous system $\infty^{d_{1}}, \Sigma_{1}$, of varieties identical with $V^{2}$ and another $\infty^{d_{2}}, \Sigma_{2}$, of varieties identical with $V^{1}$. A hypersurface of $V_{d}$ representing the pairs of points of $V^{2}$ and a hypersurface of $V^{1}$ will be called fundamental for $V^{1}$. Similarly there will be hypersurfaces fundamental for $V^{2}$. The hypersurfaces fundamental for $V_{i}$ possess an ordinary (minimum) base composed of $\rho_{i},\left(\rho_{i}+\sigma_{i}-1\right)$, hypersurfaces. Assume that $E_{i}$ is fundamental for $V^{i}$. Then certainly there can be no relation $E_{1}=E_{2}$ since the homology $\left(E_{1}\right) \sim\left(E_{2}\right)$ is not verified. Hence the hypersurfaces of the two fundamental sets are not related.

To find the number of hypersurfaces independent of those of the fundamental sets, I say that if $d_{1} \equiv 3, V^{1}$ may be replaced by a $V_{d_{1}-1}$ having the 
same Picard variety $W_{1}$ as $V^{1}$. For let $\left\{C_{\mu}\right\}$ be an arbitrary linear pencil of hypersurfaces fundamental for $V_{1}$. If $A, B$ are two hypersurfaces such that $A C_{\mu}=B C_{\mu}$ on any $C_{\mu}$, then $A-B$ is fundamental for $V^{1}$. This can be shown in a manner very similar to that used to prove the analogous theorem for any $V_{3}$ and the pencil $\left\{H_{2}\right\}$. If $\left\{C_{\mu}\right\}$ were generic in a suitable linear system we would have $A=B$, but here we may only conclude that $A-B$ is a multiple of $C_{\mu}$. It follows then that as far as we are concerned $V^{1}$ may be replaced by $C_{\mu}$ whose Picard variety will be $W_{1}$, if $d_{1} \equiv 3$.

We propose to show next that just as in the case $d_{1}=d_{2}=1$ already investigated by Severi,* the number of hypersurfaces independent of those of the two fundamental sets is equal to Scorza's simultaneous index $\lambda_{12}$ of the Picard varieties $W_{1}, W_{2}$ of $V_{1}$ and $V_{2}$. This index will be defined in the second part (No. 47). We may then assume $d_{1} \leqq 2, d_{2} \leqq 2, d_{1}+d_{2}>2$. One of the $(d)$ 's, say $d_{1}$, will have the value two.

Let $A$ be an arbitrary hypersurface of $V_{d},\left\{C_{1}\right\}$ a complete continuous system of curves of $V^{1}$ containing $\infty^{q_{1}}$ linear systems, ( $q_{i}$ irregularity of $\left.V^{i}\right), C_{1}^{1}$ the fundamental hypersurface determined by $C_{1}$. To the points $C_{1}^{1} A$ may correspond either all the points' of $V^{2}$ or only a $V_{d_{2}-1}$ of $V^{2}$. The first case may be rejected at once for then there corresponds to the points in question only a finite number of points on $C_{1}$, and to $A$ only a curve on $V^{1}$; hence $A$ is fundamental. In the second case, to which we may limit ourselves then, there corresponds to $C_{1}^{1} A$ a certain algebraic curve $C_{2}$ on $V^{2}$ if $d_{2}=2$, or a group of points if $d_{2}=1$. But $C_{1}$ determines a point of $W_{1}$ and $C_{2}$ one of $W_{2}$, for by adding if necessary a suitably chosen fundamental hypersurface to $A$, we may arrange matters so that $C_{2}$ belongs to a continuous system containing $\infty^{q_{2}}$ linear systems. We have here an algebraic correspondence between the points of $W_{1}$ and $W_{2}$ and the theory of Hurwitz-Severi may be extended at once, leading to the announced result. The extension to $n>2$ is immediate. It is sufficient to consider $V_{d}$ as corresponding to the group of two points one of which is on $V^{1}$ and the other on the $V_{d-d_{1}}$, obtained by associating $V^{2}, \cdots, V^{n}$. We shall have finally $\rho=\Sigma \rho^{(i)}+\Sigma \lambda_{i k}$, where $\rho^{(i)}$ is the number $\rho$ of $V^{i}$ and $\lambda_{i k}$ the simultaneous index of the-Picard varieties of $V^{i}$ and $V^{k}$.

*Torino M e mori e, ser. 2, vol. 54 (1903), pp. 1-49.

Trans. Am. Math. Soc. 24 


\section{PART II}

\section{ABELIAN VARIETIES}

\section{Chapter I. General Properties}

\section{$\S 1$. A summary of certain fundamental theorems and definitions}

46. An Abelian variety of genus $p, V_{p}$, is a variety whose non-homogeneous point coördinates are equal to $2 p$-ply periodic meromorphic functions of $p$ arguments $u_{1}, u_{2}, \cdots, u_{p}$, or whose homogeneous point coördinates are proportional to theta's of the same order and continuous characteristic. The variety is algebraic (Weierstrass) and of dimensionality $p$. When the periods are those of a set of independent integrals of the first kind of a curve of genus $p, V_{p}$ is called a Jacobi variety.

An array with $p$ rows and $2 p$ columns

$$
\Omega \equiv\left\|\omega_{j \mu}\right\| \quad(j=1,2, \cdots, p ; \mu=1,2, \cdots, 2 p)
$$

is the period matrix of a $V_{p}$ provided that:

(a) There exists an alternate bilinear form with rational coefficients

$$
\sum_{\mu, \nu=1}^{2 p} c_{\mu \nu} x_{\mu} y_{\nu}, \quad c_{\mu \nu}=-c_{\nu \mu},
$$

vanishing identically when the $(x)$ 's and the $(y)$ 's are replaced by the elements of any two rows of $\Omega$.

(b) If we set $\xi_{\mu}+i \eta_{\mu}=\Sigma_{(j)} \lambda_{j} \omega_{j_{\mu}}$, then, for all non-zero values of the $(\lambda)$ 's, $\Sigma c_{\mu \nu} \xi_{\mu} \eta_{\nu}>0$. According to Scorza $(b)$ is equivalent to this: Denoting by $\bar{x}$ the conjugate of any number $x$, the Hermitian form in the $(\lambda)$ 's,

$$
\sum A_{j k} \lambda_{j} \lambda_{k}, \quad A_{j k}=-\frac{1}{2 i} \sum c_{\mu \nu} \omega_{j \mu} \bar{\omega}_{k \nu},
$$

must be definite positive. When these conditions are satisfied $\Omega$ is called by the same author a Riemann matrix.

47. We propose now to recall some concepts and definitions incipient in. the works of various authors, but formally introduced and only fully developed in recent writings of Scorza and also Rosati.* The nomenclature which we shall use is Scorza's.

The alternate form ( 1 ) is called a Riemann form of $\Omega$. If the condition (b) is satisfied for that form, it is said to be a principal form of the matrix. There

* See Scorza's memoir in the Rendiconti di Palermo, vol. 41 (1916), for numerous bibliographical indications. The very interesting method which dominates his and Rosati's investigations had been used previously without their being apparently aware of it by Cotty in his Paris thesis (1912) for the case $p=2$. Cotty attributes the idea to Humbert. We shall have opportunity to apply it on several occasions. 
may be several independent forms alternate of $\Omega$, and if $1+k$ is their number, $k$ is called the index of singularity. If $k \neq 0$ the matrix is said to be singular.

There may be $1+h$ bilinear forms with rational coefficients

$$
\sum_{\mu, \nu=1}^{2 p} c_{\mu \nu} x_{\mu} y_{\nu}
$$

vanishing identically when the ( $x$ )'s and the $(y)$ 's are replaced by the elements of any two rows of $\Omega$. The integer $h$ is called the index of multiplication, and we have $h \geqq k$.

Finally, given two Riemann matrices $\Omega, \Omega^{\prime}$, if there exist $\lambda$ bilinear forms with rational coefficients

$$
\begin{array}{r}
\sum c_{\mu \nu} x_{\mu} y_{\nu} \quad\left(\mu=1,2, \cdots, 2 p ; \nu=1,2, \cdots, 2 p^{\prime} ;\right. \\
\left.p, p^{\prime} \text { genera of } \Omega, \Omega^{\prime}\right)
\end{array}
$$

which vanish identically when the $(x)$ 's are replaced by the elements of a row of $\Omega$ and the $(y)$ 's by those of a row of $\Omega^{\prime}, \lambda$ is called the index of simultaneity of the two matrices.

The forms of types (1), (3), (4), generate moduli and admit bases. The corresponding numbers $\sigma$ are all equal to one.

Two matrices $\Omega, \Omega^{\prime}$ are isomorphic if we can pass from one to the other by a linear transformation with arbitrary coefficients applied to the rows and by a linear transformation with rational coefficients applied to the columns. If this last transformation is of determinant one and integral coefficients (unimodular), $\Omega, \Omega^{\prime}$ are equivalent. In all cases the determinants of the two transformations must not be zero. When two matrices are isomorphic, the most general Abelian varieties which correspond to them in the sense defined below, are in algebraic correspondence with each other. When the matrices are equivalent, their Abelian varieties can be transformed birationally into each other.

A Riemann matrix $\Omega$ is said to be impure if it is isomorphic to one of type

$$
\left\|\begin{array}{cl}
\Omega_{1}, & 0 \\
0, & \Omega_{2}
\end{array}\right\|
$$

where $\Omega_{1}, \Omega_{2}$, are Riemann matrices of genus $p$ and the ciphers represent matrices with elements all zero. If $\Omega$ is not impure, it is said to be pure. When $\Omega$ is impure the field of Abelian functions which belongs to it contains functions of genus $<p$, (Poincaré). Finally, in the same case, an Abelian variety belonging to $\Omega$ represents the pairs of points, one of which is on an Abelian variety belonging to $\Omega_{1}$ and the other on one belonging to $\Omega_{2}$.

The results at the end of the preceding part allow us to replace the determination of the base invariants of an impure variety by that of the invariants of varieties of genus $<p$. We shall return to this point later.

Remark. All the properties which we have recalled here are invariant in regard to isomorphism. 
48. The matrix $\Omega$ is equivalent to a matrix in the so-called canonical form

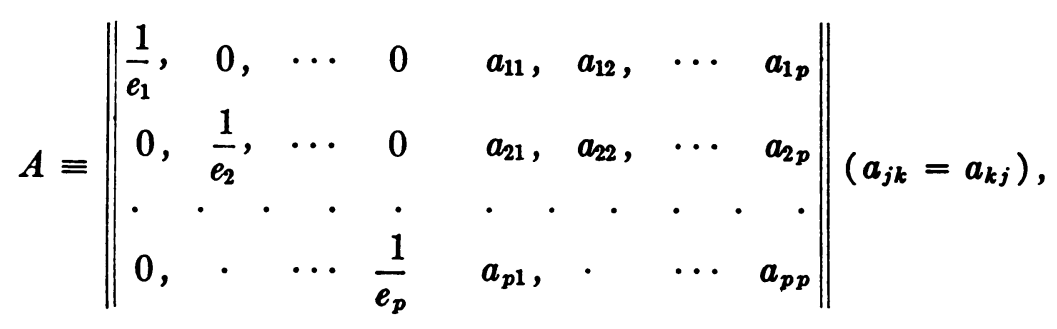

where the integers $e_{\mu}$ are the elementary divisors of (1), assumed now with integral coefficients. The matrix has a principal form $\Sigma_{\mu=1}^{p} e_{\mu}\left(x_{\mu} y_{p+\mu}-x_{p+\mu} y_{\mu}\right)$ derived from (1) up to an integral factor, and condition (b) gives now this: If $a_{j k}=a_{j k}^{\prime}+i a_{j k}^{\prime \prime}$, the quadratic form $\Sigma a_{j k}^{\prime \prime} x_{j} x_{k}$ must be definite pnsitive, or, which is the same, $\Sigma a_{j k}^{\prime \prime} x_{j} x_{k}=1$ must be a real generalized ellipsoid. The arbitrariness of the ( $\left.a^{\prime \prime}\right)$ 's shows that in general the above alternate form is unique - that is, that for an arbitrary Riemann matrix $k=0$.

Let us indicate a certain property of the Hermitian form (2), interesting especially in some applications. Designate by $C$ an arbitrary alternate Riemann form of $\Omega$, for example (1), then apply a transformation $B$ of nonzero determinant defined by

$$
\begin{aligned}
\omega_{j \mu}^{\prime}=\sum_{\nu=1}^{2 p} b_{\mu \nu} \omega_{j \nu} \\
\quad(\mu=1, \dot{2}, \cdots, 2 p ; j=1,2, \cdots, p) .
\end{aligned}
$$

To the matrix of the $\left(\omega^{\prime}\right)$ 's will correspond an alternate form $C^{\prime}$ defined in the notation of Frobenius by $C^{\prime}=\bar{B}^{-1} C B^{-1}$, where $\bar{B}$ is the transposed of $B$.

Consider now the transformation $D$ defined by the matrix

$$
\left\|\begin{array}{lccc}
\omega_{11}, & \omega_{12}, & \cdots & \omega_{1,2 p} \\
\omega_{p 1}, & \cdot & \cdot & \cdot \\
\bar{\omega}_{11}, & \cdot & \cdots & \bar{\omega}_{p, 2 p} \\
\dot{\omega}_{1,2 p}, & \cdot & \cdot & \dot{\omega}_{p 1} \\
\omega_{p 1} & \cdot & \cdots & \bar{\omega}_{p, 2}
\end{array}\right\|
$$

whose determinant as we know is not zero. Applying finally to the $(\omega)$ 's the transformation $\bar{D}^{-1}$, the form $C$ will be replaced by a form $C^{\prime \prime}$ of same genus $q \leqq p$ as $C$, that is, depending essentially upon the same number of linearly distinct variables, ( $2 q$ for the $(x)$ 's, $2 q$ for the $(y)$ 's), as $C$. But owing to the relations $\Sigma c_{\mu \nu} \omega_{j \mu} \omega_{k \nu}=\Sigma c_{\mu \nu} \bar{\omega}_{j_{\mu}} \bar{\omega}_{k v}=0$, we find that the matrix of the coefficients of $C^{\prime \prime}$ is equal to the product of $2^{2 p}$ by

$$
\left.\| \begin{array}{cc}
0, & A
\end{array}\right)
$$


where $A$ is the matrix ||$A_{j k}||(j, k=1,2, \cdots, p)$. If we call genus of a Hermitian form the number of essentially distinct variables upon which it depends, we see that the genus of an alternate Riemann form of $\Omega^{\prime}$ is equal to that of the corresponding Hermitian form.

50. Rank of Abelian Varieties. Let us transform $\Omega$ into a canonical matrix and, using the same notations as in Krazer's Lehrbuch der Thetafunctionen, consider the equations

$$
\tau x_{j}=\theta_{k}^{j}\left[\begin{array}{l}
q \\
h
\end{array}\right](u) \quad(j=1,2, \cdots, 8>p),
$$

where the $(\theta)$ 's are linearly independent theta-functions of sufficiently high order $k$ belonging to $A$. The $(x)$ 's are point coördinates of an Abelian variety belonging to $A$, or if we wish to $\Omega$. Let $v_{1}, v_{2}, \cdots, v_{p}$ be the variables corresponding to $\Omega$. To every system of values of the $(v)$ 's corresponds one and only one of the $(u)$ 's modulo the periods of $A$; and conversely to every system of values of the $(u)$ 's corresponds one and only one of the $(v)$ 's modulo the periods of $\Omega$. The relations $v_{h}=\Sigma_{\mu=1}^{2 p} t_{\mu} \omega_{h_{\mu}}(h=1,2, \cdots, p)$, where the $(t)$ 's are real variables such that $0 \leqq t_{\mu}<1$, define for each system of values of the $(u)$ 's, or the (v)'s, a unique point of an $S_{2 p}$ of which the $(t)$ 's are considered as non-homogeneous point coördinates. The point-set thus obtained fills up a generalized cube $U_{2 p}$, at least if we assume, as we may, that the axes of the $(t)$ 's in $S_{2 p}$ are rectangular. We shall make frequent use of this generalized cube. We could have defined it in terms of the $(u)$ 's but for certain applications it is more advantageous to relate directly $\Omega$, and $U_{2 p}$.

Now, to every point of $U_{2 p}$ corresponds one and only one of $V_{p}$. However, it may be that to any point of $V_{p}$ there correspond more than one of $U_{2 p}$. Let $r$ be the number of these points. It has been called by Enriques and Severi the rank of $V_{p}$.

Let us show that the most general Abelian variety belonging to $V_{p}$ is of rank one. If $V_{p}$ is such a variety it will be possible to express every periodic function belonging to $\Omega$ as a rational function of the $\left(\theta_{k}\right)$ 's. Assume this condition fulfilled and let $(u),\left(u^{\prime}\right)$ be two points of $U_{2 p}$ which correspond to the same point of $V_{p}$. We shall have

$$
\frac{\theta_{k}^{1}(u)}{\theta_{k}^{1}\left(u^{\prime}\right)}=\frac{\theta_{k}^{2}(u)}{\theta_{k}^{2}\left(u^{\prime}\right)}=\cdots=\frac{\theta_{k}^{\prime}(u)}{\theta_{k}^{2}\left(u^{\prime}\right)} .
$$

But the periodic functions are in fact rational in the ratios of $\left(\theta_{k}\right)$ 's, hence they take the same values at $(u)$ and $\left(u^{\prime}\right)$. This will be the case in particular for the ratios of the functions $\theta_{k}(u-e)$, since they are periodic. It follows that the above system will still be satisfied when the $\left(u\right.$ )'s and the $\left(u^{\prime}\right)$ 's are replaced by the quantities $u_{i}-e_{i}, u^{\prime}-e_{i}$ respectively, the $(e)$ 's being any 
constants. Hence, if we replace $u_{i}$ by $u_{i}+d u_{i}$ we must, in virtue of the functional relationship thus established, replace $u_{i}^{\prime}$ by $u_{i}^{\prime}+d u_{i}$. Otherwise stated

$$
\begin{aligned}
d u_{i}^{\prime} & =\frac{\partial u_{i}^{\prime}}{\partial u_{1}} d u_{1}+\frac{\partial u_{i}^{\prime}}{\partial u_{2}} d u_{2}+\cdots+\frac{\partial u_{i}^{\prime}}{\partial u_{p}} d u_{p}=d u_{i} \\
\therefore \frac{\partial u_{i}^{\prime}}{\partial u_{k}} & =0, \quad i \neq k ; \quad \frac{\partial u_{i}^{\prime}}{\partial u_{i}}=1 ; \quad u_{i}^{\prime}=u_{i}+\omega_{i} .
\end{aligned}
$$

The $(\omega)$ 's form a system of constants which may be added to the $(u)$ 's without changing the values of the ratios $\theta_{k}^{j}\left[\begin{array}{l}g \\ h\end{array}\right](u) /\left\{\theta_{k}^{l}\left[\begin{array}{l}g \\ h\end{array}\right](u)\right\}$. They are therefore simultaneous periods of these periodic functions, and the points $(u)$, $\left(u^{\prime}\right)$ coincide, which means that $r=1$.

Thus to every Riemann matrix, corresponds a class of Abeliah varieties of rank one and they are obviously birationally equivalent.

51. Let us show that amongst the varieties of rank one, there exists always one without singularities situated in a suitable space and in point to point correspondence without exception with $U_{2 p}$.

Let us start again from the representation (5) where we shall assume the $\left(\theta_{k}\right)$ 's such that any other $\theta$ of the same order and characteristic is a linear combination of them. Two circumstances may prevent that $V_{p}$ as represented by equations (5) satisfy the desired conditions.

(a) All the $\left(\theta_{k}\right)$ 's vanish for at least one point, $(u)$.

(b) There exists at least one point, $(u)$, for which the hyperplanes of $S_{\infty-1}$ represented by

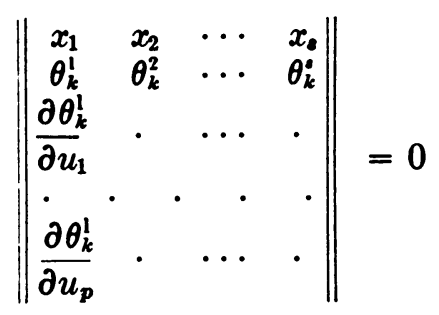

have in common a space of more than $p$ dimensions or, if we prefer, there exists at least one point, $(u)$, for which the array

$$
\left\|\begin{array}{cccc}
\theta_{k}^{1} & \theta_{k}^{2} & \cdots & \theta_{k}^{a} \\
\frac{\partial \theta_{k}^{1}}{\partial u_{1}} & \cdot & \cdots & \cdot \\
\cdot & \cdot & \cdot & \cdot \\
\frac{\partial \theta_{k}^{1}}{\partial u_{p}} & \cdot & \cdots & \cdot
\end{array}\right\|
$$

is of rank $<p+1$.

Let $W$ be the manifold of $U_{2 p}$, or, if we please, the algebraic sub-variety 
of $V_{p}$ for which either circumstance presents itself. The question is to show that for a suitable choice of the representation (5) there is no manifold of this nature. Let us remark first that among the functions $\theta_{n k}\left[\begin{array}{l}g \\ h\end{array}\right]$ are found the $\left(\theta_{k}\left[\left(\begin{array}{l}(g+a) / n \\ \left(\begin{array}{l}n \\ b\end{array}\right) / n\end{array}\right]\right)^{n}(a, b\right.$, integers). Hence if, whatever the representation (5), $W$ contained points of type $(a)$, the functions $\theta_{k}\left[\left(\begin{array}{l}(g+a) / n \\ (h+b) / n\end{array}\right](u)\right.$, would all have a point in common. Since for $n$ great enough there is a characteristic $\left[\left(\begin{array}{l}g+a \\ h+b\end{array}\right) / n\right]$ differing as little as we please from any given one, by passing to the limit, we could conclude that the $(\theta)$ 's of a given order all vanish at a point $\left(u_{0}\right)$ of $V_{p}$. This is equivalent to stating that for any $\theta_{k}$,

$$
\theta_{k}\left(u_{1}^{0}-e_{1}, u_{2}^{0}-e_{2}, \cdots, u_{p}^{0}-e_{p}\right)=0,
$$

whatever the $(e)$ 's, or else $\theta_{k} \equiv 0$.

Thus, when the characteristic is arbitrary, case $(a)$ will certainly not present itself.

Let us show now that case (b) may as well be avoided. Consider the representation

$$
\tau x_{i}=\theta_{n k}^{j}\left[\begin{array}{l}
g \\
h
\end{array}\right](u) \quad\left(j=1,2, \cdots, s^{\prime}\right),
$$

where $s^{\prime}$ is the dimension of the linear system of $(\theta)$ 's of order $n k$ and given characteristic and let $W^{\prime}$ be the singular subvariety corresponding to it. For $k$ great enough, the functions $\theta_{k}\left[h_{/ n}^{/ n}\right](u)$ will not all vanish at the same point. But the functions $\left(\theta_{k}^{i}\left[\left(\begin{array}{l}g+a) / n \\ (h+b) / n\end{array}\right](u)\right)^{n}\right.$ are linear combinations of the $\left(\theta_{n k}^{i}\right)$ 's of our representation. Let $s$ be the dimension of the system of the $\left(\theta_{k}\right)$ 's of given characteristic. We can choose $s$ functions $\left.\theta_{k}\left[\begin{array}{c}(g+a) / n \\ i h+b\end{array}\right) / n\right](u)$ not vanishing at a given point $A$ of $W^{\prime}$. Let us form the matrix which defines $W^{\prime}$ by taking for functions relatively to its $s$ first columns, the $n$th powers of these $s$ functions. One can then verify at once that $A$ must belong to the $W$ which corresponds to the representation by the $\theta_{k}\left[\left(g_{h+a}+a\right) / n\right](u)$. But these subvarieties $W$ are all the transformed of one and the same one by ordinary transformations of the first kind, transformations in as great a number as desired provided $n$ is great enough.* When $n$ exceeds a certain limit, they certainly do not have any common points, hence for the representation then obtained by means of the functions $\theta_{n k}\left[\begin{array}{l}g \\ h\end{array}\right](u)$ with arbitrary characteristic, neither case $(a)$ nor case $(b)$ will present itself, and the theorem is proved.

The importance of this theorem is due to the fact that a number of propositions on varieties of more than two dimensions are not applicable when the singularities are not ordinary. We may mention in particular many of Severi's results as well as the results of our first part. In the sequence, unless otherwise stated, in speaking of an Abelian variety, it will always be under-

* A birational transformation of the first kind of $V_{p}$ is defined by equations such as $u_{i}^{\prime}=u_{i}+$ const. $(i=1,2, \cdots, p)$. 
stood that we mean the variety of rank one without singularities in point to point correspondence without exception with $U_{2 p}$, and it is this variety which we shall designate by $V_{p}$.

\section{§2. Connectivity of varieties of rank one}

52. Let now $u_{1}, u_{2}, \cdots, u_{p}$ be the variables corresponding to $\Omega$ and consider the generalized cube $U_{2 p}$ as defined by the relations

$$
u_{i}=\sum_{\mu=1}^{2 p} t_{\mu} \omega_{i \mu} \quad(i=1,2, \cdots, p),
$$

"here the $(t)$ 's describe the interval $0 \cdots 1$, end points included. $U_{2 p}$ will have for $s$-dimensional elements, the aggregates of points obtained when $2 p-s$ of the $(t)$ 's are equal to zero or one and the others vary. Two elements comf osed of congruent points modulo the periods are homologous. Hence, a s s stem of $\left(\begin{array}{c}2 p \\ p\end{array}\right) s$-dimensional elements not congruent to each other form a ainimum base for all elements of the same dimensionality. Besides, every one if them represents a closed $s$-dimensional manifold, that is, an $s$-cycle, and as these cycles are independent, we shall have a minimum base for s-cycles composed of independent cycles. Hence, for $U_{2 p}$, and therefore for $V_{p}$, $R_{s}=\left(\begin{array}{c}2 p \\ ?\end{array}\right), \sigma_{s}=1$. In particular, $R_{2}=p(2 p-1), \sigma_{1}=\sigma=1$. As a verification for hyperelliptic surfaces, the values $\rho_{0}=5, \rho=1$, were given by Picard, and indeed, $R_{2}=6=\rho_{0}+\rho$.

53. We shall attack the same problem by a slightly different method, which will be found very useful below.

Let us designate by $1,2, \cdots, 2 p$, the edges of $U_{2 p}$, abutting on the origin, and sense each of them in such manner that starting from the origin the direction of advancement be positive. The face $(\mu, \nu)$ will be sensed by sensing its periphery so that the edge $(\mu)$ be a positive segment of it. We then have $(\mu, \nu) \sim-(\nu, \mu)$. From there we can pass to the sensing of a 3-dimensional face $(\mu, \nu, \pi)$, etc. We are after all merely dealing here with Heegaard's "sensed corners".*

To each combination of indices, $i_{1}, i_{2}, \cdots, i_{\text {s }}$ correspond two opposite $s-$ dimensional elements. Let $M_{s}$ be an analytical $s$-cycle. Its projection on $\left(i_{1}, i_{2}, \cdots, i_{s}\right)$ is composed of that element counted several times. I say that if we project it each time on only one of the elements corresponding to the indices $i_{1}, i_{2}, \cdots, i_{s}, M_{8}$ is homologous to the sum of its projections. Let us cut up $M_{.}$, as we may, into a sum of elements homologous to a hypersphere, then project one of these, $M_{s}^{\prime}$, as well as its boundary $M_{s-1}$ upon $\left(i_{1}, i_{2}, \ldots, i_{s}\right)$. This will be done by passing a space $S_{2 p-s}$ through an arbitrary point $A$ of $M_{0}^{\prime}$.

\footnotetext{
*Bulletin de la Société Mathématique de France, vol. 44 (1916), pp. 161-242.
} 
and taking its intersection $B$ with $\left(i_{1}, i_{2}, \cdots, i_{s}\right)$. An arbitrary line (straight or otherwise) joining $A$ to $B$, will be called a projecting line of $A$. It is in no wise necessary that this projecting line be in the $S_{2 p-s}$, but only that in some manner it should be uniquely and continuously defined for all points $A$.

By continuous deformation we may reduce $M^{\prime}$, to its projection $M^{\prime \prime}$, provided we replace it in $M_{*}$ by this projection increased by the $s$-dimensional manifold $M_{\prime \prime}^{\prime \prime}$ generated by the projecting lines of $M_{s-1}$. The manifold $M^{\prime}+M_{0}^{\prime \prime \prime}-M_{\text {, }}^{\prime \prime}$ will form an s-cycle $\bar{M}_{s}$, reducible by deformation to a point, hence the conclusion: If $P$ is an $S_{2 p-s}$ of the $S_{2 p}$ which contains $U_{2 p}$, Poincaré's character belonging to $\bar{M}_{s}$ and $P$ will be $\left(\bar{M}_{s} P\right)=0$. By taking in particular for $P$ a space normal to $\left(i_{1}, i_{2}, \cdots, i_{s}\right)$ it is seen that the projection of $\bar{M}_{s}$ on $\left(i_{1}, i_{2}, \cdots, i_{s}\right)$ is composed of mutually opposite elements. Hence, finally, when $M_{0}^{\prime}$, is replaced in $M_{s}$ by $M_{0}^{\prime \prime}-M_{0}^{\prime \prime \prime}$, the projections on the $s$-dimensional elements of $U_{2 p}$ are not changed. Proceeding similarly with all the $s$-dimensional elements such as $M^{\prime}$, of which $M_{s}$ is composed, we shall succeed in replacing finally this cycle by $\left(i_{1}, i_{2}, \cdots, i_{s}\right)$ counted a certain number of times and an $s$-cycle whose projection on $\left(i_{1}, i_{2}, \cdots, i_{s}\right)$ has less than $s$ dimensions while on any other $s$-dimensional element, the projection is the same as for $M_{s}$. We may reason similarly with the part of this new cycle exterior to $\left(i_{1}, i_{2}, \cdots, i_{s}\right)$ relatively to an element upon which it has an $s$-dimensional projection and so on, so that finally $M_{s}$ will have been replaced by the sum of its projections. The theorem is therefore proved.

Remark. The proof holds also when we consider the projections on elements derived from those such as $\left(i_{1}, i_{2}, \cdots, i_{s}\right)$ by translation.

The $s$-cycles are equal to sums of multiples of the cycles $\left(i_{1}, i_{2}, \cdots, i_{s}\right)$ which therefore form a minimum base. To establish the independence of the cycles of this base, it is sufficient to remark with Picard, that the integral $\mathcal{S} \mathcal{S} \cdots \mathcal{S} d t_{i_{1}} d t_{i_{3}} \cdots d t_{i_{\text {g }}}$ has a period +1 relatively to $\left(i_{1}, i_{2}, \cdots, i_{s}\right)$ and 0 relatively to any other cycle. We have thus a system of $\left({ }^{2} p\right)$ integrals with a period matrix relatively to the cycles in question of rank equal to the number of these periods. As these integrals have no periods relatively to the cycles homologous to zero, the $\left(\begin{array}{c}{ }^{2} p \\ p\end{array}\right)$ cycles thus obtained are independent and form a minimum base, hence again $R_{s}=\left(\begin{array}{c}2 p \\ s\end{array}\right), \sigma_{s}=1$.

54. The s-uple integrals of the first kind of $V_{p}$, as is well known for $p=2$ and easily proved for any $p$, are all linear combinations of the integrals

$$
\iint \cdots \int d u_{j_{1}} d u_{j_{2}} \cdots d u_{j_{\bullet}}
$$

Their number is therefore $i_{z}=\left(\begin{array}{l}p \\ q\end{array}\right)$. To say that there is a certain number of $s$-cycles with periods of integrals of the first kind all zero is therefore the same as to say that there are as many linear relations with integral coefficients 
between the determinants of order $s$ derived from the array

$\begin{array}{llll}\omega_{j_{1}, 1} & \omega_{j_{1}, 2} & \cdots & \omega_{j_{1}, 2 p} \\ \omega_{j_{2}, 1} & \cdot & \cdots & \cdot \\ \cdot & \cdot & \cdot & \cdot \\ \omega_{j_{2}, 1} & \cdot & \cdots & \omega_{j_{\ell}, 2 p},\end{array}$

the coefficients being independent of the indices $j_{1}, j_{2}, \cdots, j_{2 p}$.

In the case $s=2$, the number of these independent relations is precisely the number $1+k$ of alternate Riemann forms belonging to $\Omega$. We have therefore for $V_{p}$ according to Part I, $\rho \leqq 1+k$. The consideration of intermediary functions will allow us to show that $\rho \geqq 1+k$. Assuming this result, for the present, we have then $\rho=1+k$. In other words, every two-cycle of $V_{p}$ without periods of integrals of the first kind, is algebraic. For $p=2$, this has already been established by Bagnera and de Franchis. Thus, the theory of Part I leads us naturally to the consideration of the alternate Riemann forms of $\Omega$.

55. Let us reduce $\Omega$ to the canonical matrix $A$. In general, there will be no other relations between the periods of the unique $p$-uple integral of the first kind of $V_{p}$ than the following: Let

$$
\left|a_{i_{\alpha}, j_{\beta}}\right|, \quad\left|a_{j_{\beta}, i_{a}}\right| \quad(\alpha, \beta=1,2, \cdots, s)
$$

be two minors of the determinant $\left|a_{j h}\right|(j, h=1,2, \cdots, p)$, symmetrical relatively to the principal diagonal, then denote for the present by $\omega_{i \mu}$ the term of the matrix $A$ at the intersection of the $i$ th row and $\mu$ th column. To the first of our minors corresponds the following determinant of order $p$ derived from $A$ :

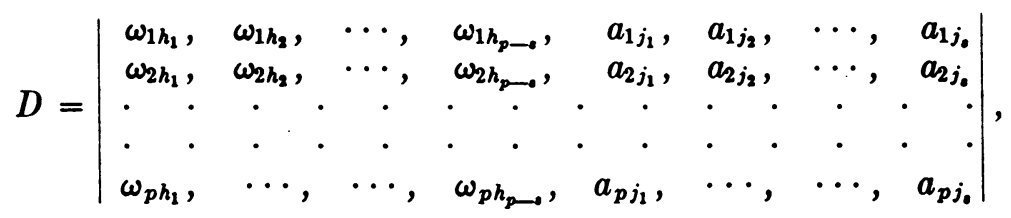

where the $(h)$ 's are integers in increasing order such that $h_{1}, h_{2}, \cdots, h_{p-s}$, $j_{1}, j_{2}, \cdots, j_{s}$, form in some order the sequence, $1,2, \cdots, p$. Similarly there corresponds to the other minor a determinant of order $p, D^{\prime}$, derived from $A$ by adjunction of certain columns of indices $k_{1}, k_{2}, \cdots, k_{p-s}$, at most equal to $p$. One verifies at once that $e_{h_{1}} \cdot e_{h_{2}} \cdots e_{h_{p-s}} \cdot D=e_{k_{1}} \cdot e_{\bar{k}_{2}} \cdots e_{k_{p-s}} \cdot D^{\prime}$. When $A$ is as general as possible, every linear relation with integral coefficients existing between the determinants of order $p$ derived from $A$ reduces to a combination of those of that type which result immediately from the fact that $\left|a_{j h}\right|(j, h=1,2, \cdots, p)$ is a symmetrical determinant. Their number is equal to half the number of non-symmetrical minors derived from the pre- 
ceding determinant, that is to

$$
\frac{1}{2} \sum_{j=1}^{p}\left(\begin{array}{c}
p \\
j
\end{array}\right)\left[\left(\begin{array}{c}
p \\
j
\end{array}\right)-1\right]=\frac{1}{2}\left(\begin{array}{c}
2 p \\
p
\end{array}\right)-2^{p-1} .
$$

This is the number of $p$-cycles with zero periods for the $p$-uple integral of the first kind of $V_{p}$ when the variety is as general as possible.

Let us start from the relation

$$
(1+i)^{2 p}=2^{p} i^{p}=\sum_{j=0}^{p}\left(\begin{array}{c}
2 p \\
j
\end{array}\right) i^{j}
$$

By considering separately the two cases where $p$ is even or odd, we find that

$$
\frac{1}{2}\left(\begin{array}{c}
2 p \\
p
\end{array}\right)-2^{p-1}=R_{p-2}-R_{p-4}+R_{p-6}-\cdots,
$$

and therefore according to Part I, $\rho_{p}=0$.

A similar though somewhat more complicated discussion which we have not carried out in detail leads no doubt to $\rho_{s}=0$. Thus the numbers $\rho_{\mathrm{s}}$ of the most general Abelian variety of rank one are all zero.

For a special $V_{p}$ the number of $s$-cycles with zero periods for the integrals of the first kind may exceed the above. Let generally,

$$
k_{s}+R_{s-2}-R_{s-4}+\cdots
$$

be this number, $k_{s} \equiv 0$. The integer $k_{s}$ may be called the $s$-dimensional index of singularity of $V_{p}$ or $\Omega$. We always have

$$
\rho_{s} \leqq k_{s}, \quad \rho_{s}^{0} \equiv\left(\begin{array}{c}
2 p \\
s
\end{array}\right)-\left(\begin{array}{c}
2 p \\
s-2
\end{array}\right)-k_{s} .
$$

We may of course introduce an s-dimensional index of multiplication. All these indices would no doubt be useful for the classification of Abelian varieties and many of the propositions given by Scorza for $s=2$ could be extended to them but we shall not discuss this any further.

\section{§ 3. Intermediary functions}

57. An entire function $\phi\left(u_{1}, u_{2}, \cdots, u_{p}\right)$ belonging to a matrix $\Omega$ with $p$ rows and $2 p$ columns is said to be an intermediary function if

$$
\phi\left(u+\omega_{\mu}\right)=e^{-2 \pi i\left(\Sigma_{j=1}^{p} a_{j \mu} n_{j}+\beta_{\mu}\right)} \cdot \phi(u) \quad(\mu=1,2, \cdots, 2 p) .
$$

By comparing the two possible values for $\phi\left(u+\omega_{\mu}+\omega_{\nu}\right)$ the following equations of condition are obtained:

$$
\sum_{j=1}^{p}\left(\alpha_{j_{\mu}} \omega_{j_{\nu}}-\alpha_{j_{\nu}} \omega_{j_{\mu}}\right)=m_{\mu \nu} \quad(\mu, \nu=1,2, \cdots, 2 p) .
$$


where the $(m)$ 's are integers. Since $m_{\mu \nu}=-m_{\nu \mu}$ the form

$$
F=\sum_{\mu, \nu=1}^{2 p} m_{\mu \nu} x_{\mu} y_{\nu}
$$

is an alternate form with integral coefficients. We shall call it the fundamental form belonging to $\phi$. It will play a very important part in the sequence.

58. The second derivatives of $\log \phi$ are meromorphic periodic functions belonging to $\Omega$. We shall assume that they are effectively $2 p$-ply periodic, which is permissible, for otherwise we could replace $\Omega$ by the matrix of genus $<p$, formed by the primitive periods. $\Omega$ will then be a Riemann matrix of genus $p$.

If $\phi$ is a function which vanishes nowhere, the second derivatives of $\log \phi$ are entire periodic functions and therefore they are constants, hence $\phi$ is of the form $e^{G(u)}$, where $G$ is a quadratic polynomial in the $(u)$ 's. Let us assume that $\phi$ is not of this type. The second derivatives of $\log \phi$ will then be of type $\theta_{m} / \theta_{m}^{\prime}$ and there will be a hypersurface $E$ of $V_{p}$ determined by $\phi=0$, or, as we shall say, cut out by $\phi$. By a $\theta_{m}$ we mean a $\theta$-function belonging to a canonical matrix $A$ equivalent to $\Omega$. In terms of the $(u)$ 's it is a function

$$
\theta_{m}\left(\lambda_{1} u_{1}+\lambda_{2} u_{2}+\cdots+\lambda_{p} u_{p}\right)
$$

in reality an intermediary function derived from a $\theta$.

The hypersurface cut out by $\theta_{m}^{\prime}$ is composed of $E$ and of another hypersurface $E^{\prime}$ upon which $\theta_{m}$ vanishes also. As $\theta_{m}, \theta_{m}^{\prime}$, cut out algebraic hypersurfaces, their common part $E^{\prime}$ is also algebraic. Hence, $E$, the residue of an algebraic hypersurface with respect to another, is itself algebraic and therefore any intermediary function cuts out in $V_{p}$ an algebraic hypersurface.

59. For the sequence, it is very important to show that an arbitrary intermediary function is always reducible to a $\dot{\theta}$ by a linear change of variables. This reduction has been very simply effected for $p=2$ by Humbert, Bagnera and de Franchis. Humbert's method may be extended to any $p$, but as it consists in taking $\Omega$ always in the canonical form, we do not obtain thus in any simple manner the relationship between fundamental and principal forms. As to the method of Bagnera and de Franchis, we have not succeeded in extending it. The difficulty consists in showing that a certain form is principal. The method to be followed here, completely different from that of these authors, seems to present considerable interest in itself and probably will be useful in other applications as well.

This method consists in the following: We shall obtain the expression of the algebraic cycle $\left(E^{p-1}\right)$ in terms of the cycles $(\mu, \nu)$, and from the expression in question, we shall deduce that the inverse of the fundamental form is a principal form of Riemann. 
60. In the first place, the hypersurface $(E)$ itself is a $(2 p-2)$-cycle. We shall first seek the expression of this cycle $(E)$ in terms of the $p(2 p-1)$ fundamental cycles. Let $(E) \sim \Sigma m_{i_{1} i_{2} \cdots i_{2 p-2}}\left(i_{1}, i_{2}, \cdots, i_{2 p-2}\right)$. In the sum at the right, only one of the cycles corresponding to a given combination of indices must be taken. We shall agree to take always the cycle for which the ( $i$ )'s are in increasing order. The question is to evaluate the coefficients in the homology. We shall see that if $E$ is irreducible, a hypothesis which will be made until further notice, $(E)$ considered as a manifold entirely in $U_{2 p}$ is sensed everywhere in the same manner with respect to the $(2 p-2)$ dimensional elements of $U_{2 p}$, which translates itself into the fact that its projections on $\left(i_{1}, i_{2}, \cdots, i_{2 p}\right)$ do not include any $(2 p-2)$ dimensional elements which destroy each other. It will be necessary then, first to determine that sense, then the number of times that the projection on $\left(i_{1}, i_{2}, \cdots, i_{2 p-2}\right)$ covers it up.

61. We must first examine a little closer the notion of sense. Let there be given in an $S_{q}$ a system of $q$ directed rectangular axes and corresponding variables $t_{1}, t_{2}, \cdots, t_{q}$ and in that space an analytical manifold of $k<q$ dimensions, $M_{k}$, without any singular points. We wish to define what is meant by the sensing of that element with respect to the $S_{k}$ defined by the equations $t_{i_{1}}=t_{i_{2}}=\cdots=t_{i_{q-k}}=0$, which we will designate by $\left(i_{q-k+1}, i_{q-k+2}, \cdots, i_{q}\right)$, it being understood that $t_{i_{q-k+1}}, \cdots, t_{i_{q}}$ are variable in that space.

Let $M_{q-k}^{\prime}$ be an $S_{q-k}$ transverse to $M_{k}$ and passing through one of its points $A$ and take a system of $q$ axes, of which $q-k, A s_{1}, A s_{2}, \cdots, A s_{q-k}$ are in $M_{q-k}^{\prime}$ and $k$ others $A s_{q-k+1}, A s_{q-k+2}, \cdots, A s_{q}$ are in $M_{k}$. More precisely the coordinates of any point of $M_{k}$ are, in the vicinity of $A$, analytical functions of certain variables $s_{q-k+i}(i=1,2, \cdots, k)$. We may define the "axes in $M_{k}$ " as the tangents at $A$ to the lines $d s_{q-k+i} \neq 0, d s_{q-k+j}=0$ $(j \neq i)$, sensed positively in the direction of increreasing $(s)$ 's.-Assume first that the axes $t_{i_{h}}$ are congruent to those just defined, i.e. if $t_{h}^{\prime}$ are the variables for this second set, that there exists a continuous series of infinitesimal transformations of the group with parameters $a_{i k}, b_{i}$,

$$
t_{i}^{\prime}=\sum_{k=1}^{q} a_{i k} t_{i_{k}}+b_{i}, \quad\left|a_{i k}\right|=+1 \quad(i, k=1,2, \cdots, q)
$$

reducing the first axes to the second. This continuous series of transformations defines a displacement in $S_{q}$. The Jacobian is

$$
\frac{D\left(s_{1}, s_{2}, \cdots, s_{q}\right)}{D\left(t_{i_{1}}, t_{i_{2}}, \cdots, t_{i_{q}}\right)}=+1
$$

If there exists such a displacement for which the Jacobian

$$
\frac{D\left(t_{1}^{\prime}, t_{2}^{\prime}, \cdots, t_{q-k}^{\prime}\right)}{D\left(t_{i_{1}}, t_{i_{0}}, \cdots, t_{i_{q-k}}\right)}
$$


changes its sign an even number of times, we shall say that $M_{k}$ is sensed positively with respect to the initial space, $\left(i_{q-k+1}, \cdots, i_{q}\right)$. In such a displacement, a sufficiently small $k$-dimensional element within the displaced space $\left(i_{q-k+1}, \cdots, i_{q}\right)$ is never projected within its initial position into an element of $<k$ dimensions or is so an even number of times. Finally, the product

$$
\frac{D\left(s_{1}, s_{2}, \cdots, s_{q-k}\right)}{D\left(t_{i_{1}}, t_{i_{2}}, \cdots, t_{i_{q-k}}\right)} \cdot \frac{D\left(s_{1}, s_{2}, \cdots, s_{q}\right)}{D\left(t_{i_{1}}, t_{i_{1}}, \cdots, t_{i_{q}}\right)}>0 .
$$

If on the contrary the sign considered above changes an odd number of timesand it is easy to show that its parity is perfectly defined-we shall say that the sense in question is negative. The product of the two Jacobians is then negative.

If the axes $(s)$ and $(t)$ are not congruent, we compare $M_{k}$ to the $S_{k}$ corresponding to the indices $i_{2}, i_{1}, i_{3}, \cdots, i_{q}$ which is opposed to $\left(i_{q-k+1}, \cdots, i_{q}\right)$ and we define the sense as being opposite to that then obtained. We see then that the sense is always given by the sign of the Jacobian product, which may therefore be used as definition of this sense. The axes $(s)$ will be called intrinsic axes of $M_{k}$.

62. Let us return to our problem. To show first that $(E)$ has an invariant sense relatively to $\left(i_{3}, i_{4}, \cdots, i_{2 p}\right)$ it is sufficient to establish that if $s_{1}, s_{2}$, $\cdots, s_{2 p}$ is a system of intrinsic axes and $s_{1}, s_{2}$, the variables belonging to the transverse plane the Jacobians $D\left(s_{1}, s_{2}\right) / D\left(t_{i_{1}}, t_{i_{1}}\right)$ vanish only on a manifold of $(2 p-4)$ dimensions at most, of $(E)$. Let

$$
\phi=\phi^{\prime}+i \phi^{\prime \prime}, \quad u_{j}=u_{j}^{\prime}+i u_{j}^{\prime \prime},
$$

where $\phi^{\prime}, \phi^{\prime \prime}, u_{j}^{\prime}, u_{j}^{\prime \prime}$ are real, and take as coördinates $s_{1}, s_{2}$, two distinct linear combinations of $\phi^{\prime}, \phi^{\prime \prime}$. The question is reduced to showing then that the Jacobian $D\left(\phi^{\prime}, \phi^{\prime \prime}\right) / D\left(t_{1}, t_{2}\right)$, for example, vanishes only on a $(2 p-4)$ dimensional manifold of $(E)$. But the left hand side is a linear combination with constant coefficients of products of terms such as $\partial \phi^{\prime} / \partial u_{j}^{\prime}, \partial \phi^{\prime} / \partial u_{j}^{\prime \prime}$, $\partial \phi^{\prime \prime} / \partial u_{j}^{\prime}, \partial \phi^{\prime \prime} / \partial u_{j}^{\prime \prime}$, that is, by virtue of classical relations, of terms $\partial \phi / \partial u_{j}$. It is therefore an entire function of $u_{1}, u_{2}, \cdots, u_{p}$ and the dimension of the manifold in question is at most $2 p-4$.

It follows from this that $m_{i_{3} i_{6} \cdots i_{z_{3}}}$ has the sign of the product

$$
\frac{D\left(t_{i_{1}}, t_{i_{2}}\right)}{D\left(s_{1}, s_{2}\right)} \cdot \frac{D\left(t_{i_{1}}, t_{i_{2}}, \cdots, t_{i_{p}}\right)}{D\left(s_{1}, s_{2}, \cdots, s_{2 p}\right)} \text {. }
$$

But the second factor is equal to $(-1)^{n} D\left(t_{1}, t_{2}, \cdots, t_{2 p}\right) / D\left(s_{1}, s_{2}, \cdots, s_{2 p}\right)$

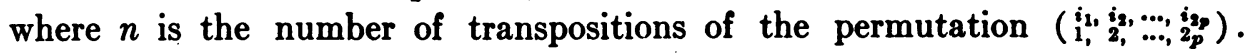
Finally, we can always choose the $(s)$ 's in such a way that the coefficient of 
$(-1)^{n}$ will be positive. In these conditions $m_{i_{g} i_{q} \cdots i_{2 p}}$ will have the sign of $(-1)^{n} D\left(t_{i_{1}}, t_{i_{2}}\right) / D\left(s_{1}, s_{2}\right)$.

To determine the absolute value of $m_{i_{3} i_{4} \cdots i_{z_{p}}}$, we remark that the number of times that the projection of an $(E)$ on an $\left(i_{3}, i_{4}, \cdots, i_{2 p}\right)$ covers it up, is equal to the a'ssolute value of

$$
\frac{1}{2 \pi i} \int d \log \phi
$$

taken over the positive contour of $\left(i_{1}, i_{2}\right)$. The value as found by an elementary integration is* $\sum_{j=1}^{p}\left(\alpha_{j i_{1}} \omega_{j i_{2}}-\alpha_{j i_{2}} \omega_{j i_{1}}\right)=m_{i_{1} i_{2}}$. Now let us take in the transverse plane, a circuit $\zeta$ surrounding its point of incidence and such that

$$
\frac{1}{2 \pi i} \int_{\zeta} d \log \phi=+1 .
$$

We shall assume the axes $s_{1}, s_{2}$, so chosen that $\zeta$ is sensed positively in the direction $s_{1}, s_{2}$.

Let $\zeta^{\prime}$ be the projection of $\zeta$ on $\left(i_{1}, i_{2}\right)$ when the point of incidence of the transverse plane comes in one of the points where $(E)$ cuts $\left(i_{1}, i_{2}\right)$. We have

$$
\frac{1}{2 \pi i} \int_{\zeta} d \log \phi=\frac{1}{2 \pi i} \int_{\zeta_{1}} d \log \phi=+1 .
$$

Hence the integral taken over the contour of $\left(i_{1}, i_{2}\right)$ in the sense from $i_{1}$ towards $i_{2}$, is positive if $\zeta^{\prime}$ and the contour have the same sense, negative in the contrary case. Therefore

$$
m_{i_{1} i_{2}} \frac{D\left(t_{i_{1}}, t_{i_{2}}\right)}{D\left(s_{1}, s_{2}\right)}>0,
$$

and finally $m_{i_{3} i_{1} \cdots i_{z_{p}}}=(-1)^{n} m_{i_{1} i_{2}}$. If $n^{\prime}$ corresponds to the indices $i_{2}, i_{1}, \cdots, i_{2 p}$ we have $(-1)^{n}=-(-1)^{n^{\prime}},(-1)^{n} m_{i_{1} i_{2}}=(-1)^{n^{\prime}} m_{i_{2} i_{1}}$, and we can decide without inconvenience to take $i_{1}<i_{2}$ since we must choose only one of these combinations. In assuming, as we already had occasion to state, $i_{3}<i_{4}<\cdots<i_{2 p}, n$ will be the number of transpositions which bring $i_{1}, i_{2}$, into the position 1,2 . Finally then

$$
(E) \sim \sum(-1)^{n} m_{i_{1} i_{3}}\left(i_{3}, i_{4}, \cdots, i_{2 p}\right) .
$$

63. Let us show that with a suitable convention nothing is changed, even if $E$ is reducible.

I say first, that two arbitrary hypersurfaces, $\left(E^{\prime}\right),\left(E^{\prime \prime}\right)$ have two sides sensed alike with respect to all the $\left(i_{3}, i_{4}, \cdots, i_{2 p}\right)$. For, taking always a

* Poincaré, as I have found since writing the above, has already used analagous considerations in a memoir of the A cta mathe matica, vol. 26 (1902), pp. 43-98. 
representation analogous to (5) of No. 50 for $V_{p}$, there exists a polynomial $F\left(x_{1}, x_{2}, \cdots, x_{8}\right)$ vanishing on $E^{\prime}$ and $E^{\prime \prime}$. Replacing the $(x)$ 's by the $(\theta)$ 's, we see that $E^{\prime}, E^{\prime \prime}$, are part of an irreducible hypersurface cut out by a certain $\theta$. Hence $\left(E^{\prime}\right),\left(E^{\prime \prime}\right)$, have an invariant sense at all their points. Moreover, there exists an algebraic variety of the $S_{s-1}$, containing $V_{p}$, cutting out on $V_{p}$ an irreducible hypersurface, $G$, tangent to $E^{\prime}, E^{\prime \prime}$ in two ordinary points, $A, B$. If we prefer, we may say that there is a suitable $\theta$ cutting cut $G$. Let us now take on $\left(E^{\prime}\right),\left(E^{\prime \prime}\right),(G)$, intrinsic coördinates with the corresponding axes congruent to each other, and let $s_{j}^{\prime}, s_{j}^{\prime \prime}, s_{j}$ be these coördinates. The senses with respect to $\left(i_{3}, i_{4}, \cdots, i_{2 p}\right)$ of $\left(E^{\prime}\right),(G)$ in $A$ and of $\left(E^{\prime \prime}\right),(G)$ in $B$ will be the same or opposite according as the Jacobians $D\left(s_{1}^{\prime}, s_{2}^{\prime}\right) / D\left(s_{1}, s_{2}\right), D\left(s_{1}^{\prime \prime}, s_{2}^{\prime \prime}\right) / D\left(s_{1}, s_{2}\right)$ are positive or negative, the first in $A$ and the second in $B$. As these signs do not depend upon the (i)'s, our affirmation follows.

The preceding discussion makes it possible to define a typical sensing for algebraic hypersurfaces of $V_{p}$ to be taken as positive, and this we shall do in the future.

Let us assume that $\left(E^{\prime}\right),\left(E^{\prime \prime}\right)$, are two irreducible parts of the hypersurface $(E)$ cut out by $\phi$ and that they intersect in a point, $A$, ordinary for both. Through $A$ we draw a plane parallel to $\left(i_{1}, i_{2}\right)$ wherein we trace a small circuit $\zeta$ surrounding $A$. By attributing to $\zeta^{\prime}$ a suitable direction, we shall have $\int_{5} d \log \phi=+2 \cdot 2 \pi i$. Through a point near $A$, let us draw a plane transverse to $\left(E^{\prime}\right)$, then surround its point of incidence with a small circuit $\zeta^{\prime}$, avoiding $\left(E^{\prime \prime}\right)$ and sensed in such a way that

$$
\int_{\zeta^{\prime}} d \log \phi=2 \pi i \text {. }
$$

Let $\zeta^{\prime \prime}$ be an analogous circuit for $\left(E^{\prime \prime}\right)$. As $\mathcal{S}_{\zeta} d \log \phi=\mathcal{S}_{\zeta}^{\prime}+\mathcal{S}_{\zeta}^{\prime \prime}$, $\zeta^{\prime}, \zeta^{\prime \prime}$ are sensed similarly with respect to all the $\left(i_{1}, i_{2}\right)$. If, therefore, we desire once for all to take for every hypersurface only the cycle formed by its positive side, the Jacobians $D\left(t_{i_{1}}, t_{i_{2}}\right) / D\left(s_{1}, s_{2}\right)$ will still have the same sign at all points of $(E)$ provided the intrinsic axes are congruent at all points. These signs will always be those of the coefficients $m_{i_{1} i_{2}}$, and our reasoning still holds, leading again to the same homology as previously.

Let us assume, finally, that $\left(E^{\prime}\right),\left(E^{\prime \prime}\right)$, have no simple points in common, and let $\psi$ be an intermediary function cutting out a hypersurface $G$, which meets $E^{\prime}, E^{\prime \prime}$ in two ordinary points, $A^{\prime}, A^{\prime \prime}$. By reasoning as we have just done, with $\phi \psi$ taking now the place of $\phi$, and comparing $E^{\prime}$ and $E^{\prime \prime}$ with $G$, we show at once that we can always arrange matters so that the Jacobians $D\left(t_{i_{1}}, t_{i_{2}}\right) / D\left(s_{1}, s_{2}\right)$ have the same sign on $\left(E^{\prime}\right)$ and $\left(E^{\prime \prime}\right)$, the corresponding intrinsic axes being always assumed congruent. The rest can be completed as previously. 
Remark. An immediate consequence of the similar sensing of the hypersurfaces of $V_{p}$ is that the signs of the coefficients $m_{i_{1} i_{z}}$ depend only upon the indices but not upon the fundamental form considered. The sign of $(-1)^{n} m_{i_{1} i_{2}}$ indicates the sensing of the algebraic hypersurfaces of $V_{p}$ relatively to $\left(i_{3}, i_{4}, \cdots, i_{2 p}\right)$.

64. It is clear that, in the same manner as above for a $2 p-2$ dimensional element, the sensing of a $2 p-4$ dimensional element with respect to $\left(i_{6}, i_{6}, \cdots, i_{2 p}\right)$ can be considered as determined by the sign of the product

$$
\frac{D\left(t_{i_{1}}, t_{i_{2}}, t_{i_{3}}, t_{i_{4}}\right)}{D\left(s_{1}, s_{2}, s_{3}, s_{4}\right)} \cdot \frac{D\left(t_{i_{1}}, t_{i_{2}}, \cdots, t_{i_{p}}\right),}{D\left(s_{1}, s_{2}, \cdots, s_{2 p}\right)}
$$

where the coördinates $s_{1}, s_{2}, s_{3}, s_{4}$ belong to the transverse $S_{4}$ and the others belong to the element itself.

We can show next that the image in $U_{2 p}$ of an irreducible $p-2$ dimensional algebraic subvariety of $V_{p}$ is a $2 p-4$ dimensional manifold sensed relatively to the $\left(i_{5}, i_{6}, \cdots, i_{2 p}\right)$, in a manner invariant in all its points and independent of the subvariety chosen.

Let us consider finally several such subvarieties belonging to the intersection of two algebraic hypersurfaces $E_{1}, E_{2}$. At all their points we can take intrinsic coördinates, $s_{1}, s_{2}, \cdots, s_{2 p}$, such that $s_{1}, s_{2}$ belong to the plane transverse to $\left(E_{1}\right), s_{3}, s_{4}$ to the plane transverse to $\left(E_{2}\right)$, and such that, moreover, the Jacobians

$$
\frac{D\left(t_{1}, t_{2}, \cdots, t_{2 p}\right)}{D\left(s_{1}, s_{2}, \cdots, s_{2 p}\right)}, \frac{D\left(t_{i_{1}}, t_{i_{2}}\right)}{D\left(s_{1}, s_{2}\right)}, \frac{D\left(t_{i_{1}}, t_{i_{z}}\right)}{D\left(s_{3}, s_{4}\right)}
$$

should be, the first equal to one, and the other two of invariant sign at all points of the intersection of $\left(E_{1}\right)$ with $\left(E_{2}\right)$. This is equivalent to associating in a definite way, $\left(E_{1}\right),\left(E_{2}\right)$, in their common points. The known identity

$$
\frac{D\left(t_{i_{1}}, t_{i_{2}}, t_{i_{3}}, t_{i_{4}}\right)}{D\left(s_{1}, s_{2}, s_{3}, s_{4}\right)}=\sum \pm \frac{D\left(t_{i_{1}}, t_{i_{3}}\right)}{D\left(s_{a}, s_{\beta}\right)} \cdot \frac{D\left(t_{i_{3}}, t_{i_{4}}\right)}{D\left(s_{\gamma}, s_{\delta}\right)}
$$

shows that we change the sense of the intersection whenever we change the sense of one of the hypersurfaces but not when we change that of both. We shall agree always to associate the sides of $\left(E_{1}\right),\left(E_{2}\right)$ which have the same sense. The side thus obtained for their intersection will be said to be its positive side. The sum of the irreducible parts similarly sensed will be what we shall call the intersection $\left(E_{1} E_{2}\right)$. The following are two of its properties: $(a)$ The cycles $\left(E_{1} E_{2}\right)$ and $\left(E_{2} E_{1}\right)$ are identical; (b) let there be two cycles $\Delta_{1}, \Delta_{2}$, nowhere sensed in opposition to $\left(E_{1}\right),\left(E_{2}\right)$, and respectively reducible to these two cycles by a deformation, during which they never acquire a common $2 p-3$ dimensional part. We may define their common $(2 p-4)$-cycle $\Delta_{1} \Delta_{2}$ as we have done for $\left(E_{1}\right),\left(E_{2}\right)$, and this cycle is homologous to $\left(E_{1} E_{2}\right)$. 
65. Let us designate for the present by $\left(i_{1}, i_{2}, \cdots, i_{s}\right)$ not merely an element forming part of the boundary of $U_{2 p}$ but more generally any element which may be derived from it by a translation parallel to an edge-such an element forming of course a homologous cycle.

The hypersurfaces $E_{1}, E_{2}$, are cut out by intermediary functions belonging to two fundamental forms

$$
F_{1}=\sum m_{\mu \nu}^{1} x_{\mu} y_{\nu}, \quad F_{2}=\sum m_{\mu \nu}^{2} x_{\mu} y_{\nu} .
$$

We can take for cycles $\Delta_{1}, \Delta_{2}$, the following:

$\Delta_{1}=\sum(-1)^{n} m_{i_{1} i_{2}}^{1}\left(i_{3}, i_{4}, \cdots, i_{2 p}\right), \Delta_{2}=\sum(-1)^{n} m_{i_{1} i_{2}}^{2}\left(i_{z}^{\prime}, i_{4}, \cdots, i_{3 p}^{\prime}\right)$,

where at the right the cycles are so chosen that $\Delta_{1}, \Delta_{2}$ do not have any $(2 p-3)$-dimensional manifold in common.

Owing to the large degree of arbitrariness in the definition of the projecting lines in No. 53, we may first reduce $\left(E_{1}\right)$ to $\Delta_{1}$, then $\left(E_{2}\right)$ to $\Delta_{2}$ in such a manner that during the deformation the two cycles never acquire a common $(2 p-3)$-dimensional manifold. The property $(b)$ is then applicable here and we shall have $\left(\Delta_{1} \Delta_{2}\right) \sim\left(E_{1} E_{2}\right)$.

The question is therefore reduced to the determination of the $(2 p-4)$ dimensional cycles common to the $\left(i_{3}, i_{4}, \cdots, i_{2 p}\right)$. Now consider the elements $\left(i_{3}, i_{4}, \cdots, i_{2 p}\right),\left(i_{3}^{\prime}, i_{4}^{\prime}, \cdots, i_{2 p}^{\prime}\right)$. They are respectively represented by two systems of equations

$$
x_{i_{1}}=\text { const., } \quad x_{i_{2}}=\text { const.; } \quad x_{i_{1}^{\prime}}^{\prime}=\text { const., } \quad x_{i_{2}^{\prime}}^{\prime}=\text { const., }
$$

where the two sequences $i_{1}, i_{2}, \cdots, i_{2 p}$ and $i_{1}^{\prime}, i_{2}^{\prime}, \cdots, i_{2 p}^{\prime}$ form each in a certain order the sequence of integers, $1,2, \cdots, 2 p$. These equations will be compatible if and only if the four indices, $i_{1}, i_{2}, i_{1}^{\prime}, i_{2}^{\prime}$ are all different. We will then have intersection of the cycles $\left(i_{3}, i_{4}, \cdots, i_{2 p}\right),\left(i_{3}^{\prime}, i_{4}^{\prime}, \cdots, i_{2 p}^{\prime}\right)$ only if all their indices except two are the same. Let $i_{a}, i_{\beta}$ be those of the first which do not belong to the second, $i_{\gamma}^{\prime}, i_{\delta}^{\prime}$ those of the second which do not belong to the first, and $n, n^{\prime}$ the numbers of transpositions bringing these pairs of indices into the position 1,2 , from their right place in the sequence $1,2, \cdots, 2 p$. We can replace $\left(E_{1}\right),\left(E_{2}\right)$, by the sums of homologous cycles. In the one belonging to $\left(E_{1}\right)$ we have the term

$$
(-1)^{n^{\prime}} m_{i_{\gamma}^{\prime} i_{\delta}^{\prime}}^{\prime}\left(i_{3}, i_{4}, \cdots, i_{2 p}\right)
$$

and in that belonging to $\left(E_{2}\right)$, the term $(-1)^{n} m_{i_{\alpha} i_{\beta}}\left(i_{3}^{\prime}, i_{4}^{\prime}, \cdots, i_{2 p}^{\prime}\right)$, and in no other terms of the sum do the same cycles appear. Let $j_{5}, j_{6}, \cdots, j_{2 p}$ be the common indices. These two terms can be replaced by

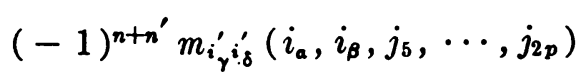


and $(-1)^{n+n^{\prime}} m_{i_{a} i_{\beta}}\left(i_{\gamma}^{\prime}, i_{\delta}^{\prime}, j_{5}, \cdots, j_{2 p}\right)$ respectively. Their intersection is composed of the cycle

$$
m_{i_{a} i_{\beta}}^{2} m_{i_{\gamma}^{\prime} i_{\delta}^{\prime}}^{1} \frac{D\left(t_{i_{\alpha}}, t_{i_{\beta}}, t_{i_{\gamma}^{\prime}}, t_{i_{\delta}^{\prime}}^{\prime}, t_{j_{b}}, \cdots, t_{j_{z_{p}}}\right)}{D\left(t_{1}, t_{2}, \cdots, t_{2 p}\right)}\left(j_{b}, j_{b}, \cdots, j_{2 p}\right),
$$

the Jacobian being introduced as above for reasons of sensing. This term is of the type

$$
(-1)^{n} m_{i_{1} i_{2}}^{1} m_{i_{3} i_{4}}^{2}\left(i_{5}, i_{6}, \cdots, i_{2 p}\right) \text {, }
$$

where $n$ is always the same number and can be taken to be equal to the sum of the transpositions bringing the pairs $i_{1}, i_{2}$ and $i_{3}, i_{4}$ each from their proper places in the sequence $1,2, \cdots, 2 p$ into the position 1,2 . If we assume each of the three systems of indices $i_{1}, i_{2} ; i_{3}, i_{4} ; i_{5}, i_{6}, \cdots, i_{2 p}$, in increasing order, we shall have

$$
\left(E_{1} E_{2}\right) \sim \sum(-1)^{n} m_{i_{1} i_{2}}^{1} m_{i_{3} i_{4}}^{2}\left(i_{6}, i_{6}, \cdots, i_{2 p}\right)
$$

and in particular if we decide always to take $i_{1}<i_{3}$

$$
\left(E^{2}\right) \sim 2 \sum(-1)^{n} m_{i_{1} i_{2}} m_{i_{3} i_{4}}\left(i_{5}, i_{6}, \cdots, i_{2 p}\right) .
$$

This can be generalized at once and we shall have with conventions similar to those already made

$$
\begin{aligned}
\left(E_{1} E_{2} \cdots E_{s}\right) & \sim \sum(-1)^{n} m_{i_{1} i_{8}}^{1} m_{i_{s} i_{4}}^{2} \cdots m_{i_{2 s-1} i_{2 s}}^{n}\left(i_{2 s+1}, i_{2 s+2}, \cdots, i_{2 p}\right) \\
\left(E^{s}\right) & \sim s ! \sum(-1)^{n} m_{i_{1} i_{2}} m_{i_{8} i_{4}} \cdots m_{i_{2 s-1} i_{s s}}^{n}\left(i_{2 s+1}, i_{2 s+2}, \cdots, i_{g, p}\right) .
\end{aligned}
$$

It is of course understood that the various irreducible parts of ( $E_{1} E_{2} \cdots E_{8}$ ) are everywhere sensed alike.*

66. The two most interesting cases are $s=p$ and $s=p-1$. In the first case we obtain, except for the sign, $\left[E_{1} E_{2} \cdots E_{p}\right]$ which is the number of zeros common to $p$ intermediary functions. In particular $\dagger$

$$
\pm\left[E^{p}\right]=p ! \sum(-1)^{n} m_{i_{1} i_{2}} m_{i_{3} i_{4}} \cdots m_{i_{2 p-1} i_{2 p}}=p ! \pi
$$

where the quantity $\pi$ is the pfaffian of the skew symmetric determinant $\left|m_{j k}\right|(j, k=1,2, \cdots, 2 p)$ of the fundamental form $F$.

For $s=p-1$ we obtain the expression for the algebraic two-cycle:

$$
\left(E^{p-1}\right) \sim(p-1) ! \sum(-1)^{n} m_{i_{1} i_{2}} m_{i_{3} i_{4}} \cdots m_{i_{2 p_{-3} i_{2 p-2}}}\left(i_{2 p-1}, i_{2 p}\right) .
$$

The coefficient of $\left(i_{2 p-1}, i_{2 p}\right)$ in the sum multiplied by $\pi$ is equal to the coeffi-

* $\left(E^{\prime}\right)$ is actually a $(2 p-2 s)$-cycle, for the functions $\phi(u-e)$ cut out hypersurfaces of $\{E\}$, and $s$ of them with the $(e)$ 's arbitrary will intersect in a $2 p-2 s$ dimensional manifold, as may be shown for example by recurrence.

† For ordinary $\theta^{\prime} \mathrm{s}$ this reduces to a classical formula due to Poincaré. 
cient $M_{i_{z p-1} i_{z p}}$ of $m_{i_{z p-1} i_{z p}}$ in the expansion of the determinant of $F$. Hence, finally

$$
\pi\left(E^{p-1}\right) \sim(p-1) ! \sum M_{\mu \nu}(\mu, \nu) \quad(\mu<\nu) .
$$

67. Since $\left(E^{p-1}\right)$ is algebraic, the corresponding periods of the integrals of the first kind of $V_{p}$ are zero, hence $\Phi=\sum M_{\mu \nu} x_{\mu} y_{\nu}$, which up to a numerical factor is the inverse of $F$, is an alternate Riemann form of $\Omega$. We shall see that it is a principal form of $\Omega$ or else is such a form changed in sign.*

Assume, indeed, first that $\left(E^{p-1}\right)$ is irreducible. We mean thereby, explicitly, that there exist $p-2$ hypersurfaces of the same algebraic system as $E$, whose intersection with $E$ is an irreducible curve. Let $\zeta$ be the contour making $\left(E^{p-1}\right)$ simply connected, and let $X=x+i y$ be one of the point coördinates on $\left(E^{p-1}\right)$. Finally, set

$$
u=u^{\prime}+i u^{\prime \prime}=\sum_{j=1}^{p} \lambda_{j} u_{j} ; \quad \xi_{\mu}+i \eta_{\mu}=\sum_{j=1}^{p} \lambda_{j} \omega_{j \mu} .
$$

We have, according to Riemann,

$$
\iint_{ \pm\left(E^{p-1}\right)}\left[\left(\frac{\partial u^{\prime}}{\partial x}\right)^{2}+\left(\frac{\partial u^{\prime}}{\partial y}\right)^{2}\right] d x d y=\int_{\zeta} u^{\prime} d u^{\prime \prime}>0 .
$$

But by means of a classical transformation of the first integral

$$
\begin{gathered}
\int_{\zeta} u^{\prime} d u^{\prime \prime}=\iint_{ \pm\left(L^{p-1}\right)} \frac{D\left(u^{\prime}, u^{\prime \prime}\right)}{D(x, y)} d x d y=\iint_{ \pm\left(K^{p-1}\right)} d u^{\prime} d u^{\prime \prime} \\
= \pm \frac{(p-1) !}{\pi} \sum M_{\mu \nu} \iint_{(\mu, \nu)} d u^{\prime} d u^{\prime \prime} \\
= \pm \frac{(p-1) !}{\pi} \sum M_{\mu \nu}\left(\xi_{\mu} \eta_{\nu}-\xi_{\nu} \eta_{\mu}\right)>0,
\end{gathered}
$$

which proves our affirmation in the case considered.

The signs \pm come from the fact that it is only for one of the sides of $\left(E^{p-1}\right)$ that the double integral will be positive.

Assume now that $E^{p-1}$ is composed of several irreducible curves $C_{1}, C_{2}$, $\cdots, C_{n}$. The cycle $\left(E^{p-1}\right)$ is composed of the sum of the cycles $\left(C_{j}\right)$ all assumed sensed in the same manner. We know that the following condition will then be fulfilled: Let $C$ be an arbitrary algebraic curve tangent to $C_{j}$ at a simple point $A$ and to $C_{h}$ at a simple point $B$, then trace in a tangent plane at $A$ and $B$, two small circuits $\zeta^{\prime}, \zeta^{\prime \prime}$ around these points. If we impose upon $\zeta^{\prime}, \zeta^{\prime \prime}$ directions corresponding to the positive circuits of $C_{j}$ and $C_{h}$, they will both be sensed alike with respect to $C$. More precisely speaking,

* Since this was written the same result has been derived in a more direct and elegant manner by Castelnuovo. See Rendiconti de i Lincei, ser. 5, vol. 30 (1921), pp. $50-55$. 
we may consider a small positive circuit $\zeta$ of $(C)$ starting from the interior of a small tube of axis $\zeta^{\prime}$ (a tube of which the transverse dimensions are very small with respect to $\zeta^{\prime}$ ), then bring it into a similar position relatively to $\zeta^{\prime \prime}$ without changing its sense. The integral $\mathcal{S}_{5} u^{\prime} d u^{\prime \prime}-\mathcal{S}_{\xi^{\prime \prime}} u^{\prime} d u^{\prime \prime}$ will be at first very small with respect to each term of the difference, and similarly when we consider $\mathcal{S}_{\zeta} u^{\prime} d u^{\prime \prime}-\mathcal{S}_{\xi^{\prime \prime}} u^{\prime} d u^{\prime \prime}$ and the final position of $\zeta$. Since, besides, the first integral does not change its sign, the two integrals $\mathcal{S}_{\xi^{\prime}} \mathcal{S}_{\xi^{\prime \prime}}^{\prime \prime}$ have the same sign. This sign will be that of every positive circuit bounding on $\left(C_{j}\right)$ or $\left(C_{h}\right)$ and we may assume that it is the sign + .

Let us draw in particular the contours $\zeta_{j}$ making the $(C)$ 's simply connected. They bound on these manifolds, hence we can sense them. If we do this in the manner just defined, we shall then have $\mathcal{S}_{\zeta_{i}} d u^{\prime} d u^{\prime \prime}>0$;

$$
\therefore \sum \int_{\zeta_{j}} u^{\prime} d u^{\prime \prime}=\sum \iint_{\left(C_{j}\right)} d u^{\prime} d u^{\prime \prime}=\iint_{ \pm\left(E^{p-1)}\right.} d u^{\prime} d u^{\prime \prime}>0 \text {, }
$$

and the rest is ended as previously. Hence finally:

THEOREM. The inverse of a non-singular fundamental form is up to the sign a principal form of $\Omega$.

Remark. If $\phi$ is of genus $p_{1}<p$, we may, as we have already stated, reduce $\Omega$ to the form

$$
\left\|\begin{array}{cc}
\Omega_{1}, & 0 \\
0, & \Omega_{2}
\end{array}\right\|
$$

where $\Omega_{1}$ is of genus $p_{1}, \phi$ being a function of the sole variables belonging to $\Omega_{1}$. $\quad F$ will then be a fundamental form belonging to $\Omega_{1}$, hence degenerate for $\Omega$, and the inverse $\Phi$ of $F$ considered as non-degenerate form of genus $p_{1}$, will be a principal form for $\Omega_{1}$, and therefore will be a degenerate alternate form of $\Omega$.

67. Before continuing with the reduction of intermediary forms, let us establish a generalization of Riemann's classical inequality. Its interest lies in the fact that it is the only one known to date for multiple integrals of the first kind.

Let $v_{1}, v_{2}, \cdots, v_{s}$ be distinct linear combinations of the variables $u_{1}, u_{2}$, $\cdots, u_{p}$, and $x_{1}, x_{2}, \cdots, x_{s}, s$ arbitrary point coördinates on $E_{1}, E_{2}, \cdots, E_{p-s}$, then set $v_{j}=v_{j}^{\prime}+i v_{j}^{\prime \prime}, x_{j}=x_{j}^{\prime}+i x_{j}^{\prime \prime}$. By starting with the relations

we find that

$$
\begin{array}{rrr}
\frac{\partial v_{j}}{\partial x_{h}}=\frac{\partial v_{j}^{\prime}}{\partial x_{h}^{\prime}}+i \frac{\partial v_{j}^{\prime \prime}}{\partial x_{h}^{\prime}}, & i \frac{\partial v_{j}}{\partial x_{h}}=\frac{\partial v_{j}^{\prime}}{\partial x_{h}^{\prime \prime}}+i \frac{\partial v_{j}^{\prime \prime}}{\partial x_{h}^{\prime \prime}}, \\
\frac{\partial \bar{v}_{j}}{\partial \bar{x}_{h}}=\frac{\partial v_{j}^{\prime}}{\partial x_{h}^{\prime}}-i \frac{\partial v_{j}^{\prime \prime}}{\partial x_{h}^{\prime}}, & -i \frac{\partial \bar{v}_{j}}{\partial \bar{x}_{h}}=\frac{\partial v_{j}^{\prime}}{\partial x_{h}^{\prime \prime}}-i \frac{\partial v_{j}^{\prime \prime}}{\partial x_{h}^{\prime \prime}}
\end{array}
$$

$$
\frac{D\left(v_{1}^{\prime}, v_{2}^{\prime}, \cdots, v_{s}^{\prime}, v_{1}^{\prime \prime}, \cdots, v_{s}^{\prime \prime}\right)}{D\left(x_{1}^{\prime}, x_{2}^{\prime}, \cdots, x_{s}^{\prime}, x_{1}^{\prime \prime}, \cdots, x_{s}^{\prime \prime}\right)}=\left(-\frac{1}{2}\right)^{\cdot}\left|\frac{D\left(v_{1}, v_{2}, \cdots, v_{s}\right)}{D\left(x_{1}, x_{2}, \cdots, x_{s}\right)}\right|^{2}
$$


and therefore

$$
\iint \cdots \int_{\left(\mathbb{E}_{1} E_{2} \cdots E_{p-\tau}\right)} d v_{1}^{\prime} d v_{2}^{\prime} \cdots d v_{s}^{\prime} d v_{1}^{\prime \prime} \cdots d v_{\bullet}^{\prime \prime} \neq 0
$$

The sign of this multiple integral depends only upon the sensing of the $(E)$ 's but not upon these hypersurfaces nor even upon $V_{p}$. Suppose in particular that $V_{p}$ possesses $p$ independent elliptic integrals which we shall still denote by $u_{1}, u_{2}, \cdots, u_{p}$ and take for hypersurfaces $E_{j}$, an hypersurface which is here algebraic, defined by

$$
u_{s+j}=\text { const. } \quad(j=1,2, \cdots, p-s) .
$$

The multiple integral is then reduced to the product by $(-1)^{\frac{s(s-1)}{s}}$ of $s$ integrals of type

$$
\iint_{\left(E_{1} E_{2} \cdots E_{p-1}\right)} d u_{1}^{\prime} d u_{1}^{\prime \prime}
$$

which have all the same sign. Hence

$$
(-1)^{\frac{s(s-1)}{2}} \iint \cdots \int_{\left(E_{1} E_{2} \cdots E_{p}\right)} d v_{1}^{\prime} d v_{2}^{\prime} \cdots d v^{\prime} d v_{1}^{\prime \prime} \cdots d v^{\prime \prime}
$$

is positive if $s$ is even and has the sign of the double integral if $s$ is odd. Let $\tau_{i_{2(p-)+1} i_{2 p-1)+2} \cdots i_{2 p}}$ be the period of the multiple integral with which we are dealing, with respect to $\left(i_{2(p-s)+1}, \cdots, i_{2 p}\right)$. The quantity

$$
(-1)^{\frac{s(s-1)}{2}} \sum(-1)^{n} m_{i_{1} i_{3}}^{1} m_{i_{3} i_{4}}^{2} \cdots m_{i_{3(p-s)-1}^{p-s} i_{3(p-))}}^{p-s} \tau_{i_{3}(p-s)+1} \cdots i_{z_{p}}
$$

is positive if $s$ is even and has the same sign as for $s=1$ if $s$ is odd. This is the generalization which we had in view.

68. It is easy to show now that we can always transform $\Omega$ into a canonical matrix $A$ in such a way that $\phi$ becomes, up to a quadratic exponential factor, a $\theta$ belonging to $A$. We can obviously assume that $\phi$ is of genus $p$.

Reduce $\Omega$ to a canonical matrix $A$ in such a way that $\Phi$ becomes the form

$$
\Phi^{\prime}=\sum_{\mu=1}^{p} e_{\mu}\left(x_{\mu} y_{p+\mu}-x_{p+\mu} y_{\mu}\right) .
$$

The transformed of $F$ is then

$$
F^{\prime}=m e_{p} \sum_{\mu=1}^{p} \frac{\left(x_{\mu} y_{p+\mu}-x_{p+\mu} y_{\mu}\right)}{e_{\mu}},
$$

where $m$ is up to the sign the greatest common divisor of the coefficients of $F$. Let $u_{1}^{\prime}, u_{2}^{\prime}, \cdots, u_{p}^{\prime}$ be the new variables and $\theta_{m}\left(u^{\prime}\right)$ a $\theta$ of order $m$ belonging to $A$ if $m$ is positive or the inverse of a $\theta$ of order $-m$ if $m$ is negative. Let 
finally $\psi\left(u^{\prime}\right)$ be the transformed of $\phi$. The meromorphic function

$$
\theta_{m}^{-1}\left(u^{\prime}\right) \cdot \phi(u)=\theta_{m}^{-1}\left(u^{\prime}\right) \psi\left(u^{\prime}\right)=\chi\left(u^{\prime}\right)
$$

behaves like an intermediary function belonging to $A$ with a fundamental form identically zero. By considering the relations for the corresponding coefficients $\alpha_{j_{\mu}}$, it is found that $\alpha_{j_{\mu}}=\alpha_{\mu j}(\mu, j \leqq p)$. Hence the product of $\chi$ by a suitable quadratic exponential behaves in a similar manner with $\alpha_{j \mu}=0(j, \mu \leqq p)$. The relations satisfied by the other $(\alpha)$ 's show that they are also zero. Hence, if $\alpha_{j \mu}^{\prime}, \alpha_{j \mu}^{\prime \prime}$ correspond to $\theta_{m}\left(u^{\prime}\right)$, and to $e^{G\left(u^{\prime}\right)} \cdot \psi$, where the exponential is the one already mentioned, we shall have $\alpha_{\mu \nu}^{\prime}=\alpha_{\mu \nu}^{\prime \prime}$ whence follows that the entire function $e^{G\left(u^{\prime}\right)} \cdot \psi$ behaves relatively to $A$ like a $\theta$-function. It is therefore a theta of order $m$, which proves our affirmation.

The integer $m$, which is the greatest common divisor of the coefficients of $F$, is also called the order of $\phi$.

The dimension of the linear system to which $\theta_{m}$ belongs, or which is the same, of the one to which $\phi$ belongs, is the pfaffian of $F^{\prime}$,

$$
\frac{m^{p} e_{p}^{p}}{\prod_{\mu}\left(e_{p} / e_{\mu}\right)}=m^{p} \cdot e_{1} e_{2} \cdots e_{p}=\pi^{\prime}
$$

Let $D$ be the unimodular transformation changing $\Omega$ into $A$. We have

$$
\Phi^{\prime}=\bar{D}^{-1} \Phi D^{-1}, \quad F^{\prime}=\bar{D} F D .
$$

Hence

$$
\pi^{\prime}=D_{\pi}
$$

Finally, if $\Delta, \Delta^{\prime}$ are the determinants formed with the real and imaginary terms of $\Omega$ and $A$, we have

$$
\Delta^{\prime}=D \Delta, \quad \therefore \pi \Delta=\pi^{\prime} \Delta^{\prime}<0 .
$$

Thus, as shown by Bagnera and de Franchis in the case $p=2$, the pfaffians of the non-degenerate fundamental forms of $\Omega$ all have the sign of $-\Delta$. By applying to $\Omega$ a suitably chosen unimodular transformation, we can arrange matters so that they will all be positive. The dimension of a complete linear system of intermediary functions will then be exactly equal to the corresponding pfaffian.

\section{$\S 4$. The relation $\rho=1+k$. Theorem of Appell-Humbert}

69 . Let $\Phi_{1}$ be a principal form, $\Phi_{2}, \Phi_{3}, \cdots, \Phi_{k+1}$ other alternate forms of $\Omega$ constituting with the first a set of $k+1$ independent forms. The conditions that $x \Phi_{1}+y \Phi_{j}$ be principal, ( $x, y$ are integers), can be expressed in the form of inequalities

$$
f_{1}(x, y)>0, \quad f_{2}(x, y)>0, \quad \cdots, \quad f_{n}(x, y)>0,
$$


where the $(f)$ 's are homogeneous polynomials with integral coefficients. These conditions, which are satisfied for $x=1, y=0$, will certainly also be when we take $y=1$, and $x$ great enough. Hence, if $\Phi_{j}$ is not principal, we can always replace it in the above system by a principal form. This will allow us to assume that all the $(\Phi)$ 's are principal forms. To $\Phi_{j}$ correspond non-degenerate intermediary functions, hence a fundamental form $F_{j}$, of genus $p$, inverse of $\Phi_{j}$. Let us represent any alternate form $G$ of genus $p$ by the point $(G)$ of a space $S_{p(2 p-1)-1}$ having its coefficients for homogeneous coördinates. The relation between a form and its inverse establishes an involutory birational correspondence between the points of this space, and if $G$ is not degenerate, the point $(G)$ is not fundamental for that correspondence. Let $\Phi, F$ be a principal form and the corresponding fundamental form. The $S_{k}$ which contains the points $(\Phi)$ is transformed by the involution into an algebraic $k$-dimensional variety $W_{k}$ containing all the $(F)$ 's. I say that there is no $W_{h}, h<k$, containing all these last points. For otherwise there would be a variety $W_{h}^{\prime}$ containing all the $(\Phi)$ 's, hence all the points $\alpha \Phi+\beta \Phi_{j}$, where $\alpha, \beta$ are arbitrary positive integers. In the neighborhood of $(\Phi)$ there would then be found within $W_{h}^{\prime}$ a point-set, everywhere dense, not contained in a space of less than $k$ dimensions, which would have as a consequence effectively $h \equiv k$.

It follows that the points $(F)$ are not contained in a space of less than $h$ dimensions. Besides, if $F, F^{\prime}$ are two fundamental forms, $\alpha F+\beta F^{\prime}$ is another fundamental form ( $\alpha, \beta$, arbitrary positive integers). Hence if $W_{k}$ were not an $S_{k}$, it would have to contain an infinity of straight lines passing through an ordinary point not situated within an $S_{k}$ which is impossible. It follows that $W_{k}$ is merely an $S_{k}$.

Let finally $F^{\prime}$ be a degenerate fundamental form of genus $p^{\prime}<p$. There corresponds to it an incermediary function in $p^{\prime}$ variables. We know that there exists then at least one intermediary function $\phi^{\prime \prime}$, of genus $p^{\prime \prime}=p-p^{\prime}$, such that ${\phi^{\prime a}}^{\prime \prime \beta}$ be of genus $p$, where $\alpha, \beta$ are arbitrary positive integers. If $F^{\prime \prime}$ is the fundamental form corresponding to $\phi^{\prime \prime},\left(\alpha F^{\prime}+\beta F^{\prime \prime}\right)$ will be in the space $S_{k}$ containing the points which correspond to the non-degenerate forms. Hence $\left(F^{\prime}\right)$ will also belong to that $S_{k}$.

Thus the fundamental forms like the Riemann forms give rise to a linear system $\infty^{k}$.

The modulus generated by the fundamental forms will have a minimum base composed of $k+1$ forms $F_{1}, F_{2}, \cdots, F_{k+1}$. Let $F$ be a fundamental form of genus $p$ corresponding to intermediary functions of order one. The coefficients of $F$ will then be prime to each other, and there exists a minimum base for fundamental forms of which $F$ is an element. Assume then $F=F_{1}$. The form $F_{j}^{\prime}=F_{j}+x F_{1}$ ( $x$ a positive integer) is not degenerate for $x$ 
arbitrary, even if $F_{j}$ is. If $F_{j}$ is degenerate, we can then replace it by $F_{j}^{\prime}$, and we thus see that there exists a minimum base for fundamental forms composed of non-degenerate forms. We shall assume that the above minimum base already has this property.

70. Let us prove now the following important

Theorem: The non-degenerate intermediary functions of a complete continuous system of such functions cut out a complete continuous system of hypersurfaces.

Let us reduce $\Omega$ to a canonical matrix $A$ in such a manner that the intermediary functions in question become $(\theta)$ 's belonging to $A$. We have then to prove that the $(\theta)$ 's of a given order $m$ cut out a complete continuous system. Let us represent $V_{p}$, as before, by equations

$$
\tau x_{j}=\theta_{n}^{j}\left[\begin{array}{l}
g \\
h
\end{array}\right](u)
$$$$
(j=1,2, \cdots, s)
$$

where $s-1$ is the dimension of the $(\theta)$ 's of order $n$ of given characteristic. We assume that this representation furnishes a non-singular $V_{p}$ in point to point correspondence without exception with $U_{2 p}$.

Let $\left\{C_{m}\right\}$ be the complete system of hypersurfaces of $V_{p}$ containing as total hypersurfaces those cut out by the $\theta_{m}$. We have to show that an arbitrary $C_{m}$ can be cut out by a $\theta_{m}$.

Let us designate by $|H|$ the system of hyperplane sections. Since $V_{p}$ is without singularities, an arbitrary hypersurface $l H$ of the complete system $|l H|$, ( $l$ great enough), can be cut out in the $S_{s-1}$ containing $V_{p}$ by a polynomial of order $l .^{*}$

If we substitute in this polynomial the $(\theta)$ 's in place of the $(x)$ 's, we find that every hypersurface of $l H$ can be cut out by a $\theta_{n l}$. But by means of an ordinary transformation of $V_{p}$, every linear system of $\left\{C_{n l}\right\}$ can be transformed into another containing an arbitrarily assigned linear system, since these systems are all representable by the points of a Picard variety of period matrix isomorphic to $\Omega$, and which is the image of an involution on $V_{p}$. It follows that all the linear systems in question have the same dimension, equal to that of a $\theta_{n l}$ of given characteristic. This proves the proposition for $\left\{C_{n l}\right\}$, when $l$ is above a certain limit.

We conclude from this that every $r C_{m}$, ( $r$ a sufficiently high multiple of $n$ ), is cut out by a $\theta_{m r}$. In the neighborhood of an arbitrary point $\left(u^{0}\right)$ of $C_{m}$ we shall have

$$
\theta_{m r}(u)=\left[f\left(u_{1}, u_{2}, \cdots, u_{p}\right)\right]^{r} \cdot g\left(u_{1}, u_{2}, \cdots, u_{p}\right),
$$

where $f$ is a polynomial in $u_{p}-u_{p}^{0}$ whose coefficients are holomorphic in $u_{1}, u_{2}, \cdots, u_{p-1}$, in the neighborhood of the set of values $u_{1}^{0}, u_{2}^{0}, \cdots, u_{p}^{0}$, and $g$ is holomorphic in the neighborhood of $\left(u^{0}\right)$, with $g\left(u_{1}^{0}, u_{2}^{0}, \cdots, u_{p}^{0}\right) \neq 0$.

•* Severi, Rendiconti di Palermo, vol. 28 (1909), pp. 33-87. 
Hence $\theta_{m r}^{1 / r}$ is holomorphic in the vicinity of $\left(u^{0}\right)$ and therefore it is an entire function. Besides, it behaves like a $\theta_{m}$ with respect to the matrix $A$, hence up to an exponential quadratic factor. perhaps, it is a $\theta_{m}$ which cuts out $C_{m}$ and the theorem is proved.

71. The preceding theorem will lead us rapidly to the value of $\rho$. Returning to the minimum base $F_{1}, F_{2}, \ldots, F_{k+1}$, for the fundamental forms, let $\phi_{i}$ be the non-degenerate intermediary function corresponding to $F_{i}$, and $C_{i}$ the hypersurface which it cuts out. I say that we cannot have a relation such as $\sum \lambda_{i} C_{i}=0$. Indeed, let $\lambda_{i}=\lambda_{i}^{\prime}-\lambda_{i}^{\prime \prime}$ where the $\left(\lambda^{\prime}\right)$ 's and the ( $\left(\lambda^{\prime \prime}\right)$ 's all are positive integers. By definition, there exists a hypersurface $E$ such that $D^{\prime}=E+\sum \lambda_{i}^{\prime} \cdot C_{i}$ and $D^{\prime \prime}=E+\sum \lambda_{i}^{\prime \prime} C_{i}$ are total hypersurfaces of the same continuous system. $E$ can be replaced by any hypersurface of a continuous system which contains it. Hence, we can always consider it as being cut out by an intermediary function $\phi$ of order $m$, high enough, belonging to a fundamental form $m F$ of genus $p$. For a suitable choice of $m$, the fundamental forms $m F+\sum \lambda_{i}^{\prime} F_{i}, m F+\sum \lambda_{i}^{\prime \prime} F_{i}$ are not degenerate, and the intermediary functions $\phi \Pi_{i} \phi_{i}^{\lambda_{i}^{\prime}}, \phi \Pi_{i} \phi_{i}^{\lambda_{i}^{\prime \prime}}$ will not be so either. Since the hypersurfaces $D^{\prime}, D^{\prime \prime}$ which they cut out belong to the same continuous system, this must also be true for the functions in question and their fundamental forms must coincide - that is, we must have

$$
\sum \lambda_{i} F_{i}=0 \text {. }
$$

Hence $\lambda_{1}=\lambda_{2}=\cdots=\lambda_{k+1}=0$, and the $(C)$ 's are effectively independent. An immediate corollary is that we must have $\rho \equiv k+1$, and since

$$
\rho \leqq k+1 \text {, }
$$

it follows that $k+1$ and $\rho$ are equal, a result whose importance is obvious. Incidentally, we have shown that the $(C)$ 's form a base for the hypersurfaces of $V_{p}$.

72. Theorem of APPELL-Humbert: Every hypersurface of $V_{p}$ may be cut out by an intermediary function.*

Let $C$ be an arbitrary hypersurface of $V_{p}$. We have $\lambda C+\sum \lambda_{i}^{\prime} C_{i}=\sum \lambda_{i}^{\prime \prime} C_{i}$ $\left(\lambda, \lambda_{i}^{\prime}, \lambda_{i}^{\prime \prime}\right.$, positive integers). Hence as before there are two hypersurfaces $D^{\prime}, D^{\prime \prime}$ cut out by non-degenerate intermediary functions, and such that $\lambda C+D^{\prime}$ and $i^{\prime \prime}$ belong totally to the same complete continuous system. Hence there are two intermediary functions $\phi, \phi^{\prime}$ cutting out $\lambda C+D^{\prime}$ and $D^{\prime}$. The function $\psi=\phi / \phi^{\prime}$ behaves like an intermediary function relatively to the periods. Besides $D^{\prime}$ may be taken arbitrarily within its continuous system. Let $D_{1}^{\prime}$ be another hypersurface of that system. There exist inter-

* See Humbert, Journal de mathématiques pures et appliquées, ser. 4 , vol. 9 (1893), pp. 29-170, for the case $p=2$. 
mediary functions $\phi_{1}, \phi_{1}^{\prime}$ cutting out $\lambda C+D_{1}^{\prime}$ and $D_{1}^{\prime}$ and we see at once that $\left(\phi \cdot \phi_{1}^{\prime}\right) /\left(\phi^{\prime} \cdot \phi_{1}\right)$ is neither zero nor infinite on any hypersurface of $V_{p}$. Hence $\psi_{1}=\phi_{1} / \phi_{1}^{\prime}=e^{G(u)} \cdot \psi$, where $G$ is an entire function. Since we may always so choose $D_{1}^{\prime}$ that $\psi_{1}$ be holomorphic at a given point $\left(u^{0}\right), \psi$ is an entire function-therefore it is an intermediary function. Thus $\lambda C$ is cut out by an intermediary function $\psi$. We may now show as in No. 70 that $\psi^{1 / \lambda}$ is an intermediary function cutting out $C$, even if $\psi$ is degenerate, which proves the theorem.

73. Let $F$ and $C$ be respectively the fundamental form and the hypersurface corresponding to $\psi$. We have $F+\sum \lambda_{i}^{\prime} F_{i}=\sum \lambda_{i}^{\prime \prime} F_{i}\left(\lambda_{i}^{\prime}, \lambda_{i}^{\prime \prime}\right.$, positive integers). Just as before there exists here a non-degenerate fundamental form $F^{\prime}$, such that $F^{\prime}+F+\sum \lambda_{i}^{\prime} F_{i}=F^{\prime}+\sum \lambda_{i}^{\prime \prime} F_{i}$ is not degenerate either. Let $\phi$ be an intermediary function belonging to $F^{\prime}$. The intermediary functions $\psi \phi \Pi_{i} \phi_{i}^{\lambda_{i}^{\prime}}, \phi \Pi_{i} \phi_{i}^{\lambda_{i}^{\prime \prime}}$ belong to the same continuous system of such functions, which leads at once to the relation $C=\sum\left(\lambda_{i}^{\prime \prime}-\lambda_{i}^{\prime}\right) C_{i}$. Thus $C_{1}, C_{2}$, $\cdots, C_{k+1}$ form a minimum base. Incidentally, these hypersurfaces are independent, hence $\sigma=1$, as we have already found before in a different manner.

74. Remarks. I. When $p=3$, we can arrive much more rapidly at the relation $\rho=1+k$. Indeed, we shall show below independently from this relation, that the arithmetic genus of an Abelian variety of genus $p$ is equal to $(-1)^{p-1}$, hence when $p$ is odd it is equal to the geometric genus. It follows in particular that $V_{3}$ is completely regular, in which case (No. 36) every two-cycle with zero periods for the integrals of the first kind, is algebraic, which gives at once $\rho=1+k$.

II. Whatever $\Omega$, the $p$-uple integral of the first kind of $V_{p}$ has at least one non-zero period since $\Omega$ is an array of $\operatorname{rank} p$. We can then apply the theorem of No. 36: Every algebraic surface which is a complete intersection in $V_{p}$ of hypersurfaces belonging to a sufficiently general linear system has for bases the traces of the bases of $V_{p}$.

III. Let us mention explicitly that, whatever $p$,

$$
\rho_{0}^{2}=\rho_{0}=R_{2}-\rho=R_{2}-(1+k)=p(2 p-1)-1-k .
$$

\section{$\S 5$. Formula for the arithmetic genera}

75. In a paper in the A.n $\mathrm{n}$ al $\mathrm{s}$ of $\mathrm{M}$ a the $\mathrm{m}$ a ti c s of 1916 the author has established this result: If $C=\sum_{i=1}^{i} \lambda_{i} C_{i}$, the arithmetic genus of $C$ is given by

$$
[C]=\left[\prod_{i=1}^{\infty}\left(1+C_{i}\right)^{\lambda_{i}}-1\right]-\left(\begin{array}{c}
\sum \lambda_{i}-1 \\
p-1
\end{array}\right) .
$$

where at the right one must expand in series, then replace the non-numerical 
tèrms of degree $\leqq p$ by the corresponding arithmetic genera. He has besides established a formula according to which $\left[(l C)^{h}\right]$ is a polynomial in $l$ with coefficients of the first degree in $\left[C^{i}\right]$.

Assume now that $C$ is a hypersurface cut out by an intermediary function of non-degenerate form $F$. If $C$ is generic in its continuous system, $V_{p}$ may be birationally transformed into a variety $V_{p}^{\prime}$, non-singular and having for hyperplane sections the complete system $|l C|$ ( $l$ sufficiently great). The dimension of the containing space is $\pi l^{p}-1$, where $\pi$ is the pfaffian of $F$. On the other hand, the postulation of $V_{p}^{\prime}$ with respect to the varieties of order $n$ of its space is $\pi(l s)^{p}$. Hence (Severi, loc. cit.),

$$
\begin{array}{r}
\pi(l s)^{p}=\left(\begin{array}{c}
s+p \\
p
\end{array}\right)\left[(l C)^{p}\right]-\left(\begin{array}{c}
s+p-1 \\
p-1
\end{array}\right)\left(\left[(l C)^{p}\right]+\left[(l C)^{p-1}\right]-1\right) \\
+\left(\left[(l C)^{p-1}\right]+\left[(l C)^{p-2}\right]\right)\left(\begin{array}{c}
s+p-2 \\
p-2
\end{array}\right) \\
+\cdots+(-1)^{p}\left([(l C)]+\left[V_{p}\right]\right) .
\end{array}
$$

If in these expressions the quantities $\left[(l C)^{h}\right]$ are replaced by their expressions in terms of genera $\left[\mathrm{C}^{i}\right]$ we obtain an algebraic equation verified for an infinite number of sets of values $(s, l)$ which cannot be thus related. The relation obtained must therefore be an identity. Making $l=1$, we have for every value of $s$

$$
\begin{aligned}
\pi s^{p}=\left[\begin{array}{c}
s+p \\
p
\end{array}\right) C^{p}-\left(C^{p}+C^{p-1}-1\right)\left(\begin{array}{c}
s+p-1 \\
p-1
\end{array}\right) \\
\left.+\cdots+(-1)^{p}\left(C+V_{p}\right)\right] .
\end{aligned}
$$

Moreover we can always find constants $t_{i}$ such that, whatever $s$,

$$
s^{p}=\sum_{i=0}^{p} t_{i}\left(\begin{array}{c}
s+i \\
i
\end{array}\right) \text {. }
$$

By comparing the two values of $s^{p}$ it is found that

$$
[C]=(-1)^{p-1}\left[\pi\left(t_{1}+t_{2}+\cdots+t_{p}\right)-1\right] .
$$

Making successively $s=0$ and $s=-1$ in the preceding formula, we obtain $t_{0}+t_{1}+\cdots+t_{p}=0, t_{0}=(-1)^{p}$, whence ultimately $[C]=\pi+(-1)^{p}$. Finally, substituting $s=0$ in the formula for $\pi s^{p}$, we find $\left[V_{p}\right]=(-1)^{p-1}$. Thus the arithmetic genus of an Abelian variety of rank one and genus $p$ is equal to $(-1)^{p-1}$. This is in agreement with the value -1 already known for the arithmetic genus of hyperelliptic surfaces.

It is interesting to observe that a certain formula predicted by Severi but for which no proof is known as yet, is verified in the case of Abelian varieties. 
Designating as before by $i_{s}$ the number of $s$-uple integrals of the first kind, we must have according to this formula

$$
\left[V_{p}\right]=i_{p}-i_{p-1}+\cdots+(-1)^{p-1} i_{1} .
$$

Since $i_{s}=\left(\begin{array}{l}p \\ b\end{array}\right)$ and

$$
1-\left(\begin{array}{l}
p \\
1
\end{array}\right)+\left(\begin{array}{l}
p \\
2
\end{array}\right)-\cdots+(-1)^{p-1} \cdot p=(1-1)^{p}-(-1)^{p}=(-1)^{p-1},
$$

the formula holds here.

76. Let $F_{i}$ be the fundamental form of $C_{i}$. The pfaffian $\pi$ will be a polynomial in $\lambda_{1}, \lambda_{2}, \cdots$, whose coefficients are simultaneous invariants of the forms $F_{i}$. Since

$$
\pi+(-1)^{p}=\left[\prod_{i}\left(1+C_{i}\right)^{\lambda_{s}}-1\right]-\left(\begin{array}{c}
\sum \lambda_{i}-1 \\
p-1
\end{array}\right),
$$

whatever the positive integers $\lambda_{i}$, this relation is an identity and the toefficients of the powers of the $(\lambda)$ 's must be equal on both sides. Comparing those of $\lambda_{1}, \lambda_{2}, \cdots, \lambda_{8}$ we obtain $\left[C_{1} C_{2} \cdots C_{8}\right]=+G_{8}$, where $G_{8}$ is a simultaneous invariant of the fundamental forms, $F_{1}, F_{2}, \cdots, F_{8}$ of which it is not necessary to give here the expression, which is easy enough to obtain. This formula solves the problem of the determination of the arithmetic genus for the complete intersections in $V_{p}$.

We verify in particular that

$$
\begin{aligned}
& {\left[C^{p}\right] }=\pi \cdot p !, \quad \text { (formula of No. 66; Poincaré) } \\
& {\left[C^{p-1}\right]=1+\frac{1}{2} \pi(p-1) \cdot p ! } \\
& {\left[C^{p-2}\right]=-1+\frac{\pi(p-2)(3 p-5)}{4 !} \cdot p !, \quad \text { etc. } }
\end{aligned}
$$

For $p=2,\left[C^{2}\right],[C],\left[C_{1} C_{2}\right]$ have precisely the values already obtained by Bagnera and de Franchis.

\section{Chapter II. Abelian Varieties with Complex Multiplication}

\section{$\S 1$. Generalities}

77. In the first chapter we have established the identity between the numbers $\rho$ and $1+k$. The next problem of interest is that of the determination of the numbers $h, k$ for a wide range of cases. The varieties with complex multiplication will furnish them readily.

In order that the transformation $T$

$$
u_{j}^{\prime}=\sum_{k=1}^{p} \lambda_{j k} u_{k} \quad(j=1,2, \cdots, p),
$$


define an algebraic correspondence on the Abelian variety of rank one $V_{p}$, belonging to the matrix $\Omega$, it is necessary and sufficient (Humbert, Scorza) that there exist relations

$$
\begin{aligned}
\sum_{k} \lambda_{j k} \omega_{k_{\mu}}=\sum_{\nu=1}^{2 p} b_{\nu \mu} \omega_{j, \nu} \\
\quad(j=1,2, \cdots, p ; \mu=1,2, \cdots, 2 p),
\end{aligned}
$$

where the $(b)$ 's are rational numbers. These relations can also be written symbolically:

$$
\left\|\lambda_{j k}\right\| \cdot \Omega=\Omega \cdot\left\|b_{\mu \nu}\right\| .
$$

If the so-called "multipliers" as defined below are rational and equal, $T$ is said to be an ordinary multiplication of $V_{p}$, while in the contrary case it is said to be a complex multiplication of the variety. If the determinant $\left|\lambda_{j k}\right|=0$ we have to deal with a complex multiplication of an Abelian variety of genus $<p$ contained in $V_{p}$. We shall assume then in general that this determinant is not zero. Besides, (Frobenius) $\left|\lambda_{j k}\right| \cdot\left|\bar{\lambda}_{j k}\right|=\left|b_{\nu \mu}\right|$, and the rank of the determinant at the right is double that of $\left|\lambda_{j k}\right|$. Hence, if one is not zero, neither is the other. Moreover, the determinant of the (b)'s is necessarily positive. Let us assume that the $(b)$ 's are integers. The determinant is then equal to a certain integer $m$ and we have to deal with a correspondence $(1-m)$. In order that we have a birational transformation, it is necessary and sufficient that the determinant be equal to one.

The multipliers of $T$ already alluded to are simply the roots of the equation in $\alpha$,

$$
\left|\lambda_{j k}-\epsilon_{j k} \cdot \alpha\right|=0 \quad\left(\epsilon_{i j}=1, \epsilon_{j k}=0 \text { if } j \neq k\right),
$$

If none of them are zero, we have a multiplication of functions of genus $p$ or non-singular multiplication.

According to Frobenius,

$$
\left|\lambda_{j k}-\epsilon_{j k} \cdot \alpha\right| \cdot\left|\bar{\lambda}_{j k}-\epsilon_{j k} \cdot \alpha\right|=\left|b_{\nu \mu}-\epsilon_{\nu \mu} \cdot \alpha\right|=F(\alpha),
$$

hence the multipliers are roots of an equation of degree $2 p$, with rational coefficients, $F(\alpha)=0$, called the characteristic equation of the complex multiplication $T$. A real root must annul each factor on the left side above and is therefore double for $F(\alpha)$. On the contrary, if a multiplier is imaginary its conjugate annuls the second factor on the left and the two may be simple roots of $F(\alpha)$. When the multiplication is singular, $F(\alpha)$ has zero roots and the determinant $\left|b_{v \mu}\right|=0$. The characteristic equation whose properties from the point of view that interests us here, have been thoroughly studied by Frobenius, will play a fundamental part in the sequence. The problem which we propose to consider is the following: Given a certain equation with rational 
coefficients, to find the most general Abelian varieties possessing a complex multiplication of which this equation is the characteristic equation, and to determine their invariants.

When the equation is arbitrary, the problem is far from simple, especially since a given Abelian variety may possess multiplications of widely different types. Now we have seen at the beginning of Part II that the problem of the determination of the numbers $h, k$, and therefore also of $\rho$, reduces itself to the following two problems: (a) To find the invariants of a pure matrix; (b) To find the simultaneous index of two pure matrices. We shall see that every complex multiplication belonging to a pure matrix has a characteristic equation of type $[f(\alpha)]^{r}=0,(f(\alpha)$ irreducible), and it is the investigation of extensive cases where the characteristic equation is of this type that will occupy us very largely in the sequence.

78. We recall here, first, the point of view and method which Scorza followed in introducing the index $h$. Let $T^{\prime}$ be another complex multiplication than $T$ and let

$$
\begin{aligned}
u_{j}^{\prime}=\sum_{k=1}^{p} \lambda_{j k}^{\prime} u_{k}, \quad \sum_{k=1}^{p} \lambda_{j k}^{\prime} \omega_{k \mu}=\sum_{\nu=1}^{2 p} b_{\nu \mu}^{\prime} \omega_{j \nu} \\
(j=1,2, \cdots, p ; \mu=1,2, \cdots, 2 p)
\end{aligned}
$$

be its equations. Whatever the rational numbers $m, m^{\prime}$, the equations $u_{j}^{\prime}=\sum_{k}\left(m \lambda_{j k}+m^{\prime} \lambda_{j k}^{\prime}\right) u_{k}$ define a new multiplication, since the relations $\sum_{k}\left(m \lambda_{j k}+m^{\prime} \lambda_{j k}^{\prime}\right) \omega_{k_{\mu}}=\sum_{\nu}\left(m b_{\nu \mu}+m^{\prime} b_{\nu \mu}^{\prime}\right) \omega_{j_{\nu}}$ are verified. This new multiplication will be designated by $m T+m^{\prime} T^{\prime}$.

Since the rank of the determinant $\left|b_{\nu \mu}\right|$ is equal to the sum of the ranks of the determinants $\left|\lambda_{j k}\right|,\left|\bar{\lambda}_{j k}\right|$, the (b)'s cannot all be zero without this being the case for all the $(\lambda)$ 's and conversely. If the terms of a multiplication are all zero we shall write $T=0$. It is clear that, given $4 p^{2}$ multiplications, we can find a linear combination of them identically zero, hence multiplications form a finite modulus, and in fact the base number is $1+h$. - This fundamental point is proved by Scorza as follows: Consider the elements of any row of $\Omega$ as homogeneous point coördinates in an $S_{2 p-1}$. To the $p$ rows correspond $p$ points which define an $S_{p-1}$. The equations (2) show that the projectivity with rational terms $B=\left\|b_{\nu \mu}\right\|$, of $S_{2 p-1}$, transforms the $S_{p-1}$ into itself. Conversely a projectivity with rational terms having this property defines a multiplication of $V_{p} . \quad B$ is said to be a Riemann projectivity of $\Omega$. If $B^{\prime}$ corresponds thus to $T^{\prime}$, to the multiplications $m T+m^{\prime} T^{\prime}$ and $T T^{\prime}$ correspond the respective Riemann projectivities $m B+m^{\prime} B^{\prime}$ and $B B^{\prime}$. Hence in particular the base numbers for the projectivities and for the multiplications are the same. Now a bilinear form of $\Omega$ defines a reciprocity of $S_{2 p-1}$ with rational terms, or "a rational reciprocity," which transforms each point of the above $S_{p-1}$ into an $S_{2 p-2}$ passing through that $S_{p-1}$. Let $C, D$, be two such 
reciprocities; $C^{-1} D$ is a Riemann projectivity of $\Omega$ and defines therefore a multiplication. Conversely to the Riemann projectivity $B$ corresponds the rational reciprocity $B C$, from which follows readily that there are exactly $1+h$ distinct multiplications of which no linear combination is a zero multiplication. A very simple discussion shows that $1+h \leqq 2 p^{2}, 1+k \leqq p^{2}$, and that if $\Omega$ is pure, $1+h \leqq 2 p, 1+k \leqq 2 p-1$. We see that if $k>0$ there is at least one complex multiplication.

We recall again that every Riemann matrix is isomorphic to one of the type

$$
\left|\begin{array}{cccc}
\omega_{1}, & 0, & \cdots, & 0 \\
0, & \omega_{2}, & \cdots, & 0 \\
0, & \cdot & \cdots \cdot & \cdot \\
0, & \cdots, & \omega_{n}
\end{array}\right|
$$

where the $(\omega)$ 's are pure Riemann matrices not isomorphic to each other or else are of the type

$$
\left\|\begin{array}{cccc}
\omega, & 0, & \cdots, & 0 \\
0, & \omega, & \cdots, & 0 \\
\cdot & \cdot & \cdot & \cdot \\
0 . & \cdot & \cdots, & \cdot
\end{array}\right\|
$$

where $\omega$ is pure. Of course we assume that two submatrices $\omega_{i}, \omega_{j}$, which enter into the type (I) are not composed with isomorphic pure matrices $\omega$ in case they are impure.

By considerations such as the above, Scorza has shown that if two Riemann matrices possess a simultaneous form, they possess isomorphic submatrices, these conditions being moreover necessary. The matrices $\omega_{i}$ in (I) do not possess therefore any simultaneous forms and if $h_{i}, k_{i}$ are their invariants we have for $\Omega$, if of type (I),

$$
1+h=\sum\left(1+h_{i}\right), \quad 1+k=\sum\left(1+k_{i}\right),
$$

while for a matrix of type (II), with $n$ terms in the main diagonal,

$$
1+h=n^{2}\left(1+h^{\prime}\right), \quad 1+k=\frac{n(n-1)}{2}\left(1+h^{\prime}\right)+n\left(1+k^{\prime}\right),
$$

where $h^{\prime}, k^{\prime}$ are the invariants of $\omega$. Hence, to find $h, k$, or $\rho$, it is sufficient to reduce $\Omega$ to the type (I) and to find the invariants $h, k$ of certain pure matrices.

By considering the representative spaces, we see at once that:

(a) Every Riemann projectivity operates separately on the submatrices $\omega_{i}$. This is an immediate consequence of the fact that the reduction to the type (I) is unique (Scorza).

(b) The characteristic polynomial $F(\alpha)$ corresponding to a Riemann pro- 
jectivity is a product $f_{1}, f_{2}, \cdots, f_{n}$, where $f_{i}$ is the characteristic polynomial of the projectivity corresponding to $\omega_{i}$.

79. Let $B$ be a Riemann projectivity of $\Omega$. Its various powers, $B^{0}=1$, $B, \cdots, B^{m}, \cdots$ are also such projectivities. The degree of an equation $f(B)=0$, ( $f$ a polynomial with integral coefficients) satisfied by these powers has a minimum $q$, and there exists a unique equation of this degree satisfied by $B$-it is the minimum equation of Frobenius.*

The integer $q$ will be called the degree of $B$.

The equations $F(\alpha)=0, f(\alpha)=0$, have the same roots, hence if $f(0)=0, B$ is singular, and conversely. When the roots of $f(\alpha)=0$ are all simple, $B$ is said to be a general projectivity. In the contrary case, it is said to be special. A projectivity is general or special according as the space of minimum dimension containing its stationary points is or is not of dimensionality $2 p-1$. We shall see below that if $B$ is special $\Omega$ is impure. For the present, let us show that if $B$ is general, non-singular, with irreducible minimum equation, its degree $q$ divides $1+h$. For, first, there exists a matrix $C$ such that $C^{-1} B C$ is a matrix with terms on one side of the principal diagonal all equal to zero, those of the diagonal itself being the roots of $F(\alpha)$ each taken with its multiplicity. From this it follows readily that the roots of the characteristic equation of $\psi(B)$ ( $\psi$ a polynomial with rational coefficients) are the numbers $\psi\left(\alpha_{i}\right)$, where $\alpha_{i}$ is any root of $F(\alpha)$. Hence in the first place, if $\psi$ is of degree $<q, \psi(B)$ is not singular.

Let now $A$ be a projectivity independent of the powers of $B$. I say that the projectivities $1, B, B, \cdots, B^{q-1}, A, A B, \cdots, A B^{q-1}$, are all independent. For otherwise there would have to be a relation $\phi(B)+A \cdot \psi(B)=0$, where $\phi, \psi$ are of degree $q-1$ at most. It is easy to see that since $\psi(B) \neq 0$, there exists a projectivity $\chi(B)=\sum a_{s} B^{s}$, such that $\psi(B) \cdot \sum a_{s} B^{s}=m$, where $m$ is a non-zero integer. Indeed the computation of the coefficients $a_{s}$ is formally the same as that which presents itself when given an algebraic number $\alpha$ such that $\psi(\alpha) \neq 0$, it is proposed to put $1 /(\psi(\alpha))$ in the form $(1 / m) \sum a_{s} \alpha^{s}$, a problem which is easily solved. We would have then finally $m A=\phi(B)$, contrary to our assumption as to $A$. The $2 q$ distinct projectivities $B^{s}, A B^{s}$ are therefore effectively independent.

Let now $A^{\prime}$ be a projectivity independent of the preceding. One may show in a similar manner that the projectivities $B^{s}, A B^{s}, A^{\prime} B^{s}(s=0,1$, $\cdots, q-1)$ are independent, etc. Continuing thus there will come a time

* The term is due to Rosati, who in a note of the Torin o A t i of May, 1916, investigated this equation more particularly from the point of view of its relations with correspondences on algebraic curves. There is also a recent note by Scorza bearing on the same subject in the $R$ e ndiconti dei $\mathrm{Lincei}$ of October, 1917.-(Added in 1922:- The results of this note have since been extensively exposed by Scorza in the Palermo Rendiconti, vol. 45 (1921), pp. 1-204. Not a few contacts with this part of our work are of course to be expected). 
when we will have exhausted all the independent projectivities. As those obtained will have been grouped in sets of $q, 1+h$ is divisible by $q$. In particular, we observe that if $1+h>q$, then certainly $1+h \equiv 2 q$.

80: We have said that if $B$ is special, $\Omega$ is impure. This point is easy to prove. Let indeed $\phi(\alpha)=0$ be the equation of which the reots of $F(\alpha)$ are simple roots. $\phi(\alpha)$ is of degree $<q$, hence $\phi(B)$ is a non-zero projectivity with multipliers all zero and therefore $\Omega$, which possesses a singular projectivity, is necessarily impure. Similarly, if $F(\alpha)=F_{1}(\alpha) \cdot F_{2}(\alpha)$, where $F_{1}, F_{2}$ are irreducible and prime to each other, $\Omega$ is impure. For $F_{1}(B)$. is a Riemann projectivity of which some multipliers but not all are zero, hence it is a singular projectivity not identically zero, and $\Omega$ is impure.

We thus see that the characteristic equations of pure matrices are all of type $[f(\alpha)]^{r}=0$, where $f$ is irreducible of degree $q=2 p / r$, and $f(B)=0$ is the minimum equation of $B$, so that its degree is also $q$.

It is interesting to notice that the impure matrices reducible to the type (II) of No. 78 possess at least one general projectivity. For if $u_{1}, u_{2}, \cdots, u_{p}$ are the variables corresponding to this matrix itself, its Abelian variety possesses the cyclic birational transformation of order $n$

$$
u_{i}^{\prime}=u_{p^{\prime}+i} \quad\left(p=n p^{\prime} ; u_{p+i}=u_{p}\right) .
$$

The corresponding Riemann projectivity is cyclic, hence general.

Thus, amongst the matrices which possess a general projectivity are found those of type (II), whether pure or impure.

81. Let then $B$ be a general Riemann projectivity with a characteristic equation $[f(\alpha)]^{r}=0$, of degree $q=2 p / r$ with $f$ irreducible. By making a change of variables, equations (1), (2) can be put in the form

$$
\begin{aligned}
& u_{j}^{\prime}=\alpha_{j} u_{j} ; \\
& \alpha_{j} \omega_{j_{\mu}}=\sum_{\nu=1}^{2 p} b_{\mu \nu} \omega_{j_{\nu}} \\
& \quad(j=1,2, \cdots, p ; \mu=1,2, \cdots, 2 p) .
\end{aligned}
$$

Let $\gamma_{1}$ be an arbitrary linear cycle of $V_{p}, \tau_{j 1}$ the corresponding period of $u_{j}$. The equations (3), (4) show that $\tau_{11} \alpha_{1}, \tau_{21} \alpha_{2}, \cdots, \tau_{p 1} \alpha_{p}$ form a system of simultaneous periods of $u_{1}, u_{2}, \cdots, u_{p}$, the corresponding cycle $\gamma_{2}$ being independent of $\gamma_{1}$. Similarly the quantities $\tau_{11} \alpha_{1}^{i-1}, \cdots, \tau_{p 1} \alpha_{p}^{i-1}$ form a system of simultaneous periods belonging to a linear cycle $\gamma_{i}$ of $V_{p}$ and since $f(\alpha)$ is irreducible, the cycles $\gamma_{1}, \gamma_{2}, \cdots, \gamma_{q}$ are independent. If $r>1$ there will be a cycle $\gamma_{a+1}$ independent of the preceding with a period $\tau_{j 2}$ for $u_{j}$. We shall have similarly cycles $\gamma_{q+1}, \cdots, \gamma_{2 q}$, the period of $u_{j}$ relatively to $\gamma_{a+i}$ being $\tau_{j 2} \alpha_{j}^{i-1}$. I say that between these $2 q$ cycles, there 
exists no homology. For otherwise there would have to be a relation

$$
\frac{\tau_{j 2}}{\tau_{j 1}}=\frac{m_{0}+m_{1} \alpha_{j}+\cdots+m_{q-1} \alpha_{j}^{q-1}}{m_{0}^{\prime}+m_{1}^{\prime} \alpha_{j}+\cdots+m_{q-1}^{\prime} \alpha_{j}^{q-1}},
$$

where the $(m)$ 's and $\left(m^{\prime}\right)$ 's are integers. From this would follow a relation with integral coefficients,

$$
n \tau_{j 2}=\tau_{j 1}\left(n_{0}+n_{1} \alpha_{j}+\cdots+n_{q-1} \alpha_{j}^{q-1}\right),
$$

and therefore a homology between the cycles $\gamma_{1}, \gamma_{2}, \cdots, \gamma_{q+1}$, which is in contradiction to the assumption as to the independence of $\gamma_{q+1}$ from the $q$ other cycles. This reasoning can be continued and we shall obtain finally $2 p$ independent cycles to which will correspond a period matrix $\Omega^{\prime}$ isomorphic to $\Omega$,

$\Omega^{\prime}=\left\|\tau_{j 1}, \tau_{j 1} \alpha_{j}, \tau_{j 1} \alpha_{j}^{2}, \cdots, \tau_{j 1} \alpha_{j}^{q-1}, \tau_{j 2}, \tau_{j 2} \alpha_{j}, \cdots, \tau_{j r} \alpha_{j}^{q-1}\right\|_{(j=1,2, \cdots, p)}$, composed with the two arrays*

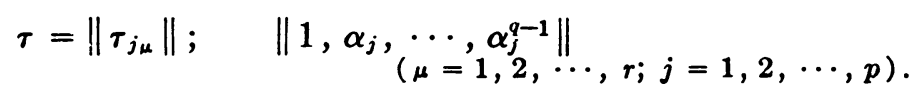

82. The projectivity $B$ being still general, assume now that $F(\alpha)=[f(\alpha)]^{r}$. $F_{1}(\alpha)$, where $f(\alpha)$ is irreducible, of degree $q$, and prime to $F_{1}(\alpha)$. Of the multipliers, $\frac{1}{2} r q$, say $\alpha_{1}, \alpha_{2}, \cdots, \alpha_{k} r q$, are roots of $f(\alpha)$. Moreover, according to a remark made above, if $g(\alpha)$ is an arbitrary polynomial with rational coefficients, there exists a complex multiplication which may be represented by $g(T)$, with multipliers $g\left(\alpha_{j}\right)(j=1,2, \cdots, p)$. There will then be rational numbers $b_{\nu \mu}$ such that

$$
\begin{aligned}
g\left(\alpha_{j}\right) \omega_{j \mu}=\sum_{\nu=1}^{p} b_{\nu \mu} \omega_{j \nu} & (j=1,2, \cdots, p ; \mu=1,2, \cdots, 2 p),
\end{aligned}
$$

and the rank of the determinant $\left|b_{\nu \mu}\right|$ will be twice the number of expressions $g\left(\alpha_{j}\right)$ that are not zero. Let us take in particular $g(\alpha)=[f(\alpha)]^{r}$. We shall have

$$
\sum_{\nu} b_{\nu \mu} \omega_{j \nu}=\underset{\left(\mu=1,2, \cdots, 2 p ; j=1,2, \cdots, \frac{1}{2} r q\right) .}{0}
$$

Hence $u_{1}, u_{2}, \cdots, u_{\xi r q}$ form a system of reducible integrals of the variety $V_{p}$ belonging to $\Omega$ and $T$ operates separately upon them. From this, we conclude that $\Omega$ is isomorphic to a matrix

$$
\left\|\begin{array}{cl}
\Omega_{1} & 0 \\
0 & \Omega_{2}
\end{array}\right\|
$$

\footnotetext{
* For $r=1, p$ arbitrary, this reduction has already been indicated by Scorza in his $\mathbf{P}$ a lermo Rendiconti paper of 1916, p. 24. For $r=p=2$ it has been repeatedly used by Bagnera and de Franchis.
} 
where $\Omega_{1}$ is of genus $\frac{1}{2} r q$ and $T$ operates separately upon it in correspondence with the characteristic equation $[f(\alpha)]^{r}=0$, and upon $\Omega_{2}$ with the equation $F_{1}(\alpha)=0 . \Omega_{1}$ is therefore isomorphic to a matrix of type (II). By reasoning on $\Omega_{2}$ as we have on $\Omega$ and continuing, we obtain finally the following result: Let $F(\alpha)=\left[f_{1}(\alpha)\right]^{r_{1}} \cdots\left[f_{n}(\alpha)\right]^{r_{n}}$ be the decomposition of $F(\alpha)$ into irreducible factors prime to each other. $\Omega$ is isomorphic to a matrix,

$$
\left\|\begin{array}{cccc}
\Omega_{1}, & 0, & \cdots, & 0 \\
0, & \Omega_{2}, & \cdots, & 0 \\
\cdot & \cdot & \cdot & \cdot \\
\cdot & \cdot & \cdots, & \Omega_{n}
\end{array}\right\|
$$

where $\Omega_{j}$ (which is of type (II)) is transformed separately by $T$ with the characteristic equation $\left[f_{j}(\alpha)\right]^{r=}=0 . \quad \Omega_{j}$ can be put in the form indicated in No. 81 .

Let $h_{j}, k_{j}, \rho_{j}$, be the invariants of $\Omega_{j}$. We have then at once (Scorza) the relations

$$
\begin{aligned}
1+h & =\sum\left(1+h_{j}\right)+2 \sum \lambda_{j k}, \\
1+k & =\sum\left(1+k_{j}\right)+\sum \lambda_{j k}, \\
\rho & =\sum \rho_{j}+\sum \lambda_{j k},
\end{aligned}
$$

where $\lambda_{j k}$ is the simultaneous index of $\Omega_{j}$ and $\Omega_{k}$. The last equation just written has already been obtained at the end of Part $I$.

It follows from our whole discussion that the determination of the invariants. of any Riemann matrix is reducible to the following two problems: (a) To determine the invariants of a matrix such as $\Omega_{j}$, that is possessing a general Riemann projectivity with characteristic equation of type $[f(\alpha)]^{r}=0$ ( $f$ irreducible). (b) To determine the simultaneous index of two such matrices.

Let us observe that if $\Omega$ is of type (II), with $T$ not operating separately upon the $(\omega)$ 's, and more especially if $\Omega$ is pure, then $n=1$, and $F(\alpha)$ is the exact power of an irreducible polynomial, whose degree $q$ is what we have called the degree of $T$. As closely related to all this we recall the two notes of Rosati and Scorza already referred to.

We recall again that if the simultaneous index of two matrices $\Omega$ and $\Omega^{\prime}$ is not zero, they are isomorphic to two matrices, (Scorza)

$$
\left\|\begin{array}{cl}
\Omega_{1}, & 0 \\
0, & \Omega_{2}
\end{array}\right\|, \quad\left\|\begin{array}{cl}
\Omega_{1}, & 0 \\
0, & \Omega_{2}^{\prime}
\end{array}\right\| .
$$

The problem which we propose to attack is the determination of $h$ and $k$ for a wide range of matrices with general projectivities, with special emphasis on pure matrices. Before we enter upon this task we shall add a last remark concerning the characteristic equation. 
83. According to Frobenius* if $T$ is a principal transformation, that is, a transformation which maintains identically invariant a principal form of $\Omega$, the multipliers have all the same absolute value, say $\beta$. Assume that $\Omega$ is pure, which requires that $F(\alpha)=[f(\alpha)]^{r}$, where $f$ is irreducible and of degree $q=2 p / r$. We shall have $\beta^{q r}=\left|b_{v \mu}\right|$. Moreover $\beta^{q}$ is a rational number, hence the determinant $\left|b_{\nu \mu}\right|$ in this case is the exact power of a rational number. A real multiplier will then be equal to $\pm \beta$. If $q$ is odd the multiplication $T^{q}$ will possess a rational number for root of its characteristic equation. As the characteristic polynomial of $T^{q}$ must still be the exact power of an irreducible polynomial, $T^{q}$ will necessarily be an ordinary multiplication and $\alpha_{j}^{q}=\beta^{q}=m$, where $m$ is a rational number. Hence, finally, $f(\alpha)=\alpha^{q}-m$. Thus the characteristic equation of a principal transformation of odd degree of a pure matrix is of the form $\left(\alpha^{q}-m\right)^{r}=0,(m$ a rational number).

84. Permutable Projectivities. According to Scorza, the transformation of coördinates

$$
x_{s}=\sum_{j=1}^{p} \omega_{j s} X_{j}+\sum_{j=1}^{p} \bar{\omega}_{j s} X_{p+j}
$$

reduces the equations of the projectivity $B$ of $S_{2 p-1}$ to the form

$$
\sigma X_{j}^{\prime}=\sum_{h=1}^{p} \lambda_{h j} X_{h} ; \quad \sigma X_{p+j}^{\prime}=\sum_{h=1}^{p} \bar{\lambda}_{h j} X_{p+h} \quad(j=1,2, \cdots, p),
$$

and since this transformation of coördinates is independent of the projectivity considered, it follows readily that if two projectivities are permutable, the same holds for the corresponding complex multiplications and conversely.

Let now $B_{1}, B_{2}, \cdots, B_{8}$ be permutable projectivities. The corresponding multiplications will also be permutable and the projectivities permutable with the $(B)$ 's will be of type $\phi\left(B_{1}, B_{2}, \cdots, B_{s}\right)$, where $\phi$ is a polynomial with rational coefficients, of degree $q_{i}-1$ at most in $B_{i}\left(q_{i}\right.$ is the degree of $\left.B_{i}\right)$. The system of projectivities $\phi\left(B_{1}, B_{2}, \cdots, B_{s}\right)$ is in every way analagous to the system of numbers of an algebraic domain. In particular, there exists a projectivity $B_{0}$, of degree $q$, such that any other is a linear combination of its powers.

Among the projectivities of the set, those with integer terms play the same part as its integers for an algebraic domain. Like these integers, they satisfy an equation whose first coefficient is unity, as follows from the fact that the roots of the minimum equation are also roots of the equation $F(\alpha)=0$ which is of this nature. These roots are therefore algebraic integers and their symmetric functions are ordinary integers. The projectivities here considered possess therefore a minimum base with $q$ terms $B_{1}, B_{2}, \cdots, B_{q}$.

* Journal fur die reine und angewandte Mathematik, vol. 95 (1883), pp. 264-297. 
A complex multiplication permutable with any other has necessarily all multipliers equal, their common value being an imaginary quadratic number $\alpha$. Replacing if necessary $\Omega$ by a suitably chosen isomorphic matrix, we may assume that $\alpha=i \sqrt{d}$ ( $d$ a positive integer). The array $\tau$ can then be reduced to one with $p$.rows and columns

$$
\left\|\begin{array}{cccc}
1, & 0, & \cdots, & 0 \\
0, & 1, & \cdots, & 0 \\
\cdot & \cdot & \cdot & \cdot \\
\cdot & \cdot & \cdots, & 1
\end{array}\right\|
$$

and $\Omega$ is a matrix with $p$ elliptic integrals, of the type called by Scorza "with maximum indices," for which $1+h=2(1+k)=2 p^{2}$.

In general assume that for some general projectivity $F(\alpha)=[f(\alpha)]^{r}$ ( $f$ irreducible and of degree $q$ ), and that among the quantities $\alpha_{1}, \alpha_{2}, \cdots, \alpha_{p}$, are found $\frac{1}{2} q$ roots each taken $r$ times, which requires that all roots of $f(\alpha)$ be imaginary. The array $\tau$ is then of the form

$$
\left\|\begin{array}{c}
\tau_{1} \\
\tau_{1} \\
\vdots \\
\cdot \\
\tau_{1}
\end{array}\right\|
$$

where $\tau_{1}$ is a square array of the same type as above with $r$ rows and columns, and $\Omega$ is impure of type (II) composed $r$ times with the matrix

$$
\left\|1, \alpha_{j}, \cdots, \alpha_{j}^{q-1}\right\| \quad \quad\left(j=1,2, \cdots, \frac{1}{2} q\right) .
$$

\section{\$2. Complex multiplication with irreducible characteristic equation.}

85. As before, let $F(\alpha)=0$ be the characteristic equation. We shall assume the roots $\alpha_{1}, \alpha_{2}, \cdots, \alpha_{2 p}$ arranged in such order that $\bar{\alpha}_{j}=\alpha_{p+j}$ $(j<p)$, and we propose to investigate the matrix

$$
\Omega=|| 1, \alpha_{j}, \cdots, \alpha_{j}^{2 p-1} \| \quad(j=1,2, \cdots, p) .
$$

In order that it be a Riemann matrix there must exist an alternate Riemann form

$$
\sum_{\mu=1}^{2 p} c_{\mu \nu} x_{\mu} y_{\nu}
$$

This requires that

$$
\sum_{\mu, \nu=1}^{2 p} c_{\mu \nu}\left(\alpha_{j}^{\mu-1} \alpha_{k}^{\nu-1}-\alpha_{j}^{\nu-1} \alpha_{k}^{\mu-1}\right)=0 \quad(j, k=1,2, \cdots, p),
$$


and that the Hermitian form

$$
\sum_{j, k=1}^{\infty} A_{j k} x_{j} \bar{x}_{k} ; \quad A_{j k}=-\frac{1}{4 i} \sum_{\mu, \nu=1}^{2 p} c_{\mu \nu}\left(\alpha_{j}^{\mu-1} \alpha_{p+k}^{\nu-1}-\alpha_{j}^{\nu-1} \alpha_{j+k}^{\mu-1}\right),
$$

be definite positive.

Let $G$ be the group of $F(\alpha)=0$. The equation (6) will be verified when we replace the pair $\left(\alpha_{j}, \alpha_{k}\right)$ by any pair of roots which may be derived from the pairs taken out of the set $\alpha_{1}, \alpha_{2}, \cdots, \alpha_{p}$ by the substitutions of $G$. On the other hand, in order that (7) be definite; it is necessary that none of the $\left(A_{i i}\right)$ 's be zero. Hence, as a necessary condition among the pairs of roots derived from those with indices both $\leqq p$ must not figure a pair of conjugate roots.

I say that this last condition is sufficient to insure that $\Omega$ be a Riemann matrix. For let $\left(\alpha_{j_{1}}, \alpha_{k_{1}}\right), \cdots,\left(\alpha_{j_{0}}, \alpha_{k_{s}}\right)$ be the $s$ pairs derived from those of indices $\leqq p,\left(\alpha_{j_{1}^{\prime}}^{\prime}, \alpha_{k_{1}^{\prime}}\right), \cdots,\left(\alpha_{j_{t}^{\prime}}, \alpha_{k_{t}^{\prime}}\right),\left(\alpha_{1}, \alpha_{p+1}\right), \cdots,\left(\alpha_{p}, \alpha_{2 p}\right)$, the $p+t$ pairs remaining with $s+p+t=p(2 p-1)$. Finally consider the equations in the unknowns $c_{\mu \nu}$ :

$$
\begin{array}{rc}
\sum_{\mu, \nu=1}^{2 p} c_{\mu \nu}\left(\alpha_{j_{n}}^{\mu-1} \alpha_{k_{n}}^{\nu-1}-\alpha_{j_{n}}^{\nu-1} \alpha_{k_{n}}^{\mu-1}\right)=0 & (n=1,2, \cdots, s), \\
\sum_{\mu, \nu=1}^{2 p} c_{\mu \nu}\left(\alpha_{j_{n}^{\mu}}^{\mu-1} \alpha_{k_{n}^{\nu}}^{\nu-1}-\alpha_{j_{n}^{\nu}}^{\nu-1} \alpha_{k_{n}^{\mu}}^{\mu-1}\right)=d_{n} & (n=1,2, \cdots, t), \\
-\frac{1}{4 i} \sum_{\mu, \nu=1}^{2 p} c_{\mu \nu}\left(\alpha_{j}^{\mu-1} \alpha_{p+j}^{\nu-1}-\alpha_{j}^{\nu-1} \alpha_{p+j}^{\mu-1}\right)=e_{j} & (j=1,2, \cdots, p) .
\end{array}
$$

The determinant of the coefficients is up to the sign

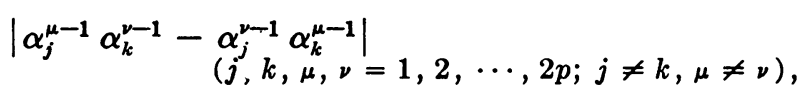

or the determinant formed with the minors of order two of the determinant of Vandermonde of $\alpha_{1}, \alpha_{2}, \cdots, \alpha_{2 p}$, hence equal to a power of that determinant and therefore different from zero. It follows that there will be a unique solution for the (c)'s. Whether these coefficients are rational or not, we can consider the corresponding Hermitian form (7). It will be definite positive if the $p$ roots of the equation in $\beta$,

$$
\left|A_{j k}-\epsilon_{j k} \beta\right|=0 \quad\left(\epsilon_{j i}=1 ; \epsilon_{j k}=0, j \neq k\right)
$$

are all positive. But when the $(d)$ 's are all zero, these $p$ roots are equal to the $(e)$ 's, since $(7)$ becomes then

$$
\sum_{j=1}^{p} e_{j} x_{j} \bar{x}_{j}
$$


Hence when we take the $(d)$ 's sufficiently small in absolute value and the $(e)$ 's positive, the roots in question will all be positive and the Hermitian form will be definite positive.

Let us consider, as we have already had occasion to do, the (c)'s as homogeneous point coördinates in an $S_{p(2 p-1)-1}$. The point whose coördinates satisfy the $s$ equations (8) is contained in a rational $S_{p(2 p-1)-s-1}$, by which we mean that the space contains $p(2 p-1)-s$ independent points with rational coördinates. We can see this at once by observing that equations (8) are simply permuted among themselves by the substitutions of $G$ and therefore have as many independent solutions in rational numbers as they have independent solutions in general. Their independence results from the fact that the array of the coefficients is composed of $s$ rows taken from a non-zero determinant.

In the $S_{p(2 p-1)-s-1}$ in question there will then be a rational point differing as little as we please from any given point and in particular from the point whose coördinates satisfy the equations (8), (9), (10), where the $(d)$ 's are sufficiently small and the (e)'s positive. In these conditions $\sum c_{\mu \nu} x_{\mu} y_{\nu}$ will be a principal form of $\Omega$, which proves our proposition.

Thus, given an irreducible equation of degree $2 p$ with roots all imaginary, in order that there correspond to it a Riemann matrix $\Omega$, it is necessary and sufficient that its group does not permute any pair of roots taken from a set of $p$ roots among which none are conjugate to each other, with a pair of conjugate roots.

When $\Omega$ exists, $1+k$ is obviously equal to the number of independent solutions of the equations (8), that is $1+k=p(2 p-1)-s=p+t$.

86. To determine $h$, we must find the number of independent rational solutions possessed by the equations in the unknowns $c_{\mu \nu}$,

$$
\sum_{\mu, \nu=1}^{2 p} c_{\mu \nu} \alpha_{j}^{\mu-1} \alpha_{k}^{\nu-1}=0 \quad(j, k=1,2, \cdots, p) .
$$

This equation must be satisfied when $\alpha_{j}, \alpha_{k}$ are permuted and also when they are replaced by any one of the pairs of roots $\left(\alpha_{j_{n}}, \alpha_{k_{n}}\right),\left(\alpha_{k_{n}}, \alpha_{j_{n}}\right)$ $(n=1,2, \cdots, s) ;\left(\alpha_{j}, \alpha_{j}\right)(j=1,2, \cdots, 2 p)$; whence the equations

$$
\begin{array}{rr}
\sum_{\mu, \nu=1}^{2 p} c_{\mu \nu} \alpha_{j_{n}}^{\mu-1} \alpha_{k_{n}}^{\nu-1}=0, \quad \sum_{\mu, \nu=1}^{2 p} c_{\mu \nu} \alpha_{k_{n}}^{\mu-1} \alpha_{j_{n}}^{\nu-1} & =0 \\
& (n=1,2, \cdots, s), \\
\sum_{\mu, \nu=1}^{2 p} c_{\mu \nu} \alpha_{j}^{\mu+\nu-2}=0, & (j=1,2, \cdots, 2 p) .
\end{array}
$$

The array of the coefficients is composed of $2(p+s)$ rows of the determinant

$$
\left|\alpha_{j}^{\mu-1} \alpha_{k}^{\nu-1}\right| \quad(\mu, \nu, j, k=1,2, \cdots, 2 p),
$$


which is not zero. The above equations are therefore independent and as they are merely permuted by the substitutions of $G$, they will possess as many independent rational solutions as independent solutions in general. Hence, finally $1+h=4 p^{2}-2 p-2 s=2(1+k)$. Thus the invariants of $\Omega$ satisfy the relations $1+h=2(1+k)=2 p(2 p-1)-2 s=2(p+t)$.

87. The preceding result gives rise to some interesting observations. For we have seen that when a matrix is pure, $1+h \leqq 2 p$. Hence, in order that $\Omega$ be pure, it is necessary that $s \geqq p(2 p-1)-p$. On the other hand, the $p$ pairs of roots $\left(\alpha_{j}, \alpha_{p+j}\right)$ are not among the pairs $\left(\alpha_{j_{n}}, \alpha_{k_{n}}\right)$ $(n=1,2, \cdots, s)$. Hence in order that $\Omega$ be pure, it is necessary that $s=p(2 p-1)-p$, which requires that the only pairs that may not be lerived from those of indices $\leqq p$ by the substitutions of $G$, are the pairs of conjugate roots.

Let $z_{1}=m_{1} \alpha_{1}+m_{2} \alpha_{2}+\cdots+m_{2 p} \alpha_{2 p}$ be a Galois function of $F(\alpha)=0$, the $(m)$ 's being arbitrary integers, and consider the Galois resolvent $\phi(z)=0$ of which $z_{1}$ is a root. The $(\alpha)$ 's are rational functions of $z_{1}$ with real coefficients. Hence $z_{1}$ is not real, as can also be seen directly. Replacing $z_{1}$ by $\bar{z}_{1}$ in these rational functions, we obtain a substitution $S$, of order two of $G$, which has the property of permuting any two conjugate roots. Moreover, if another substitution $S^{\prime}$ had the same property, we would have $S S^{\prime}=1$, hence $S^{\prime}=S^{-1}=S$ and therefore $S$ is unique.

Let us now return to $\Omega$. In order that it be a Riemann matrix and pure, it is necessary and sufficient: (a) That $S$ be permutable with all the substitutions of $G$. (b) That among the corresponding roots, $\alpha_{1}, \alpha_{2}, \cdots, \alpha_{p}$, none be conjugate, and moreover that from the pairs $\left(\alpha_{j}, \alpha_{k}\right)$ it be possible to derive all the pairs of non-conjugate roots by the substitutions of $G$. To begin with, the second condition is obviously necessary. Let us show that so is the first. For $G$ must not permute the pairs of conjugate roots with pairs of roots that are not conjugate. Hence, if $T$ is an arbitrary substitution of $G$ and if $T \alpha_{j}=\alpha_{k}$ we must have $T S \alpha_{j}=S \alpha_{k}$, whence $S^{-1} T S=T$, and $T$ is effectively permutable with $S$.

Let us show that our two conditions are also sufficient. For, if $T \alpha_{j}=\alpha_{k}$, we shall also have $T \bar{\alpha}_{j}=T S \alpha_{j}=S T \alpha_{j}=S \alpha_{k}=\bar{\alpha}_{k}$, and the pair $\left(\alpha_{j}, \bar{\alpha}_{j}\right)$ is permuted with $\left(\alpha_{k}, \bar{\alpha}_{k}\right)$; hence the pairs of conjugate roots are not permuted with any others by the substitutions of the group. The second condition insures then that $t=0$, the pairs $\left(\alpha_{j}, \bar{\alpha}_{j}\right)$ being all alone excluded. The equations for the coefficients of an alternate Riemann form are here

$$
\begin{aligned}
\sum_{\mu, \nu=1}^{2 p} c_{\mu \nu}\left(\alpha_{j}^{\mu-1} \alpha_{k}^{\nu-1}-\alpha_{j}^{\nu-1} \alpha_{k}^{\mu-1}\right) & =0 \\
(j, k & =1,2, \cdots, 2 p ; j \neq p+k) .
\end{aligned}
$$


The quantities

$$
A_{j j}=-\frac{1}{4 i} \sum_{\mu, \nu=1}^{2 p} c_{\mu \nu}\left(\alpha_{j}^{\mu-1} \alpha_{p+j}^{\nu-1}-\alpha_{j}^{\mu-1} \cdot \alpha_{p+j}^{\mu-1}\right)
$$

will not all be zero, for otherwise the (c)'s would also be zero. But if one of the quantities $A_{j j}$ is zero, all the others will also be zero for they are transitively permuted by the substitutions of $G$. Hence none of them is zero and the Hermitian form corresponding to the arbitrary Riemann form considered becomes here $\sum_{j=1}^{p} A_{j j} x_{j} \bar{x}_{j}$, and is always of genus $p$. Hence finally every Riemann form of $\Omega$ is of genus $p$ and this matrix is actually pure. This completes the proof.

Observe that the invariants of the pure matrix will be given by

$$
1+h=2(1+k)=2 p,
$$

$h$ having the maximum value which it may have for such a matrix.

88. A particularly interesting case is that where $p$ is prime. Then $\Omega$, which is of type (II), is certainly pure, whence the following result: Let $F(\alpha)=0$ be an irreducible equation of degree $2 p$ ( $p$ prime), with roots all imaginary. Either the group of the equation permutes only among themselves the pairs of conjugate roots, or else in every set of $p$ roots there are at least two which are permutable with a pair of conjugate roots.

89. Let us endeavor to characterize $F(\alpha)$ when its group is permutable with $S^{*}$ or, which is the same, when there is a corresponding pure matrix $\Omega$. The group $G$ merely permutes the equations in the unknowns $d$,

$$
\bar{\alpha}_{j}=d_{0}+d_{1} \alpha_{j}+\cdots+d_{2 p-1} \alpha_{j}^{2 p-1} \quad(j=1,2, \cdots, 2 p),
$$

and hence these equations possess a rational solution which besides is unique. This means that $\bar{\alpha}_{j}=Q\left(\alpha_{j}\right)$, where $Q$ is a polynomial with rational coefficients independent of $j$. Thus every root can be expressed as a rational function, the same for all of them, of its conjugate. Conversely if $\alpha_{j}=Q\left(\alpha_{j}\right)$, whatever $j, S$ and $G$ are permutable. For denoting still by $z_{1}$ the Galois function, we shall have first

$$
\alpha_{j}=R_{j}\left(z_{1}\right), \quad \bar{\alpha}_{j}=Q\left[R_{j}\left(z_{1}\right)\right] .
$$

We may then obtain every substitution of $G$ by replacing $z_{1}$ by a suitably chosen root $z_{2}$ of the Galois equation. If $T \alpha_{j}=\alpha_{k}$, we shall have

$$
\alpha_{k}=T \alpha_{j}=R_{j}\left(z_{2}\right), \quad T \alpha_{j}=Q\left[R_{j}\left(z_{2}\right)\right]=Q\left(\alpha_{k}\right)=\bar{\alpha}_{k},
$$

that is, $T S \alpha_{j}=S T \alpha_{j}$, or $T S=S T$.

* From this property follows already that the solution of the characteristic equation is reducible to that of an equation of degree $p$ followed by a quadratic, but this result is inoufficient for some applications which we have in view. 
Thus, in order that $S$ and $G$ be permutable, it is necessary and sufficient that whatever $j, \bar{\alpha}_{j}=Q\left(\alpha_{j}\right)$, where $Q$ is a polynomial with rational coeffcients independent of $j$.

But we can go still further. Indeed, the $p$ points of an $S_{2 p-1}$ whose nonhomogeneous point coördinates are $\alpha_{j}+\bar{\alpha}_{j}, \alpha_{j}^{2}+\bar{\alpha}_{j}^{2}, \ldots, \alpha_{j}^{2 p-1}+\bar{\alpha}_{j}^{2 p-1}$ are certainly distinct, for if two of them had their first two coördinates equal, $F(\alpha)$ would have a double root. Given arbitrary integers $d_{1}, d_{2}, \cdots, d_{2 p-1}$, the distances from these points to the corresponding hyperplane

$$
d_{1} x_{1}+d_{2} x_{2}+\cdots+d_{2 p-1} x_{2 p-1}=0
$$

are therefore all different. Whence the following conclusion: If we set

$$
\alpha_{j}^{\prime}=d_{1} \alpha_{j}+d_{2} \alpha_{j}^{2}+\cdots+d_{2 p-1} \alpha_{j}^{2 p-1},
$$

the quantities $\alpha_{j}^{\prime}+\bar{\alpha}_{j}^{\prime}$ are all distinct. In other words $\Omega$ possesses a Riemann projectivity with irreducible characteristic equation and such that if its multipliers are still denoted by $\alpha_{1}, \alpha_{2}, \cdots, \alpha_{p}$, the $p$ sums $2 \zeta_{j}=\alpha_{j}+\bar{\alpha}_{j}$ are all distinct. If we adjoin $\zeta_{j}$ to the domain of rationality, the pair of conjugate roots $\alpha_{j}, \bar{\alpha}_{j}$ is determined in one and only one way, and their product $\alpha_{j} \bar{\alpha}_{j}$ is therefore a rational function of $\zeta_{j}$. On the other hand the $(\zeta)$ 's are transitively permuted by the substitutions of $G$, hence they satisfy an irreducible equation $\psi(\zeta)=0$ of degree $p$, and the characteristic equation is of the form

$$
\prod_{j=1}^{p}\left(\alpha^{2}-2 \zeta_{j} \alpha+R\left(\zeta_{j}\right)\right)=0,
$$

where $R$ is a polynomial of degree $p-1$ with rational coefficients and such that $\zeta_{j}^{2}<R\left(\zeta_{j}\right)(j=1,2, \cdots, p)$. Of course these conditions are not sufficient to make certain that $\Omega$ is pure. For example, take the case $p=2$. The characteristic equation is of the form

$$
\left(\alpha^{2}-2 \zeta_{1} \alpha+m \zeta_{1}+n\right)\left(\alpha^{2}-2 \zeta_{2} \alpha+m \zeta_{2}+n\right)=0,
$$

where $m, n$ are rational and $\zeta_{1}, \zeta_{2}$ are the two roots of an equation

$$
a \zeta^{2}+b \zeta+c=0
$$

with coefficients $a, b, c$, integers and $a>0$. We must have $\zeta_{j}^{2}<m \zeta_{j}+n$ $(j=1,2)$, which is equivalent to the inequalities

$$
\begin{aligned}
a(a n+c)^{2}-b(a n+c)(a m+b)+c(a m+b)^{2} & >0, \\
(a m+b)\left(2 n a^{2}-m a b+2 a c-b^{2}\right) & >0 .
\end{aligned}
$$

Moreover, in order that $\Omega$ be pure, it is necessary that the pairs of conjugate roots be transitively permuted. This requires that $\alpha_{1} \alpha_{2}+\bar{\alpha}_{1} \bar{\alpha}_{2}$ be irrational, a condition which is besides sufficient. A very simple calculation shows that 
this is equivalent to requiring that

$$
a\left[a(a n+c)^{2}-b(a n+c)(a n+b)+c(a n+b)^{2}\right]
$$

be not the square of a rational number.

When these conditions are satisfied, the matrix

$$
\left\|\begin{array}{llll}
1, & \alpha_{1}, & \alpha_{1}^{2}, & \alpha_{1}^{3} \\
1, & \alpha_{2}, & \alpha_{2}^{2}, & \alpha_{2}^{3}
\end{array}\right\|
$$

is a Riemann matrix and pure.

It is scarcely possible to go further when the equation $F(\alpha)=0$ is arbitrary and so we shall pass on to the investigation of the particularly important case where it is Abelian, when the preceding results may be completed and much increased in precision. 